\title{
Synthesis of Pyrazoles Utilizing Ambiphilic Reactivity of
}

\section{Hydrazones}

Haruo Matsuzaki, Norihiko Takeda, Motohiro Yasui, Yuta Ito, Keiji Konishi, Masafumi Ueda*

Kobe pharmaceutical University, Motoyamakita, Higashinada, Kobe 658-8558, Japan

E-mail: masa-u@kobepharma-u.ac.jp

Table of contents

Page

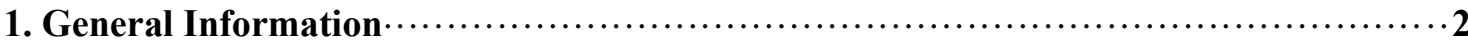

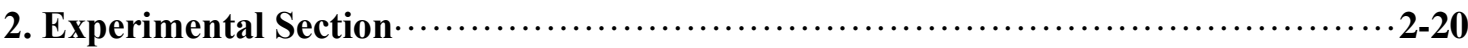

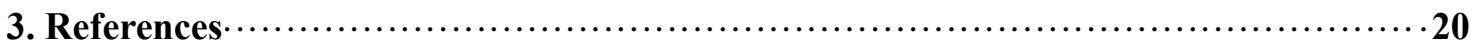

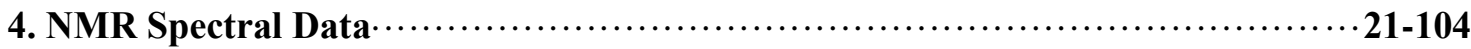




\section{General Information}

NMR spectra were recorded at $300 \mathrm{MHz} / 75 \mathrm{MHz}\left({ }^{1} \mathrm{H}\right.$ NMR $/{ }^{13} \mathrm{C}$ NMR $), 500 \mathrm{MHz} / 125 \mathrm{MHz}\left({ }^{1} \mathrm{H}\right.$ $\left.\mathrm{NMR} /{ }^{13} \mathrm{C} \mathrm{NMR}\right)$ or $600 \mathrm{MHz} / 150 \mathrm{MHz}\left({ }^{1} \mathrm{H} \mathrm{NMR} /{ }^{13} \mathrm{C}\right.$ NMR) using Varian MERCURY plus 300 (300 MHz), Varian NMR system AS 500 (500 MHz), or Bruker Avance III HD (600 MHz) spectrometers. Chemical shifts $(\delta)$ are reported as follows: chemical shift, multiplicity ( $=$ singlet, $\mathrm{d}$ $=$ doublet, $\mathrm{t}=$ triplet, $\mathrm{q}=$ quartet, $\mathrm{m}=$ multiplet), coupling constants, and integration. Infrared (IR) spectra were recorded on a Perkin-Elmer SpectrumOne A spectrometer. The high-resolution mass spectra (HRMS) were obtained using Thermo Fischer Scientific Exactive Orbitrap mass spectrometer by ESI technique. Melting points (uncorrected) were determined on BÜCHI M-565 apparatus. Preparative TLC separations (PTLC) were carried out on precoated silica gel plates (E. Merck 60F254). Methanesulfonic acid $(\mathrm{MsOH})$ was purchased from Nacalai Tesque, Inc. Hydrazones $\mathbf{1 a}-\mathbf{1} \mathbf{j}^{1 \mathrm{ad}}, \mathbf{4} \mathrm{A}^{1 \mathrm{~b}}, \mathbf{4} \mathrm{I}^{\mathrm{c}}, \mathbf{4} \mathrm{J}^{1 \mathrm{~d}}, \mathbf{4} \mathrm{L}^{1 \mathrm{e}}, \mathbf{4} \mathrm{M}^{1 \mathrm{f}}, \mathbf{4} \mathrm{O}^{1 \mathrm{~g}}, \mathbf{4} \mathrm{S}^{1 \mathrm{~d}}$ were prepared according to literatures, respectively. The spectra data of these known compounds were identical with those reported in the literatures, respectively.

\section{Experimental Section}

\subsection{Preperatioin of hydrazones (General procedure-I).}

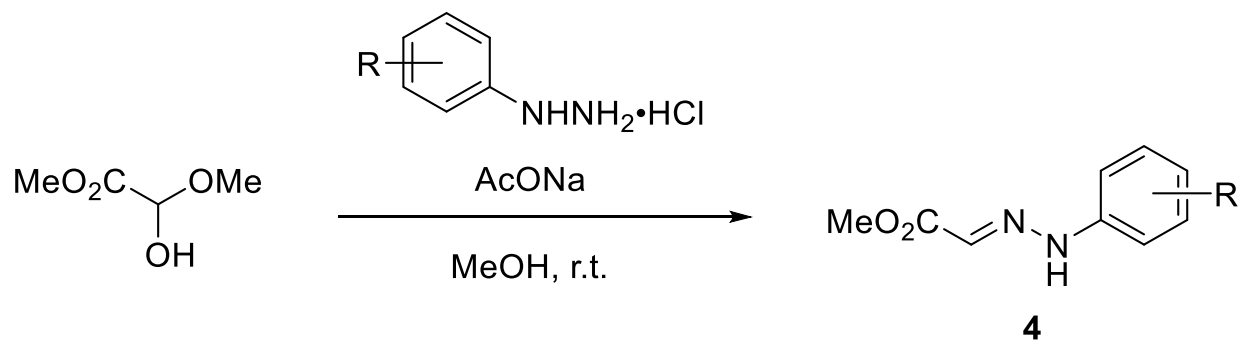

To a mixture of methyl 2-hydroxy-2-methoxyacetate $(1.0 \mathrm{~g}, 8.3 \mathrm{mmol})$ and sodium acetate $(717 \mathrm{mg}$, $8.7 \mathrm{mmol})$ in $\mathrm{MeOH}(20 \mathrm{~mL})$ was added corresponding aryl hydrazine hydrochlorides $(8.7 \mathrm{mmol})$ at room temperature. After being stirred for $0.5-2.0 \mathrm{~h}$, the reaction mixture was concentrated under reduced pressure, diluted with $\mathrm{H}_{2} \mathrm{O}(10 \mathrm{~mL})$ and extracted with $\mathrm{CHCl}_{3}(50 \mathrm{~mL} \times 3)$. The organic phase was dried over $\mathrm{MgSO}_{4}$ and concentrated under reduced pressure. The crude product was purified by recrystallization ( $n$-hexane-EtOAc) to give the corresponding hydrazones 4 .<smiles>CC(=O)C=NNc1ccc(C)cc1</smiles> 
(E)-2-[2-(4-Methylphenyl)hydrazinylidene]acetic acid methyl ester (4B) 1.18 g, 74\% yield; A yellow solid; Mp: $172-173{ }^{\circ} \mathrm{C}$ ( $n$-hexane-EtOAc); IR (KBr): 3256, $1698 \mathrm{~cm}^{-1} ;{ }^{1} \mathrm{H}$ NMR (300 MHz, DMSO- $\left.d_{6}\right) \delta: 11.22(\mathrm{br} \mathrm{s}, 1 \mathrm{H}), 7.19(\mathrm{~s}, 1 \mathrm{H}), 7.10(\mathrm{~d}, J=8.7 \mathrm{~Hz}, 2 \mathrm{H}), 7.02(\mathrm{~d}, J=8.7 \mathrm{~Hz}, 2 \mathrm{H}), 3.72$ (s, 3H), $2.24(\mathrm{~s}, 3 \mathrm{H}) ;{ }^{13} \mathrm{C}$ NMR (75 MHz, DMSO-d $\left.d_{6}\right) \delta: 164.3,141.1,130.2,129.7,123.9,113.2$, 51.2, 20.3; HRMS (ESI) m/z: [M+ Na] Calcd for $\mathrm{C}_{10} \mathrm{H}_{12} \mathrm{~N}_{2} \mathrm{O}_{2} \mathrm{Na} 215.0791$; Found 215.0792.<smiles>COC(=O)C=NNc1ccc(C(C)(C)C)cc1</smiles>

(E)-2-[2-[4-(1,1-Dimethylethyl)phenyl]hydrazinylidene]acetic acid methyl ester (4C) $1.29 \mathrm{~g}$, $67 \%$ yield; A yellow solid; Mp: $178-179{ }^{\circ} \mathrm{C}$ ( $n$-hexane-EtOAc); IR (KBr): $3272,1707 \mathrm{~cm}^{-1} ;{ }^{1} \mathrm{H}$ NMR (300 MHz, $\left.\mathrm{CDCl}_{3}\right) \delta: 8.57$ (br s, 1H), $7.30(\mathrm{~d}, J=8.7 \mathrm{~Hz}, 2 \mathrm{H}), 7.10(\mathrm{~d}, J=8.7 \mathrm{~Hz}, 2 \mathrm{H}), 7.07$ (s, 1H), 3.84 (s, 3H), 1.29 (s, 9H); ${ }^{13} \mathrm{C} \mathrm{NMR}\left(75 \mathrm{MHz}, \mathrm{CDCl}_{3}\right) \delta: 164.8,145.5,139.9,126.1,124.6$, 113.7, 51.9, 34.2, 31.4; HRMS (ESI) m/z: $[\mathrm{M}+\mathrm{Na}]^{+}$Calcd for $\mathrm{C}_{13} \mathrm{H}_{18} \mathrm{~N}_{2} \mathrm{O}_{2} \mathrm{Na}$ 257.1261; Found 257.1258 .<smiles>COC(=O)C=NNc1ccccc1</smiles>

(E)-2-(2-Phenylhydrazinylidene)acetic acid methyl ester (4D) $746 \mathrm{mg}, 50 \%$ yield; A white solid; Mp: $129-130{ }^{\circ} \mathrm{C}$ ( $n$-hexane-EtOAc); IR (KBr): 3273, $1699 \mathrm{~cm}^{-1}$; ${ }^{1} \mathrm{H}$ NMR (300 MHz, DMSO- $\left.d_{6}\right) \delta$ : 11.30 (br s, 1H), 7.30 (d, $J=7.5 \mathrm{~Hz}, 2 \mathrm{H}), 7.23(\mathrm{~s}, 1 \mathrm{H}), 7.14(\mathrm{~s}, 1 \mathrm{H}), 7.11(\mathrm{~m}, 1 \mathrm{H}), 6.93(\mathrm{t}, J=7.5 \mathrm{~Hz}$, $1 \mathrm{H}), 3.73$ (s, 3H); ${ }^{13} \mathrm{C}$ NMR (75 MHz, $\mathrm{CDCl}_{3}$ ) $\delta: 164.9,142.4,129.3,125.1,122.4,114.0,51.9$; HRMS (ESI) m/z: [M + Na] $]^{+}$Calcd for $\mathrm{C}_{9} \mathrm{H}_{10} \mathrm{~N}_{2} \mathrm{O}_{2} \mathrm{Na}$ 201.0635; Found 201.0636.<smiles>COC(=O)C=NNc1ccc(F)cc1</smiles>

(E)-2-[2-(4-Fluorophenyl)hydrazinylidene]acetic acid methyl ester (4E) $932 \mathrm{mg}, 57 \%$ yield; A yellow solid; Mp: $140-150{ }^{\circ} \mathrm{C}$ ( $n$-hexane-EtOAc); IR (KBr): 3267, $1708 \mathrm{~cm}^{-1} ;{ }^{1} \mathrm{H}$ NMR (300 MHz, $\left.\mathrm{CDCl}_{3}\right) \delta: 8.66($ br s, $1 \mathrm{H}), 7.11-7.07(\mathrm{~m}, 3 \mathrm{H}), 6.96(\mathrm{t}, J=8.7 \mathrm{~Hz}, 2 \mathrm{H}), 3.83(\mathrm{~s}, 3 \mathrm{H}) ;{ }^{13} \mathrm{C}$ NMR $(150$ $\left.\mathrm{MHz}, \mathrm{CDCl}_{3}\right) \delta: 164.8,159.4\left(\mathrm{C}-\mathrm{F},{ }^{1} J_{\mathrm{C}-\mathrm{F}}=239.3 \mathrm{~Hz}\right), 157.8\left(\mathrm{C}-\mathrm{F},{ }^{1} J_{\mathrm{C}-\mathrm{F}}=239.3 \mathrm{~Hz}\right), 138.7(\mathrm{C}-\mathrm{F}$, $\left.{ }^{4} J_{\mathrm{C}-\mathrm{F}}=2.3 \mathrm{~Hz}\right), 138.7\left(\mathrm{C}-\mathrm{F},{ }^{4} J_{\mathrm{C}-\mathrm{F}}=2.3 \mathrm{~Hz}\right), 125.2,116.1\left(\mathrm{C}-\mathrm{F},{ }^{2} J_{\mathrm{C}-\mathrm{F}}=22.8 \mathrm{~Hz}\right), 115.9\left(\mathrm{C}-\mathrm{F},{ }^{2} J_{\mathrm{C}-\mathrm{F}}=\right.$ $22.8 \mathrm{~Hz}), 115.2\left(\mathrm{C}-\mathrm{F},{ }^{3} J_{\mathrm{C}-\mathrm{F}}=7.8 \mathrm{~Hz}\right), 115.2\left(\mathrm{C}-\mathrm{F},{ }^{3} J_{\mathrm{C}-\mathrm{F}}=7.8 \mathrm{~Hz}\right), 52.0 ; \mathrm{HRMS}(\mathrm{ESI}) \mathrm{m} / \mathrm{z}:[\mathrm{M}+\mathrm{Na}]^{+}$ Calcd for $\mathrm{C}_{9} \mathrm{H}_{9} \mathrm{~N}_{2} \mathrm{O}_{2} \mathrm{FNa} 219.0540$; Found 219.0538.<smiles>CC(=O)C=NNc1ccc(Cl)cc1</smiles> 
(E)-2-[2-(4-Chlorophenyl)hydrazinylidene]acetic acid methyl ester (4F) $644 \mathrm{mg}, 36 \%$ yield; A yellow solid; Mp: $173-177^{\circ} \mathrm{C}$ ( $n$-hexane-EtOAc); IR (KBr): 3260, $1701 \mathrm{~cm}^{-1} ;{ }^{1} \mathrm{H}$ NMR (300 MHz, DMSO- $\left.d_{6}\right) \delta: 11.37$ (br s, 1H), 7.34 (d, $\left.J=8.7 \mathrm{~Hz}, 2 \mathrm{H}\right), 7.23$ (s, 1H), $7.11(\mathrm{~d}, J=8.7 \mathrm{~Hz}, 2 \mathrm{H}), 3.74$ (s, 3H); ${ }^{13} \mathrm{C}$ NMR (75 MHz, DMSO- $\left.d_{6}\right) \delta: 164.0,142.4,129.2,125.6,124.8,114.7,51.3$; HRMS (ESI) $\mathrm{m} / \mathrm{z}:[\mathrm{M}+\mathrm{Na}]^{+}$Calcd for $\mathrm{C}_{9} \mathrm{H}_{9} \mathrm{~N}_{2} \mathrm{O}_{2}{ }^{35} \mathrm{ClNa}$ 235.0245; Found 235.0246.<smiles>CC(=O)C=NNc1ccc(Br)cc1</smiles>

(E)-2-[2-(4-Bromophenyl)hydrazinylidene]acetic acid methyl ester (4G) 1.27 g, 59\% yield; A yellow solid; Mp: $186-187^{\circ} \mathrm{C}$ ( $n$-hexane-EtOAc); IR (KBr): 3258, $1704 \mathrm{~cm}^{-1} ;{ }^{1} \mathrm{H}$ NMR (300 MHz, DMSO- $\left.d_{6}\right) \delta: 11.4(\mathrm{br} \mathrm{s}, 1 \mathrm{H}), 7.46(\mathrm{~d}, J=8.7 \mathrm{~Hz}, 2 \mathrm{H}), 7.22(\mathrm{~s}, 1 \mathrm{H}), 7.06(\mathrm{~d}, J=8.7 \mathrm{~Hz}, 2 \mathrm{H}), 3.73(\mathrm{~s}$, $3 \mathrm{H}) ;{ }^{13} \mathrm{C}$ NMR (75 MHz, DMSO- $\left.d_{6}\right) \delta: 164.0,142.7,132.0,125.7,115.2,112.5,51.4$; HRMS (ESI) $\mathrm{m} / \mathrm{z}:[\mathrm{M}+\mathrm{Na}]^{+} \mathrm{Calcd}$ for $\mathrm{C}_{9} \mathrm{H}_{9} \mathrm{~N}_{2} \mathrm{O}_{2}{ }^{79} \mathrm{BrNa} 278.9740$; Found 278.9740 .<smiles>COc1ccccc1N/N=C/C(OC)OC</smiles>

(E)-2-[2-(2-Methoxyphenyl)hydrazinylidene]acetic acid methyl ester (4H) 1.02 g, 59\% yield; A yellow solid; Mp: 119-120 ${ }^{\circ} \mathrm{C}$ ( $n$-hexane-EtOAc); IR (KBr): 3237, $1717 \mathrm{~cm}^{-1} ;{ }^{1} \mathrm{H}$ NMR (300 MHz, DMSO- $\left.d_{6}\right) \delta: 11.38(\mathrm{br} \mathrm{s}, 1 \mathrm{H}), 7.46(\mathrm{~d}, J=9.0 \mathrm{~Hz}, 2 \mathrm{H}), 7.23(\mathrm{~s}, 1 \mathrm{H}), 7.06(\mathrm{~d}, J=9.0 \mathrm{~Hz}, 2 \mathrm{H}), 3.73$ (s, 3H), 3.84 (s, 3H); ${ }^{13} \mathrm{C}$ NMR (75 MHz, DMSO-d $)$ ) $: 164.7,145.7,131.6,125.9,121.8,121.5$, 113.8, 110.2, 55.5, 51.8; HRMS (ESI) m/z: $[\mathrm{M}+\mathrm{Na}]^{+}$Calcd for $\mathrm{C}_{10} \mathrm{H}_{12} \mathrm{~N}_{2} \mathrm{O}_{3} \mathrm{Na}$ 231.0740; Found 231.0736 .

\subsection{Preperatioin of hydrazones (General procedure-II).}

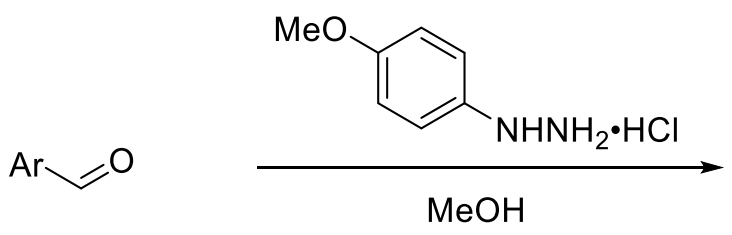<smiles>COc1ccc(N/N=C/[Ga])cc1</smiles>

To a solution of corresponding aryl aldehydes $(1.0 \mathrm{~g})$ in $\mathrm{MeOH}(20 \mathrm{~mL})$ was added 4-methoxyphenylhydrazine hydrochloride (1.0 equiv.) at room temperature. After being stirred for $3.0 \mathrm{~h}$, the reaction mixture was filtered and rinsed with cold $\mathrm{MeOH}(5 \mathrm{~mL})$. The crude product was purified by recrystallization $(\mathrm{MeOH})$ to give the corresponding hydrazones 4 . 
<smiles>COc1ccc(N/N=C/c2ccc(C)cc2)cc1</smiles>

(E)-4-Methylbenzaldehyde 2-(4-methoxyphenyl)hydrazone (4K) 1.20 g, 60\% yield; A yellow solid; Mp: 129-133 ${ }^{\circ} \mathrm{C}(\mathrm{MeOH})$; IR (KBr): $3321 \mathrm{~cm}^{-1}$; ${ }^{1} \mathrm{H}$ NMR (300 MHz, DMSO-d $\left.d_{6}\right) \delta: 10.02$ (br s, 1H), $7.80(\mathrm{~s}, 1 \mathrm{H}), 7.52$ (d, $J=8.1 \mathrm{~Hz}, 2 \mathrm{H}), 7.18(\mathrm{~d}, J=8.1 \mathrm{~Hz}, 2 \mathrm{H}), 7.02(\mathrm{~d}, J=9.0 \mathrm{~Hz}, 2 \mathrm{H}), 6.84$ (d, $J=9.0 \mathrm{~Hz}, 2 \mathrm{H}), 3.69$ (s, 3H), 2.30 (s, 3H); ${ }^{13} \mathrm{C}$ NMR (75 MHz, DMSO-d 6 ) $\delta: 152.5,139.5,137.0$, 135.5, 133.3, 129.2, 125.4, 114.6, 112.9, 55.2, 20.9; HRMS (ESI) m/z: $[\mathrm{M}+\mathrm{H}]^{+}$Calcd for $\mathrm{C}_{15} \mathrm{H}_{17} \mathrm{~N}_{2} \mathrm{O} 241.1335$; Found 241.1335.<smiles>COc1ccc(N/N=C/c2ccc(C(F)(F)F)cc2)cc1</smiles>

(E)-4-(Trifluoromethyl)benzaldehyde 2-(4-methoxyphenyl)hydrazone (4N) 1.00 g, 59\% yield; Yellow crystals; Mp: $149-151{ }^{\circ} \mathrm{C}(\mathrm{MeOH})$; IR (KBr): $3296 \mathrm{~cm}^{-1}$; ${ }^{1} \mathrm{H}$ NMR (300 MHz, DMSO- $\left.d_{6}\right) \delta$ : 10.49 (br s, 1H), 7.89 (s, 1H), 7.83 (d, $J=8.1 \mathrm{~Hz}, 2 \mathrm{H}), 7.70$ (d, $J=8.4 \mathrm{~Hz}, 2 \mathrm{H}), 7.11$ (d, $J=9.3 \mathrm{~Hz}$, 2H), $6.90(\mathrm{~d}, J=9.0 \mathrm{~Hz}, 2 \mathrm{H}), 3.72(\mathrm{~s}, 3 \mathrm{H}) ;{ }^{13} \mathrm{C}$ NMR (150 MHz, DMSO- $\left.d_{6}\right) \delta: 153.0,140.0,138.7$, 133.1, $127.4\left(\mathrm{C}-\mathrm{F},{ }^{2} J_{\mathrm{C}-\mathrm{F}}, J=31.4 \mathrm{~Hz}\right), 127.2\left(\mathrm{C}-\mathrm{F},{ }^{2} J_{\mathrm{C}-\mathrm{F}}, J=31.4 \mathrm{~Hz}\right), 127.1\left(\mathrm{C}-\mathrm{F},{ }^{1} J_{\mathrm{C}-\mathrm{F}}, J=269.9\right.$ $\mathrm{Hz}), 127.0\left(\mathrm{C}-\mathrm{F},{ }^{2} J_{\mathrm{C}-\mathrm{F}}, J=31.4 \mathrm{~Hz}\right), 126.8\left(\mathrm{C}-\mathrm{F},{ }^{2} J_{\mathrm{C}-\mathrm{F}}, J=31.4 \mathrm{~Hz}\right), 125.6,125.4\left(\mathrm{C}-\mathrm{F},{ }^{3} J_{\mathrm{C}-\mathrm{F}}, J=3.7\right.$ $\mathrm{Hz}$ ), $125.4\left(\mathrm{C}-\mathrm{F},{ }^{3} J_{\mathrm{C}-\mathrm{F}}, J=3.7 \mathrm{~Hz}\right), 125.4\left(\mathrm{C}-\mathrm{F},{ }^{3} J_{\mathrm{C}-\mathrm{F}}, J=3.7 \mathrm{~Hz}\right), 125.3\left(\mathrm{C}-\mathrm{F},{ }^{1} J_{\mathrm{C}-\mathrm{F}}=269.9 \mathrm{~Hz}\right)$, $123.5\left(\mathrm{C}-\mathrm{F},{ }^{1} J_{\mathrm{C}-\mathrm{F}}=269.9 \mathrm{~Hz}\right), 121.7\left(\mathrm{C}-\mathrm{F},{ }^{1} J_{\mathrm{C}-\mathrm{F}}=269.9 \mathrm{~Hz}\right), 114.7,113.3,55.2 ; \mathrm{HRMS}(\mathrm{ESI}) \mathrm{m} / \mathrm{z}$ : $[\mathrm{M}+\mathrm{H}]^{+}$Calcd for $\mathrm{C}_{15} \mathrm{H}_{14} \mathrm{~N}_{2} \mathrm{OF}_{3} 295.1053$; Found 295.1053.<smiles>COc1ccc(N/N=C/c2cccc(Cl)c2)cc1</smiles>

(E)-3-Chlorobenzaldehyde 2-(4-methoxyphenyl)hydrazone (4P) $904 \mathrm{mg}, 49 \%$ yield; A yellow solid; Mp: 122-124 ${ }^{\circ} \mathrm{C}(\mathrm{MeOH})$; IR (KBr): $3297 \mathrm{~cm}^{-1} ;{ }^{1} \mathrm{H}$ NMR (300 MHz, DMSO- $d_{6}$ ) $\delta: 10.33$ (br s, 1H), 7.79 (s, 1H), 7.68 (s, 1H), 7.57 (d, $J=7.5 \mathrm{~Hz}, 1 \mathrm{H}), 7.39$ (t, $J=7.8 \mathrm{~Hz}, 1 \mathrm{H}), 7.03$ (d, $J=7.8 \mathrm{~Hz}$, 1H), $7.06(\mathrm{~d}, J=9.0 \mathrm{~Hz}, 2 \mathrm{H}), 6.86(\mathrm{~d}, J=9.0 \mathrm{~Hz}, 2 \mathrm{H}), 3.70(\mathrm{~s}, 3 \mathrm{H}) ;{ }^{13} \mathrm{C}$ NMR (75 MHZ, DMSO- $\left.d_{6}\right)$ $\delta: 152.9,139.0,138.4,133.5,133.3,130.4,127.0,124.5,123.9,114.6,113.2,55.2$; HRMS (ESI) $\mathrm{m} / \mathrm{z}:[\mathrm{M}+\mathrm{H}]^{+}$Calcd for $\mathrm{C}_{14} \mathrm{H}_{14} \mathrm{~N}_{2} \mathrm{O}^{35} \mathrm{Cl}$ 261.0789; Found 261.0790.<smiles>COc1ccc(N/N=C/c2ccccc2O)cc1</smiles>

(E)-2-Hydroxybenzaldehyde 2-(4-methoxyphenyl)hydrazone (4Q) 1.05 g, 53\% yield; Yellow crystals; Mp: $130-132{ }^{\circ} \mathrm{C}(\mathrm{MeOH})$; IR (KBr): $3331 \mathrm{~cm}^{-1}$; ${ }^{1} \mathrm{H}$ NMR $\left(300 \mathrm{MHz}, \mathrm{CDCl}_{3}\right) \delta: 10.9$ (br s, 1H), $7.75(\mathrm{~s}, 1 \mathrm{H}), 7.34$ (br s, 1H), 7.24-7.18 (m, 1H), 7.09 (d, $J=7.5 \mathrm{~Hz}, 1 \mathrm{H}), 6.98(\mathrm{~d}, J=8.4 \mathrm{~Hz}$, 
1H), 6.93-6.83 (m, 5H), $3.76(\mathrm{~s}, 3 \mathrm{H}) ;{ }^{13} \mathrm{C} \mathrm{NMR}\left(75 \mathrm{MHz}, \mathrm{CDCl}_{3}\right) \delta: 156.9,154.3,140.6,137.4$, 129.7, 129.1, 119.4, 118.6, 116.5, 114.9, 114.2, 55.6; HRMS (ESI) $\mathrm{m} / \mathrm{z}:[\mathrm{M}+\mathrm{H}]^{+}$Calcd for $\mathrm{C}_{14} \mathrm{H}_{15} \mathrm{~N}_{2} \mathrm{O}_{2} 243.1128$; Found 243.1129.<smiles>COc1ccc(N/N=C/c2ccc3ccccc3c2)cc1</smiles>

(E)-2-Naphthaldehyde 2-(4-methoxyphenyl)hydrazone (4R) $1.44 \mathrm{~g}, 82 \%$ yield; A yellow solid; Mp: $173-175^{\circ} \mathrm{C}(\mathrm{MeOH}) ; \mathrm{IR}(\mathrm{KBr}): 3304 \mathrm{~cm}^{-1} ;{ }^{1} \mathrm{H}$ NMR (300 MHz, DMSO- $\left.d_{6}\right) \delta: 10.26$ (br s, $1 \mathrm{H}$ ), 7.99-7.87 (m, 6H), 7.54-7.45 (m, 2H), 7.07 (d, $J=8.7 \mathrm{~Hz}, 2 \mathrm{H}), 6.87$ (d, $J=9.0 \mathrm{~Hz}, 2 \mathrm{H}), 3.71(\mathrm{~s}$, $3 \mathrm{H}) ;{ }^{13} \mathrm{C}$ NMR (75 MHz, DMSO-d $)$ ) $\delta$ : 152.7, 139.3, 135.3, 133.8, 133.2, 132.6, 128.1, 127.7, 127.6, 126.4, 125.8, 125.3, 122.4, 114.7, 113.0, 55.3; HRMS (ESI) m/z: $[\mathrm{M}+\mathrm{H}]^{+}$Calcd for $\mathrm{C}_{18} \mathrm{H}_{17} \mathrm{~N}_{2} \mathrm{O}$ 277.1335; Found 277.1330.

\subsection{General procedure for synthesis of pyrazoles 2 (self-condensation).}<smiles>[R]c1ccc(N/N=C/C=C/C(=O)OCC)cc1</smiles>

1

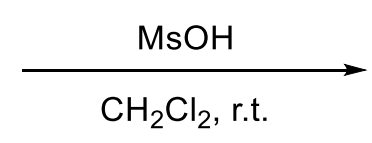

$\mathrm{CH}_{2} \mathrm{Cl}_{2}$, r.t.<smiles>[R]c1ccc(-n2cc(CCOC(=O)OCC)c(/C=C\C(=O)OCC)n2)cc1</smiles>

2

To a solution of conjugated hydrazones $1(0.20 \mathrm{mmol})$ in $\mathrm{CH}_{2} \mathrm{Cl}_{2}(1.0 \mathrm{~mL})$ was added $\mathrm{MsOH}(13 \mu \mathrm{L}$, $0.20 \mathrm{mmol}$ ) at room temperature. After being stirred at the same temperature for several hours, the reaction mixture was quenched with saturated aqueous $\mathrm{NaHCO}_{3}(5 \mathrm{~mL})$ and extracted with $\mathrm{CHCl}_{3}$ $(30 \mathrm{~mL} \times 3)$. The organic phase was dried over $\mathrm{MgSO}_{4}$ and concentrated under reduced pressure. The crude product was purified by preparative TLC ( $n$-hexane: EtOAc) to afford the corresponding pyrazoles $\mathbf{2}$. Conjugated hydrazones $\mathbf{1} \mathbf{a}-\mathbf{j}$ were prepared according to a published procedure. ${ }^{1 a}$ The spectral data were in agreement with literature values. ${ }^{\text {la }}$<smiles>CCOC(=O)C=Cc1nn(-c2ccc(OC)cc2)cc1CCC(=O)OCC</smiles>

(E)-3-[4-(2-Ethoxy-2-oxoethyl)-1-(4-methoxyphenyl)-1 $H$-pyrazol-3-yl]-2-propenoic acid ethyl ester (2a) $30.2 \mathrm{mg}, 84 \%$ yield; Reaction time: $1 \mathrm{~h}$; Purification by preparative TLC ( $n$-hexane: EtOAc $=2: 1)$; A yellow oil; IR (neat): $1738,1710 \mathrm{~cm}^{-1} ;{ }^{1} \mathrm{H}$ NMR $\left(500 \mathrm{MHz}, \mathrm{CDCl}_{3}\right) \delta: 7.89(\mathrm{~s}, 1 \mathrm{H})$, $7.68(\mathrm{~d}, J=16.0 \mathrm{~Hz}, 1 \mathrm{H}), 7.60(\mathrm{~d}, J=9.0 \mathrm{~Hz}, 2 \mathrm{H}), 6.96(\mathrm{~d}, J=9.0 \mathrm{~Hz}, 2 \mathrm{H}), 6.67$ (d, $J=16.0 \mathrm{~Hz}$, $1 \mathrm{H}), 4.26$ (q, $J=7.0 \mathrm{~Hz}, 2 \mathrm{H}), 4.20$ (q, $J=7.0 \mathrm{~Hz}, 2 \mathrm{H}), 3.84$ (s, 3H), 3.65 (s, 2H), 1.33 (t, $J=7.0 \mathrm{~Hz}$, $3 \mathrm{H}), 1.29(\mathrm{t}, J=7.0 \mathrm{~Hz}, 3 \mathrm{H}) ;{ }^{13} \mathrm{C} \mathrm{NMR}\left(125 \mathrm{MHz}, \mathrm{CDCl}_{3}\right) \delta: 170.7,167.1,158.6,146.4,134.1$, 
133.4, 127.9, 120.7, 119.4, 115.7, 114.5, 61.2, 60.4, 55.6, 29.9, 14.3, 14.1; HRMS (ESI) m/z: [M + $\mathrm{Na}]^{+}$Calcd for $\mathrm{C}_{19} \mathrm{H}_{22} \mathrm{~N}_{2} \mathrm{O}_{5} \mathrm{Na} 381.1421$; Found 381.1421.<smiles>CCOC/C=C/c1nn(-c2ccc(C)cc2)cc1CCOCC</smiles>

(E)-3-[4-(2-Ethoxy-2-oxoethyl)-1-(4-methylphenyl)-1 H-pyrazol-3-yl]-2-propenoic acid ethyl ester (2b) $21.4 \mathrm{mg}, 63 \%$ yield; Reaction time: $1 \mathrm{~h}$; Purification by preparative TLC ( $n$-hexane: EtOAc = 2:1); A yellow solid; Mp: $76-78{ }^{\circ} \mathrm{C}$; IR (neat): $1738,1710 \mathrm{~cm}^{-1} ;{ }^{1} \mathrm{H}$ NMR $(300 \mathrm{MHz}$, $\left.\mathrm{CDCl}_{3}\right) \delta: 7.92(\mathrm{~s}, 1 \mathrm{H}), 7.66(\mathrm{~d}, J=15.9 \mathrm{~Hz}, 1 \mathrm{H}), 7.56(\mathrm{~d}, J=8.7 \mathrm{~Hz}, 2 \mathrm{H}), 7.22(\mathrm{~d}, J=8.7 \mathrm{~Hz}, 2 \mathrm{H})$, $6.66(\mathrm{~d}, J=15.9 \mathrm{~Hz}, 1 \mathrm{H}), 4.25(\mathrm{q}, J=7.2 \mathrm{~Hz}, 2 \mathrm{H}), 4.19$ (q, $J=7.2 \mathrm{~Hz}, 2 \mathrm{H}), 3.65$ (s, 2H), 2.38 (s, $3 \mathrm{H}), 1.33(\mathrm{t}, J=7.2 \mathrm{~Hz}, 3 \mathrm{H}), 1.29(\mathrm{t}, J=7.2 \mathrm{~Hz}, 3 \mathrm{H}) ;{ }^{13} \mathrm{C} \mathrm{NMR}\left(75 \mathrm{MHz}, \mathrm{CDCl}_{3}\right) \delta: 170.5,166.9$, $146.4,137.3,136.7,133.9,129.8,127.7,119.5,119.0,115.7,61.2,60.4,30.0,21.0,14.4,14.2$; HRMS (ESI) m/z: [M + Na] $]^{+}$Calcd for $\mathrm{C}_{19} \mathrm{H}_{22} \mathrm{~N}_{2} \mathrm{O}_{4} \mathrm{Na}$ 365.1472; Found 365.1470.<smiles>CCOC(=O)C=Cc1nn(-c2ccc(C(C)(C)C)cc2)cc1CCC(=O)OCC</smiles>

(E)-3-[1-[4-(1,1-Dimethyl)phenyl]-4-(2-ethoxy-2-oxoethyl)-1 $H$-pyrazol-3-yl]-2-propenoic acid ethyl ester (2c) $32.1 \mathrm{mg}, 83 \%$ yield; Reaction time: $1 \mathrm{~h}$; Purification by preparative TLC ( $n$-hexane: EtOAc = 2:1); A yellow solid; Mp: $70{ }^{\circ} \mathrm{C}$ (decomposed); IR (neat): 1738, $1712 \mathrm{~cm}^{-1} ;{ }^{1} \mathrm{H}$ NMR $(300$ $\left.\mathrm{MHz}, \mathrm{CDCl}_{3}\right) \delta: 7.93(\mathrm{~s}, 1 \mathrm{H}), 7.66(\mathrm{~d}, J=15.9 \mathrm{~Hz}, 1 \mathrm{H}), 7.59(\mathrm{~d}, J=8.7 \mathrm{~Hz}, 2 \mathrm{H}), 7.44$ (d, $J=8.7 \mathrm{~Hz}$, 2H), 6.66 (d, $J=15.9 \mathrm{~Hz}, 1 \mathrm{H}), 4.26$ (q, $J=7.2 \mathrm{~Hz}, 2 \mathrm{H}), 4.19$ (q, $J=7.2 \mathrm{~Hz}, 2 \mathrm{H}), 3.65$ (s, 2H), 1.34 (s, 9H), 1.33 (t, $J=7.2 \mathrm{~Hz}, 3 \mathrm{H}), 1.29$ (t, $J=7.2 \mathrm{~Hz}, 3 \mathrm{H}) ;{ }^{13} \mathrm{C} \mathrm{NMR}\left(75 \mathrm{MHz}, \mathrm{CDCl}_{3}\right) \delta: 170.5,166.9$, 150.0, 146.4, 137.1, 134.0, 127.7, 126.2, 119.5, 118.8, 115.7, 61.2, 60.4, 34.6, 31.4, 30.0, 14.4, 14.2; HRMS (ESI) m/z: [M + Na] $]^{+}$Calcd for $\mathrm{C}_{22} \mathrm{H}_{28} \mathrm{~N}_{2} \mathrm{O}_{4} \mathrm{Na}$ 407.1941; Found 407.1942.<smiles>CCOCC=Cc1nn(-c2ccc(C#N)cc2)cc1CCOCC</smiles>

(E)-3-[1-(4-Cyanophenyl)-4-(2-ethoxy-2-oxoethyl)-1 $H$-pyrazol-3-yl]-2-propenoic acid ethyl ester (2d) $9.5 \mathrm{mg}, 27 \%$ yield; Reaction time: $18 \mathrm{~h}$; Purification by preparative TLC ( $n$-hexane: EtOAc = 2:1); White crystals; Mp: 139-140 ${ }^{\circ} \mathrm{C}$ ( $n$-hexane-EtOAc); IR (KBr): 2220, 1740, $1710 \mathrm{~cm}^{-1}$; ${ }^{1} \mathrm{H}$ NMR (300 MHz, $\left.\mathrm{CDCl}_{3}\right) \delta: 8.06(\mathrm{~s}, 1 \mathrm{H}), 7.84(\mathrm{~d}, J=9.0 \mathrm{~Hz}, 2 \mathrm{H}), 7.73(\mathrm{~d}, J=9.0 \mathrm{~Hz}, 2 \mathrm{H}), 7.61$ $(\mathrm{d}, J=15.9 \mathrm{~Hz}, 1 \mathrm{H}), 6.70(\mathrm{~d}, J=16.2 \mathrm{~Hz}, 1 \mathrm{H}), 4.27$ (q, $J=7.2 \mathrm{~Hz}, 2 \mathrm{H}), 4.20$ (q, $J=7.2 \mathrm{~Hz}, 2 \mathrm{H})$, $3.66(\mathrm{~s}, 2 \mathrm{H}), 1.34$ (t, $J=7.2 \mathrm{~Hz}, 3 \mathrm{H}), 1.30(\mathrm{t}, J=7.2 \mathrm{~Hz}, 3 \mathrm{H}) ;{ }^{13} \mathrm{C} \mathrm{NMR}\left(75 \mathrm{MHz}, \mathrm{CDCl}_{3}\right) \delta: 170.2$, 
166.5, 148.1, 142.2, 133.6, 133.0, 127.7, 121.1, 118.8, 118.1, 117.1, 110.1, 61.4, 60.7, 29.8, 14.4, 14.3; HRMS (ESI) m/z: [M + H] $]^{+}$Calcd for $\mathrm{C}_{19} \mathrm{H}_{20} \mathrm{~N}_{3} \mathrm{O}_{4} 376.1268$; Found 376.1268 .<smiles>CCOCCc1nn(-c2ccccc2)cc1C=CCC(=O)OCC</smiles>

(E)-3-[4-(2-Ethoxy-2-oxoethyl)-1-phenyl-1 H-pyrazol-3-yl]-2-propenoic acid ethyl ester (2e) 9.7 mg, 30\% yield; Reaction time: $2 \mathrm{~h}$; Purification by preparative TLC ( $n$-hexane: EtOAc $=2: 1)$; A yellow solid; Mp: $76{ }^{\circ} \mathrm{C}$ (decomposed); IR (neat): 1731, $1709 \mathrm{~cm}^{-1}$; ${ }^{1} \mathrm{H}$ NMR $\left(300 \mathrm{MHz}, \mathrm{CDCl}_{3}\right) \delta$ : $7.97(\mathrm{~s}, 1 \mathrm{H}), 7.70-7.67(\mathrm{~m}, 2 \mathrm{H}), 7.66(\mathrm{~d}, J=15.9 \mathrm{~Hz}, 1 \mathrm{H}), 7.46-7.41(\mathrm{~m}, 2 \mathrm{H}), 7.32(\mathrm{~m}, 1 \mathrm{H}), 6.67(\mathrm{~d}$, $J=15.9 \mathrm{~Hz}, 1 \mathrm{H}), 4.26(\mathrm{q}, J=7.2 \mathrm{~Hz}, 2 \mathrm{H}), 4.19$ (q, $J=7.2 \mathrm{~Hz}, 2 \mathrm{H}), 3.66(\mathrm{~s}, 2 \mathrm{H}), 1.33$ (t, $J=7.2 \mathrm{~Hz}$, $3 \mathrm{H}), 1.29(\mathrm{t}, J=7.2 \mathrm{~Hz}, 3 \mathrm{H}) ;{ }^{13} \mathrm{C} \mathrm{NMR}\left(75 \mathrm{MHz}, \mathrm{CDCl}_{3}\right) \delta: 170.7,167.0,146.8,139.6,134.0,129.4$, $127.8,127.0,119.8,119.1,116.0,61.3,60.5,29.9,14.3,14.1$; HRMS (ESI) m/z: $[\mathrm{M}+\mathrm{Na}]^{+} \mathrm{Calcd}$ for $\mathrm{C}_{18} \mathrm{H}_{20} \mathrm{~N}_{2} \mathrm{O}_{4} \mathrm{Na} 351.1315$; Found 351.1312 .<smiles>CCOC(=O)C=Cc1nn(-c2ccc(F)cc2)cc1CC=O</smiles>

(E)-3-[4-(2-Ethoxy-2-oxoethyl)-1-(4-fluorophenyl)-1H-pyrazol-3-yl]-2-propenoic acid ethyl ester (2f) $27.9 \mathrm{mg}, 81 \%$ yield; Reaction time: $2 \mathrm{~h}$; Purification by preparative TLC ( $n$-hexane: EtOAc = 2:1); A yellow solid; Mp: $70{ }^{\circ} \mathrm{C}$ (decomposed); IR (neat): 1736, $1711 \mathrm{~cm}^{-1} ;{ }^{1} \mathrm{H}$ NMR $(300$ $\left.\mathrm{MHz}, \mathrm{CDCl}_{3}\right) \delta: 7.91(\mathrm{~s}, 1 \mathrm{H}), 7.67-7.62(\mathrm{~m}, 2 \mathrm{H}), 7.64(\mathrm{~d}, J=15.9 \mathrm{~Hz}, 1 \mathrm{H}), 7.17-7.09(\mathrm{~m}, 2 \mathrm{H}), 6.66$ $(\mathrm{d}, J=15.9 \mathrm{~Hz}, 1 \mathrm{H}), 4.26$ (q, $J=7.2 \mathrm{~Hz}, 2 \mathrm{H}), 4.19$ (q, $J=7.2 \mathrm{~Hz}, 2 \mathrm{H}), 3.64(\mathrm{~s}, 2 \mathrm{H}), 1.33$ (t, $J=7.2$ $\mathrm{Hz}, 3 \mathrm{H}), 1.29(\mathrm{t}, J=7.2 \mathrm{~Hz}, 3 \mathrm{H}) ;{ }^{13} \mathrm{C} \mathrm{NMR}\left(150 \mathrm{MHz}, \mathrm{CDCl}_{3}\right) \delta: 170.6,166.9,162.2\left(\mathrm{C}-\mathrm{F},{ }^{1} J_{\mathrm{C}-\mathrm{F}}=\right.$ $245.3 \mathrm{~Hz}), 160.6\left(\mathrm{C}-\mathrm{F},{ }^{1} J_{\mathrm{C}-\mathrm{F}}=245.3 \mathrm{~Hz}\right), 147.0,136.0\left(\mathrm{C}-\mathrm{F},{ }^{4} J_{\mathrm{C}-\mathrm{F}}=2.9 \mathrm{~Hz}\right), 136.0\left(\mathrm{C}-\mathrm{F},{ }^{4} J_{\mathrm{C}-\mathrm{F}}=2.9\right.$ $\mathrm{Hz}), 133.8,127.9,120.9\left(\mathrm{C}-\mathrm{F},{ }^{3} J_{\mathrm{C}-\mathrm{F}}=8.4 \mathrm{~Hz}\right), 120.9\left(\mathrm{C}-\mathrm{F},{ }^{3} J_{\mathrm{C}-\mathrm{F}}=8.4 \mathrm{~Hz}\right), 120.0,116.4\left(\mathrm{C}-\mathrm{F},{ }^{2} J_{\mathrm{C}-\mathrm{F}}=\right.$ $23.0 \mathrm{~Hz}), 116.2\left(\mathrm{C}-\mathrm{F},{ }^{2} J_{\mathrm{C}-\mathrm{F}}=23.0 \mathrm{~Hz}\right), 116.1,61.3,60.5,29.8,14.3,14.1$; HRMS (ESI) $\mathrm{m} / \mathrm{z}:[\mathrm{M}+$ $\mathrm{H}]^{+}$Calcd for $\mathrm{C}_{18} \mathrm{H}_{20} \mathrm{~N}_{2} \mathrm{O}_{4} \mathrm{~F}$ 347.1402; Found 347.1401.<smiles>CCOC/C=C/c1nn(-c2ccc(Cl)cc2)cc1CCOCCOCC</smiles>

(E)-3-[1-(4-Chlorophenyl)-4-(2-ethoxy-2-oxoethyl)-1H-pyrazol-3-yl]-2-propenoic acid ethyl ester (2g) $24.7 \mathrm{mg}$, 68\% yield; Reaction time: $2 \mathrm{~h}$; Purification by preparative TLC ( $n$-hexane: EtOAc $=2: 1)$; Pale yellow crystals; Mp: $86-88{ }^{\circ} \mathrm{C}(n$-hexane-Et $2 \mathrm{O}) ; \mathrm{IR}$ (neat): $1736,1711 \mathrm{~cm}^{-1} ;{ }^{1} \mathrm{H}$ $\operatorname{NMR}\left(300 \mathrm{MHz}, \mathrm{CDCl}_{3}\right) \delta: 7.94$ (s, 1H), $7.63(\mathrm{~d}, J=9.0 \mathrm{~Hz}, 2 \mathrm{H}), 7.62$ (d, $\left.J=15.9 \mathrm{~Hz}, 1 \mathrm{H}\right), 7.40$ (d, 
$J=9.0 \mathrm{~Hz}, 2 \mathrm{H}), 6.66(\mathrm{~d}, J=15.9 \mathrm{~Hz}, 1 \mathrm{H}), 4.26(\mathrm{q}, J=7.2 \mathrm{~Hz}, 2 \mathrm{H}), 4.19(\mathrm{q}, J=7.2 \mathrm{~Hz}, 2 \mathrm{H}), 3.65(\mathrm{~s}$, $2 \mathrm{H}), 1.33(\mathrm{t}, J=7.2 \mathrm{~Hz}, 3 \mathrm{H}), 1.29(\mathrm{t}, J=7.2 \mathrm{~Hz}, 3 \mathrm{H}) ;{ }^{13} \mathrm{C} \mathrm{NMR}\left(75 \mathrm{MHz}, \mathrm{CDCl}_{3}\right) \delta: 170.4,166.7$, 147.0, 138.0, 133.5, 132.3, 129.4, 127.6, 120.1, 116.2, 61.3, 60.5, 29.9, 14.4, 14.2; HRMS (ESI) $\mathrm{m} / \mathrm{z}:[\mathrm{M}+\mathrm{H}]^{+}$Calcd for $\mathrm{C}_{18} \mathrm{H}_{20} \mathrm{~N}_{2} \mathrm{O}_{4}{ }^{35} \mathrm{Cl}$ 363.1106; Found 363.1104.

One of aromatic carbons overlapped with other aromatic carbons in ${ }^{13} \mathrm{C}$ NMR spectrum.<smiles>CCOCCc1cn(-c2ccc(Br)cc2)nc1C=CCC(=O)OCC</smiles>

(E)-3-[1-(4-Bromophenyl)-4-(2-ethoxy-2-oxoethyl)-1H-pyrazol-3-yl]-2-propenoic acid ethyl ester (2h) $32.4 \mathrm{mg}$, 80\% yield; Reaction time: $4 \mathrm{~h}$; Purification by preparative TLC ( $n$-hexane: EtOAc $=2: 1)$; A yellow solid; Mp: 90-91 ${ }^{\circ} \mathrm{C}(n$-hexane-Et $2 \mathrm{O})$; IR (neat): 1736, $1710 \mathrm{~cm}^{-1} ;{ }^{1} \mathrm{H}$ NMR $\left(300 \mathrm{MHz}, \mathrm{CDCl}_{3}\right) \delta: 7.95(\mathrm{~s}, 1 \mathrm{H}), 7.63(\mathrm{~d}, J=16.2 \mathrm{~Hz}, 1 \mathrm{H}), 7.59-7.52(\mathrm{~m}, 4 \mathrm{H}), 6.66(\mathrm{~d}, J=16.2 \mathrm{~Hz}$, $1 \mathrm{H}), 4.26$ (q, $J=7.2 \mathrm{~Hz}, 2 \mathrm{H}), 4.19$ (q, $J=7.2 \mathrm{~Hz}, 2 \mathrm{H}), 3.64$ (s, 2H), 1.33 (t, $J=7.2 \mathrm{~Hz}, 3 \mathrm{H}), 1.29$ (t, $J=7.2 \mathrm{~Hz}, 3 \mathrm{H}) ;{ }^{13} \mathrm{C} \mathrm{NMR}\left(75 \mathrm{MHz}, \mathrm{CDCl}_{3}\right) \delta: 170.4,166.7,147.1,138.5,133.5,132.4,127.6$, 120.4, 120.1, 116.3, 61.3, 60.6, 29.9, 14.4, 14.3; HRMS (ESI) m/z: $[\mathrm{M}+\mathrm{H}]^{+}$Calcd for $\mathrm{C}_{18} \mathrm{H}_{20} \mathrm{~N}_{2} \mathrm{O}_{4}{ }^{79} \mathrm{Br}$ 407.0601; Found 407.0602.

One of aromatic carbons overlapped with other aromatic carbons in ${ }^{13} \mathrm{C} N \mathrm{NR}$ spectrum.

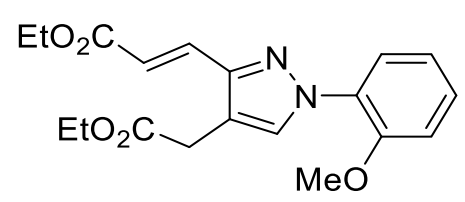

(E)-3-[4-(2-Ethoxy-2-oxoethyl)-1-(2-methoxyphenyl)-1 $H$-pyrazol-3-yl]-2-propenoic acid ethyl ester (2i) $8.2 \mathrm{mg}, 23 \%$ yield; Reaction time: $72 \mathrm{~h}$; Purification by preparative TLC ( $n$-hexane: EtOAc =2:1); A yellow oil; IR (neat): 1738, $1712 \mathrm{~cm}^{-1} ;{ }^{1} \mathrm{H}$ NMR $\left(300 \mathrm{MHz}, \mathrm{CDCl}_{3}\right) \delta: 8.06(\mathrm{~s}, 1 \mathrm{H})$, 7.75-7.72 (d, $J=8.0 \mathrm{~Hz}, 1 \mathrm{H}), 7.68(\mathrm{~d}, J=16.2 \mathrm{~Hz}, 1 \mathrm{H}), 7.32-7.26(\mathrm{~m}, 1 \mathrm{H}), 7.07-7.01(\mathrm{~m}, 2 \mathrm{H}), 6.64$ (d, $J=15.9 \mathrm{~Hz}, 1 \mathrm{H}), 4.25$ (q, $J=7.2 \mathrm{~Hz}, 2 \mathrm{H}), 4.18$ (q, $J=7.2 \mathrm{~Hz}, 2 \mathrm{H}), 3.88(\mathrm{~s}, 3 \mathrm{H}), 3.66$ (s, 2H), $1.32(\mathrm{t}, J=7.2 \mathrm{~Hz}, 3 \mathrm{H}), 1.29(\mathrm{t}, J=7.2 \mathrm{~Hz}, 3 \mathrm{H}) ;{ }^{13} \mathrm{C} \mathrm{NMR}\left(75 \mathrm{MHz}, \mathrm{CDCl}_{3}\right) \delta: 170.7,167.0,151.0$, $145.8,134.2,132.7,128.3,125.0,121.2,119.5,114.5,112.2,61.2,60.4,56.0,30.1,14.4,14.3$; HRMS (ESI) m/z: [M+ H] $]^{+}$Calcd for $\mathrm{C}_{19} \mathrm{H}_{23} \mathrm{~N}_{2} \mathrm{O}_{5}$ 359.1602; Found 359.1599.

One of aromatic carbons overlapped with other aromatic carbons in ${ }^{13} \mathrm{C}$ NMR spectrum. 
Scalable synthesis of pyrazoles $2 a(4.0 \mathrm{mmol}$ scale).<smiles>CCOC(=O)/C=C/C=N/Nc1ccccc1</smiles>

1a

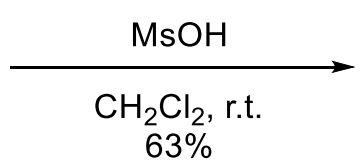

$63 \%$

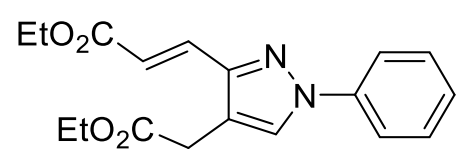

2a

To a solution of conjugated hydrazone $1 \mathrm{a}(1.00 \mathrm{~g}, 4.0 \mathrm{mmol})$ in $\mathrm{CH}_{2} \mathrm{Cl}_{2}(20 \mathrm{~mL})$ was added $\mathrm{MsOH}$ (261 $\mu \mathrm{L}, 4.0 \mathrm{mmol})$ at room temperature. After being stirred at the same temperature for $1 \mathrm{~h}$, the reaction mixture was quenched with saturated aqueous $\mathrm{NaHCO}_{3}(10 \mathrm{~mL})$ and extracted with $\mathrm{CHCl}_{3}$ $(80 \mathrm{~mL} \times 3)$. The organic phase was dried over $\mathrm{MgSO}_{4}$ and concentrated under reduced pressure. The crude product was purified by flash column chromatography ( $n$-hexane: EtOAc $=2: 1)$ to afford pyrazole $2 \mathrm{a}(455.2 \mathrm{mg}, 63 \%)$ as a yellow oil.

\subsection{One-pot synthesis of pyrazole $2 a$.}

1) $\mathrm{MeO}$<smiles>Cc1ccc(NN)cc1</smiles><smiles>CCOC(=O)CC=CC=O</smiles>
3<smiles>CCOC(=O)C=Cc1nn(-c2ccc(OC)cc2)cc1CC=CCOC</smiles>

2a

To a solution of ethyl trans-4-oxo-2-butenoate $3(47 \mu \mathrm{L}, 0.39 \mathrm{mmol})$ in $\mathrm{CH}_{2} \mathrm{Cl}_{2}(10 \mathrm{~mL})$ were added 4-methoxyphenylhydrazine hydrochloride $(68 \mathrm{mg}, 0.39 \mathrm{mmol})$ and 2,4,6-collidine $(52 \mu \mathrm{L}, 0.39$ $\mathrm{mmol})$ at room temperature. After being stirred at the same temperature for $2 \mathrm{~h}, \mathrm{MsOH}(51 \mu \mathrm{L}, 0.78$ mmol) was added. The reaction mixture quenched with saturated aqueous $\mathrm{NaHCO}_{3}(5 \mathrm{~mL})$ and extracted with $\mathrm{CHCl}_{3}(30 \mathrm{~mL} \times 3)$. The organic phase was dried over $\mathrm{MgSO}_{4}$ and concentrated under reduced pressure. The crude product was purified by preparative TLC $(n$-hexane:EtOAc $=2: 1)$ to afford the pyrazole $\mathbf{2 a}$ ( $45.4 \mathrm{mg}, 65 \%$ yield).

\subsection{General procedure for synthesis of pyrazoles 5 (cross-condensation-I).}

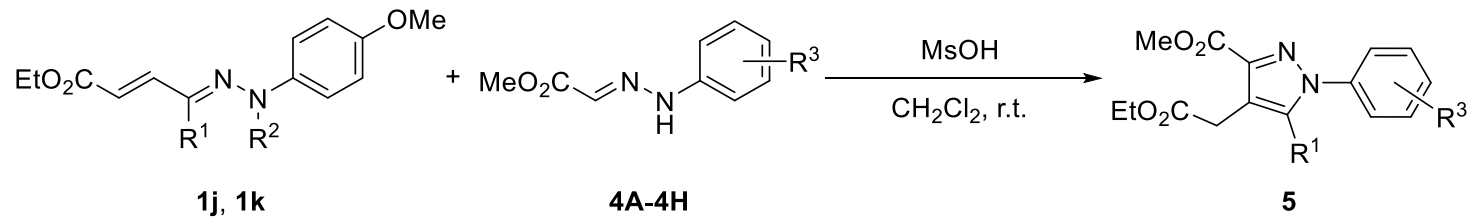

To a solution of conjugated hydrazones $\mathbf{1 j}$ or $\mathbf{1} \mathbf{k}(0.20 \mathrm{mmol})$ and hydrazones $\mathbf{4 A}-\mathbf{H}(0.24 \mathrm{mmol})$ in $\mathrm{CH}_{2} \mathrm{Cl}_{2}(2.0 \mathrm{~mL})$ was added $\mathrm{MsOH}(26 \mu \mathrm{L}, 0.40 \mathrm{mmol})$ at room temperature. After being stirred at 
the same temperature for several hours, the reaction mixture was quenched with saturated aqueous $\mathrm{NaHCO}_{3}(5 \mathrm{~mL})$ and extracted with $\mathrm{CHCl}_{3}(30 \mathrm{~mL} \times 3)$. The organic phase was dried over $\mathrm{MgSO}_{4}$ and concentrated under reduced pressure. The crude product was purified by preparative TLC ( $n$-hexane:AcOEt) to afford the corresponding pyrazoles $\mathbf{5} \mathbf{j} \mathbf{A}-\mathbf{j} \mathbf{H}, \mathbf{k A}$.<smiles>CCOCc1cn(-c2ccc(OC)cc2)nc1C(OC)OC</smiles>

[3-(Methoxycarbonyl)-1-(4-methoxyphenyl)-1H-pyrazol-4-yl]acetic acid ethyl ester (5jA) 60.3 mg, 95\% yield; Reaction time: $4 \mathrm{~h}$; Purification by preparative TLC ( $n$-hexane: EtOAc $=2: 1$ ); A yellow solid; Mp: $65-67{ }^{\circ} \mathrm{C}$ ( $n$-hexane-Et $2 \mathrm{O}$ ); IR (neat): $1727 \mathrm{~cm}^{-1} ;{ }^{1} \mathrm{H}$ NMR $\left(300 \mathrm{MHz}, \mathrm{CDCl}_{3}\right) \delta$ : 7.90 (s, 1H), 7.60 (d, $J=9.0 \mathrm{~Hz}, 2 \mathrm{H}), 6.94$ (d, $J=9.0 \mathrm{~Hz}, 2 \mathrm{H}), 4.19$ (q, $J=7.2 \mathrm{~Hz}, 2 \mathrm{H}), 3.93$ (s, 3H), 3.89 (s, 2H), 3.83 (s, 3H), 1.29 (t, $J=7.2 \mathrm{~Hz}, 3 \mathrm{H}) ;{ }^{13} \mathrm{C} \mathrm{NMR}\left(75 \mathrm{MHz}, \mathrm{CDCl}_{3}\right) \delta: 170.9,162.7$, 158.8, 141.4, 132.9, 128.6, 121.4, 118.6, 114.3, 60.8, 55.5, 51.8, 30.1, 14.2; HRMS (ESI) m/z: [M + $\mathrm{H}]^{+}$Calcd for $\mathrm{C}_{16} \mathrm{H}_{19} \mathrm{~N}_{2} \mathrm{O}_{5} 319.1289$; Found 319.1285 .<smiles>CCOC(=O)Cc1cn(-c2ccc(C)cc2)nc1C(C)=O</smiles>

[3-(Methoxycarbonyl)-1-(4-methylphenyl)-1 H-pyrazol-4-yl]acetic acid ethyl ester (5jB) 48.9 mg, $81 \%$ yield; Reaction time: $4 \mathrm{~h}$; Purification by preparative TLC ( $n$-hexane: EtOAc $=2: 1)$; A yellow oil; IR (neat): 1736, $1719 \mathrm{~cm}^{-1} ;{ }^{1} \mathrm{H}$ NMR (300 MHz, $\left.\mathrm{CDCl}_{3}\right) \delta: 7.95$ (s, 1H), 7.58 (d, $J=8.4 \mathrm{~Hz}, 2 \mathrm{H}$ ), $6.23(\mathrm{~d}, J=7.2 \mathrm{~Hz}, 2 \mathrm{H}), 4.19$ (q, $J=7.2 \mathrm{~Hz}, 2 \mathrm{H}), 3.93(\mathrm{~s}, 3 \mathrm{H}), 3.89$ (s, 2H), 2.38 (s, 3H), 1.29 (t, $J=$ $7.2 \mathrm{~Hz}, 3 \mathrm{H}) ;{ }^{13} \mathrm{C}$ NMR $\left(75 \mathrm{MHz}, \mathrm{CDCl}_{3}\right) \delta: 170.9,162.8,141.6,137.4,137.1,129.8,128.5,119.7$, 118.7, 60.9, 51.9, 30.2, 21.0, 14.3; HRMS (ESI) m/z: [M + Na $]^{+}$Calcd for $\mathrm{C}_{16} \mathrm{H}_{18} \mathrm{~N}_{2} \mathrm{O}_{4} \mathrm{Na} 325.1159$; Found 325.1156.<smiles>CCOC(=O)Cc1cn(-c2ccc(C(C)(C)C)cc2)nc1C(C)=O</smiles>

[1-[4-(1,1-Dimethylethyl)phenyl]-3-(methoxycarbonyl)-1 $H$-pyrazol-4-yl]acetic acid ethyl ester (5jC) $73.5 \mathrm{mg}$, 99\% yield; Reaction time: $6 \mathrm{~h}$; Purification by preparative TLC ( $n$-hexane: EtOAc $=$ 2:1); A yellow oil; IR (neat): 1739, $1722 \mathrm{~cm}^{-1} ;{ }^{1} \mathrm{H}$ NMR (300 MHz, $\left.\mathrm{CDCl}_{3}\right) \delta: 7.96(\mathrm{~s}, 1 \mathrm{H}), 7.61$ (d, $J=8.7 \mathrm{~Hz}, 2 \mathrm{H}$ ), 7.44 (d, $J=9.0 \mathrm{~Hz}, 2 \mathrm{H}), 4.19$ (q, $J=7.2 \mathrm{~Hz}, 2 \mathrm{H}), 3.93$ (s, 3H), 3.89 (s, 2H), 1.34 (s, 9H), 1.29 (t, $J=7.2 \mathrm{~Hz}, 3 \mathrm{H}) ;{ }^{13} \mathrm{C} \mathrm{NMR}\left(75 \mathrm{MHz}, \mathrm{CDCl}_{3}\right) \delta: 170.9,162.8,150.7,141.7,136.9,128.5$, 126.1, 119.5, 118.7, 60.9, 51.9, 34.7, 31.3, 30.2, 14.3; HRMS (ESI) m/z: $[\mathrm{M}+\mathrm{Na}]^{+}$Calcd for $\mathrm{C}_{19} \mathrm{H}_{24} \mathrm{~N}_{2} \mathrm{O}_{4} \mathrm{Na} 367.1628$; Found 367.1627. 


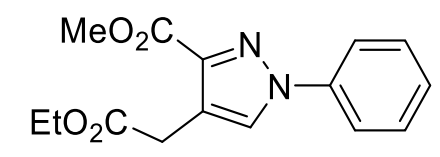

[3-(Methoxycarbonyl)-1-phenyl-1H-pyrazol-4-yl]acetic acid ethyl ester (5jD) $45.3 \mathrm{mg}, 79 \%$ yield; Reaction time: 6 h; Purification by preparative TLC ( $n$-hexane: EtOAc $=2: 1$ ); A yellow oil; IR (neat): $1734 \mathrm{~cm}^{-1} ;{ }^{1} \mathrm{H}$ NMR $\left(300 \mathrm{MHz}, \mathrm{CDCl}_{3}\right) \delta: 8.00(\mathrm{~s}, 1 \mathrm{H}), 7.69(\mathrm{~d}, J=8.7 \mathrm{~Hz}, 2 \mathrm{H}), 7.42(\mathrm{t}, J=$ $8.4 \mathrm{~Hz}, 2 \mathrm{H}), 7.31(\mathrm{t}, J=8.4 \mathrm{~Hz}, 1 \mathrm{H}), 4.19(\mathrm{q}, J=7.2 \mathrm{~Hz}, 2 \mathrm{H}), 3.93(\mathrm{~s}, 3 \mathrm{H}), 3.89(\mathrm{~s}, 2 \mathrm{H}), 1.28(\mathrm{t}, J=$ $7.2 \mathrm{~Hz}, 3 \mathrm{H}) ;{ }^{13} \mathrm{C} \mathrm{NMR}\left(75 \mathrm{MHz}, \mathrm{CDCl}_{3}\right) \delta: 170.8,162.7,141.8,139.2,129.2,128.5,127.3,119.6$, 118.8, 60.7, 51.7, 29.9, 14.0; HRMS (ESI) m/z: $[\mathrm{M}+\mathrm{H}]^{+}$Calcd for $\mathrm{C}_{15} \mathrm{H}_{17} \mathrm{~N}_{2} \mathrm{O}_{4}$ 289.1183; Found 289.1181 .

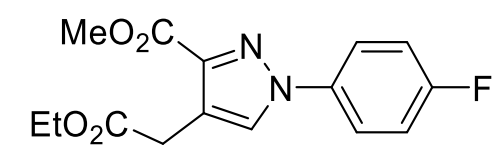

[1-(4-Fluorophenyl)-3-(methoxycarbonyl)-1H-pyrazol-4-yl]acetic acid ethyl ester (5jE) $\mathbf{1 j}$ (131 $\mathrm{mg}, 0.50 \mathrm{mmol})$ and $4 \mathbf{E}(118 \mathrm{mg}, 0.60 \mathrm{mmol})$ were used. $129.8 \mathrm{mg}, 85 \%$ yield; Reaction time: $5 \mathrm{~h}$; Purification by preparative TLC ( $n$-hexane: EtOAc $=2: 1)$; Yellow crystals; $\mathrm{Mp}: 79-81{ }^{\circ} \mathrm{C}$ (n-hexane-Et $2 \mathrm{O}$ ); IR (neat): $1733 \mathrm{~cm}^{-1} ;{ }^{1} \mathrm{H} \mathrm{NMR}\left(300 \mathrm{MHz}, \mathrm{CDCl}_{3}\right) \delta: 7.94(\mathrm{~s}, 1 \mathrm{H}), 7.62(\mathrm{dd}, J=9.1$, $4.6 \mathrm{~Hz}, 2 \mathrm{H}), 7.14$ (dd, $J=9.1,8.0 \mathrm{~Hz}, 2 \mathrm{H}), 4.20$ (q, $J=7.2 \mathrm{~Hz}, 2 \mathrm{H}), 3.94$ (s, 3H), 3.90 (s, 2H), 1.29 $(\mathrm{t}, J=7.2 \mathrm{~Hz}, 3 \mathrm{H}) ;{ }^{13} \mathrm{C} \mathrm{NMR}\left(150 \mathrm{MHz}, \mathrm{CDCl}_{3}\right) \delta: 171.0,162.8,162.6\left(\mathrm{C}-\mathrm{F},{ }^{1} J_{\mathrm{C}-\mathrm{F}}=246.0 \mathrm{~Hz}\right)$, $161.0\left(\mathrm{C}-\mathrm{F},{ }^{1} J_{\mathrm{C}-\mathrm{F}}=246.0 \mathrm{~Hz}\right), 142.2,135.8\left(\mathrm{C}-\mathrm{F},{ }^{4} J_{\mathrm{C}-\mathrm{F}}=3.0 \mathrm{~Hz}\right), 135.8\left(\mathrm{C}-\mathrm{F},{ }^{4} J_{\mathrm{C}-\mathrm{F}}=3.0 \mathrm{~Hz}\right), 128.8$, $121.9\left(\mathrm{C}-\mathrm{F},{ }^{3} J_{\mathrm{C}-\mathrm{F}}=8.4 \mathrm{~Hz}\right), 121.9\left(\mathrm{C}-\mathrm{F},{ }^{3} J_{\mathrm{C}-\mathrm{F}}=8.4 \mathrm{~Hz}\right), 119.1,116.4\left(\mathrm{C}-\mathrm{F},{ }^{2} J_{\mathrm{C}-\mathrm{F}}=23.0 \mathrm{~Hz}\right), 116.2$ $\left(\mathrm{C}-\mathrm{F},{ }^{2} J_{\mathrm{C}-\mathrm{F}}=23.0 \mathrm{~Hz}\right), 61.0,52.0,30.1,14.2$; HRMS (ESI) $\mathrm{m} / \mathrm{z}:[\mathrm{M}+\mathrm{H}]^{+}$Calcd for $\mathrm{C}_{15} \mathrm{H}_{16} \mathrm{~N}_{2} \mathrm{O}_{4} \mathrm{~F}$ 307.1089; Found 307.1088.<smiles>CCOC(=O)c1cn(-c2ccc(Cl)cc2)nc1C(C)=O</smiles>

[1-(4-Chlorophenyl)-3-(methoxycarbonyl)-1 H-pyrazol-4-yl]acetic acid ethyl ester (5jF) $56.2 \mathrm{mg}$, $87 \%$ yield; Reaction time: $6 \mathrm{~h}$; Purification by preparative TLC ( $n$-hexane: EtOAc $=2: 1$ ); Yellow crystals; Mp: $62-66{ }^{\circ} \mathrm{C}\left(n\right.$-hexane-Et 2 O); IR (neat): $1735 \mathrm{~cm}^{-1} ;{ }^{1} \mathrm{H}$ NMR $\left(300 \mathrm{MHz}, \mathrm{CDCl}_{3}\right) \delta: 7.98$ $(\mathrm{s}, 1 \mathrm{H}), 7.66(\mathrm{~d}, J=9.0 \mathrm{~Hz}, 2 \mathrm{H}), 7.41(\mathrm{~d}, J=9.0 \mathrm{~Hz}, 2 \mathrm{H}), 4.19(\mathrm{q}, J=7.2 \mathrm{~Hz}, 2 \mathrm{H}), 3.94(\mathrm{~s}, 3 \mathrm{H}), 3.89$ (s, 2H), 1.29 (t, $J=7.2 \mathrm{~Hz}, 3 \mathrm{H}) ;{ }^{13} \mathrm{C}$ NMR $\left(75 \mathrm{MHz}, \mathrm{CDCl}_{3}\right) \delta: 170.8,162.6,142.2,137.8,133.1$, 129.4, 128.5, 121.0, 119.2, 61.0, 52.1, 30.2, 14.3; HRMS (ESI) $\mathrm{m} / \mathrm{z}:[\mathrm{M}+\mathrm{H}]^{+}$Calcd for $\mathrm{C}_{15} \mathrm{H}_{16} \mathrm{~N}_{2} \mathrm{O}_{4}{ }^{35} \mathrm{Cl} 323.0793$; Found 323.0792. 
<smiles>CCOC(=O)Cc1cn(-c2ccc(Br)cc2)nc1COC</smiles>

[1-(4-Bromophenyl)-3-(methoxycarbonyl)-1 $H$-pyrazol-4-yl]acetic acid ethyl ester (5jG) $51.0 \mathrm{mg}$, $69 \%$ yield; Reaction time: 6 h; Purification by preparative TLC $(n$-hexane: EtOAc $=2: 1)$; A yellow oil; IR (neat): $1729 \mathrm{~cm}^{-1} ;{ }^{1} \mathrm{H}$ NMR (300 MHz, $\left.\mathrm{CDCl}_{3}\right) \delta: 7.99$ (s, 1H), 7.62-7.54 (m, 4H), 4.19 (q, $J$ $=7.2 \mathrm{~Hz}, 2 \mathrm{H}), 3.94(\mathrm{~s}, 3 \mathrm{H}), 3.89(\mathrm{~s}, 2 \mathrm{H}), 1.29$ (t, $J=7.2 \mathrm{~Hz}, 3 \mathrm{H}) ;{ }^{13} \mathrm{C}$ NMR $\left(75 \mathrm{MHz}, \mathrm{CDCl}_{3}\right) \delta$ : 170.7, 162.5, 142.3, 138.3, 132.4, 128.4, 121.2, 120.9, 119.2, 61.0, 52.0, 30.2, 14.3; HRMS (ESI) $\mathrm{m} / \mathrm{z}:[\mathrm{M}+\mathrm{Na}]^{+}$Calcd for $\mathrm{C}_{15} \mathrm{H}_{15} \mathrm{~N}_{2} \mathrm{O}_{4}{ }^{79} \mathrm{BrNa} 389.0107$; Found 389.0106 .

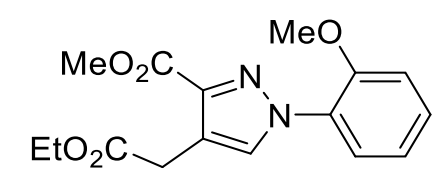

[3-(Methoxycarbonyl)-1-(2-methoxyphenyl)-1H-pyrazol-4-yl]acetic acid ethyl ester (5jH) 38.4 mg, 60\%; Reaction time: 6 h; Purification by preparative TLC ( $n$-hexane: EtOAc $=2: 1$ ); A yellow oil; IR (neat): 1736, $1719 \mathrm{~cm}^{-1}$; ${ }^{1} \mathrm{H}$ NMR $\left(300 \mathrm{MHz}, \mathrm{CDCl}_{3}\right) \delta: 8.02(\mathrm{~s}, 1 \mathrm{H}), 7.70(\mathrm{~d}, J=7.8 \mathrm{~Hz}, 1 \mathrm{H})$, 7.32 (t, $J=7.8 \mathrm{~Hz}, 1 \mathrm{H}), 7.06-7.00(\mathrm{~m}, 2 \mathrm{H}), 4.19$ (q, $J=7.2 \mathrm{~Hz}, 2 \mathrm{H}), 3.92(\mathrm{~s}, 3 \mathrm{H}), 3.90(\mathrm{~s}, 2 \mathrm{H}), 3.86$ $(\mathrm{s}, 3 \mathrm{H}), 1.29$ (t, $J=7.2 \mathrm{~Hz}, 3 \mathrm{H}) ;{ }^{13} \mathrm{C}$ NMR $\left(75 \mathrm{MHz}, \mathrm{CDCl}_{3}\right) \delta: 171.0,162.9,151.5,141.0,133.2$, 129.0, 125.8, 121.0, 117.4, 112.0, 60.8, 55.9, 51.8, 30.3, 14.3; HRMS (ESI) m/z: $[\mathrm{M}+\mathrm{H}]^{+}$Calcd for $\mathrm{C}_{16} \mathrm{H}_{19} \mathrm{~N}_{2} \mathrm{O}_{5} 319.1289$; Found 319.1287.

One of aromatic carbons overlapped with other aromatic carbons in ${ }^{13} \mathrm{C}$ NMR spectrum.<smiles>COCc1c(C(=O)OC)nn(-c2ccc(OC)cc2)c1C</smiles>

[3-(Methoxycarbonyl)-1-(4-methoxyphenyl)-5-methyl-1H-pyrazol-4-yl]acetic acid methyl ester (5kA) $15.3 \mathrm{mg}, 24 \%$ yield; Reaction time: $5 \mathrm{~h}$; Purification by preparative TLC ( $n$-hexane: EtOAc $=$ 2:1); An orange oil; IR (neat): 1739, $1717 \mathrm{~cm}^{-1} ;{ }^{1} \mathrm{H}$ NMR (300 MHz, $\left.\mathrm{CDCl}_{3}\right) \delta: 7.37$ (d, $J=9.0 \mathrm{~Hz}$, $2 \mathrm{H}), 6.97(\mathrm{~d}, J=9.0 \mathrm{~Hz}, 2 \mathrm{H}), 3.91(\mathrm{~s}, 3 \mathrm{H}), 3.86(\mathrm{~s}, 3 \mathrm{H}), 3.83(\mathrm{~s}, 2 \mathrm{H}), 3.73(\mathrm{~s}, 3 \mathrm{H}), 2.22(\mathrm{~s}, 3 \mathrm{H}) ;{ }^{13} \mathrm{C}$ NMR (75 MHz, $\left.\mathrm{CDCl}_{3}\right) \delta: 171.6,163.3,159.7,140.9,139.6,132.1,127.0,115.2,114.2,55.5,52.0$, 51.8, 29.6, 10.6; HRMS (ESI) m/z: [M+ H] $]^{+}$Calcd for $\mathrm{C}_{16} \mathrm{H}_{19} \mathrm{~N}_{2} \mathrm{O}_{5}$ 319.1289; Found 319.1287. 
2.6 General procedure for synthesis of pyrazoles 5 (cross-condensation-II).

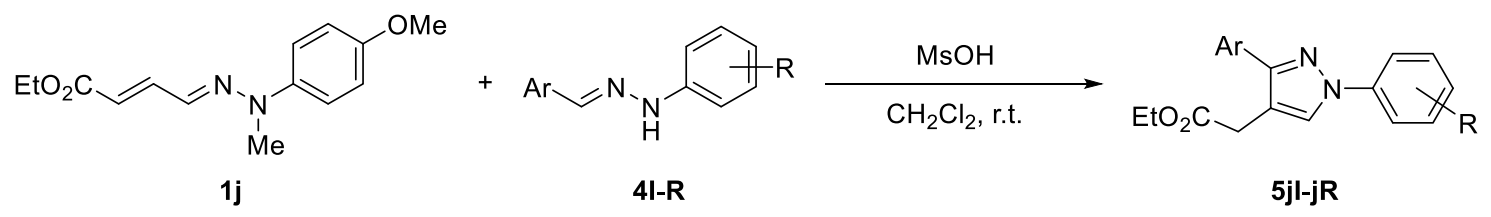

To a solution of conjugated hydrazone $\mathbf{1 j} \mathbf{j}(26.2 \mathrm{mg}, 0.10 \mathrm{mmol})$ and hydrazones $\mathbf{4 I - R}(0.20 \mathrm{mmol})$ in $\mathrm{CH}_{2} \mathrm{Cl}_{2}(2.0 \mathrm{~mL})$ was added $\mathrm{MsOH}(32.4 \mu \mathrm{L}, 0.50 \mathrm{mmol})$ at room temperature. After being stirred at the same temperature for several hours, the reaction mixture was quenched with saturated aqueous $\mathrm{NaHCO}_{3}(5 \mathrm{~mL})$ and extracted with $\mathrm{CHCl}_{3}(30 \mathrm{~mL} \times 3)$. The organic phase was dried over $\mathrm{MgSO}_{4}$ and concentrated under reduced pressure. The crude product was purified by preparative TLC ( $n$-hexane:AcOEt $=3: 1)$ to afford the corresponding pyrazoles $\mathbf{5 j I - j R}$.<smiles>CCOCc1cn(-c2ccc(OC)cc2)nc1-c1ccccc1</smiles>

[1-(4-Methoxyphenyl)-3-phenyl-1H-pyrazol-4-yl]acetic acid ethyl ester (5jI) 27.9 mg, 83\% yield; Reaction time: 2 h; Purification by preparative TLC ( $n$-hexane: EtOAc $=2: 1$ ); An orange oil; IR (neat): $1733 \mathrm{~cm}^{-1} ;{ }^{1} \mathrm{H}$ NMR (300 MHz, $\left.\mathrm{CDCl}_{3}\right) \delta: 7.93(\mathrm{~s}, 1 \mathrm{H}), 7.67-7.61(\mathrm{~m}, 4 \mathrm{H}), 7.45-7.31(\mathrm{~m}, 3 \mathrm{H})$, $6.95(\mathrm{~d}, J=9.0 \mathrm{~Hz}, 2 \mathrm{H}), 4.16$ (q, $J=7.2 \mathrm{~Hz}, 2 \mathrm{H}), 3.83(\mathrm{~s}, 3 \mathrm{H}), 3.68$ (s, 2H), 1.24 (t, $J=7.2 \mathrm{~Hz}, 3 \mathrm{H})$; ${ }^{13} \mathrm{C}$ NMR $\left(75 \mathrm{MHz}, \mathrm{CDCl}_{3}\right) \delta: 171.4,158.0,151.5,133.7,133.1,128.4,128.0,127.8,120.6,114.4$, 112.7, 61.0, 55.6, 30.6, 14.3; HRMS (ESI) m/z: $[\mathrm{M}+\mathrm{H}]^{+}$Calcd for $\mathrm{C}_{20} \mathrm{H}_{21} \mathrm{~N}_{2} \mathrm{O}_{3}$ 337.1547; Found 337.1548 .

One of aromatic carbons overlapped with other aromatic carbons in ${ }^{13} \mathrm{C}$ NMR spectrum.<smiles>CCOC(=O)Cc1cn(-c2ccc(OC)cc2)nc1-c1ccc(OC)cc1</smiles>

[1,3-Bis(4-methoxyphenyl)-1 H-pyrazol-4-yl]acetic acid ethyl ester (5jJ) $19.3 \mathrm{mg}, 53 \%$ yield; Reaction time: $2 \mathrm{~h}$; Purification by preparative TLC ( $n$-hexane: EtOAc $=2: 1)$; An orange oil; IR (neat): $1736 \mathrm{~cm}^{-1} ;{ }^{1} \mathrm{H}$ NMR $\left(300 \mathrm{MHz}, \mathrm{CDCl}_{3}\right) \delta: 7.93(\mathrm{~s}, 1 \mathrm{H}), 7.64(\mathrm{~d}, J=9.3 \mathrm{~Hz}, 2 \mathrm{H}), 7.61(\mathrm{~d}, J=$ $8.7 \mathrm{~Hz}, 2 \mathrm{H}), 6.98(\mathrm{~d}, J=9.0 \mathrm{~Hz}, 2 \mathrm{H}), 6.96(\mathrm{~d}, J=9.3 \mathrm{~Hz}, 2 \mathrm{H}), 4.17$ (q, $J=7.2 \mathrm{~Hz}, 2 \mathrm{H}), 3.85(\mathrm{~s}, 3 \mathrm{H})$, $3.84(\mathrm{~s}, 3 \mathrm{H}), 3.67(\mathrm{~s}, 2 \mathrm{H}), 1.25(\mathrm{t}, J=7.2 \mathrm{~Hz}, 3 \mathrm{H}) ;{ }^{13} \mathrm{C} \mathrm{NMR}\left(75 \mathrm{MHz}, \mathrm{CDCl}_{3}\right) \delta: 171.6,159.4$, $158.1,151.4,133.9,129.3,127.7,125.7,120.5,114.4,114.0,112.5,61.0,55.6,55.3,30.6,14.2$; HRMS (ESI) m/z: [M+ H] $]^{+}$Calcd for $\mathrm{C}_{21} \mathrm{H}_{23} \mathrm{~N}_{2} \mathrm{O}_{4}$ 367.1652; Found 367.1655. 
<smiles>CCOC(=O)Cc1cn(-c2ccc(OC)cc2)nc1-c1ccc(C)cc1</smiles>

[1-(4-Methoxyphenyl)-3-(4-methylphenyl)-1 H-pyrazol-4-yl]acetic acid ethyl ester (5jK) $20.7 \mathrm{mg}$, $59 \%$ yield; Reaction time: $4 \mathrm{~h}$; Purification by preparative TLC ( $n$-hexane: EtOAc $=2: 1)$; An orange oil; IR (neat): $1736 \mathrm{~cm}^{-1} ;{ }^{1} \mathrm{H}$ NMR (300 MHz, $\left.\mathrm{CDCl}_{3}\right) \delta: 7.91(\mathrm{~s}, 1 \mathrm{H}), 7.61(\mathrm{~d}, J=8.7 \mathrm{~Hz}, 2 \mathrm{H}), 7.54$ (d, $J=7.8 \mathrm{~Hz}, 2 \mathrm{H}), 7.22$ (d, $J=7.8 \mathrm{~Hz}, 2 \mathrm{H}), 6.94$ (d, $J=8.7 \mathrm{~Hz}, 2 \mathrm{H}), 4.15$ (q, $J=7.2 \mathrm{~Hz}, 2 \mathrm{H}), 3.82$ (s, 3H), $3.66(\mathrm{~s}, 2 \mathrm{H}), 2.38(\mathrm{~s}, 3 \mathrm{H}), 1.24(\mathrm{t}, J=7.2 \mathrm{~Hz}, 3 \mathrm{H}) ;{ }^{13} \mathrm{C} \mathrm{NMR}\left(75 \mathrm{MHz}, \mathrm{CDCl}_{3}\right) \delta: 171.2$, $157.8,151.3,137.4,133.6,130.0,129.0,127.8,127.5,120.4$, 114.3, 112.5, 61.0, 55.6, 30.7, 21.5, 14.4; HRMS (ESI) $\mathrm{m} / \mathrm{z}$ : [M+ Na $]^{+}$Calcd for $\mathrm{C}_{21} \mathrm{H}_{22} \mathrm{~N}_{2} \mathrm{O}_{3} \mathrm{Na}$ 373.1523; Found 373.1526.

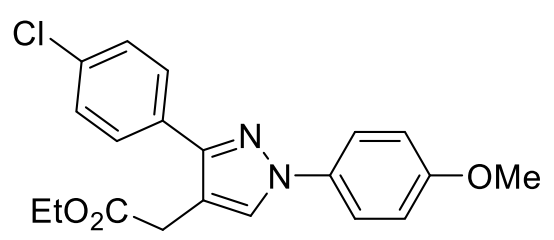

[3-(4-Chlorophenyl)-1-(4-methoxyphenyl)-1H-pyrazol-4-yl]acetic acid ethyl ester (5jL) $26.7 \mathrm{mg}$, $72 \%$ yield; Reaction time: $2 \mathrm{~h}$; Purification by preparative TLC ( $n$-hexane: EtOAc $=2: 1)$; An orange oil; IR (neat): $1736 \mathrm{~cm}^{-1}$; ${ }^{1} \mathrm{H}$ NMR $\left(300 \mathrm{MHz}, \mathrm{CDCl}_{3}\right) \delta: 7.91(\mathrm{~s}, 1 \mathrm{H}), 7.61(\mathrm{~d}, J=8.4 \mathrm{~Hz}$, 2H), 7.60 (d, $J=9.0 \mathrm{~Hz}, 2 \mathrm{H}), 7.39$ (d, $J=8.4 \mathrm{~Hz}, 2 \mathrm{H}), 6.94$ (d, $J=9.0 \mathrm{~Hz}, 2 \mathrm{H}), 4.16$ (q, $J=7.2 \mathrm{~Hz}$, $2 \mathrm{H}), 3.83(\mathrm{~s}, 3 \mathrm{H}), 3.65(\mathrm{~s}, 2 \mathrm{H}), 1.25(\mathrm{t}, J=7.2 \mathrm{~Hz}, 3 \mathrm{H}) ;{ }^{13} \mathrm{C} \mathrm{NMR}\left(75 \mathrm{MHz}, \mathrm{CDCl}_{3}\right) \delta: 171.0,158.0$, 150.1, 133.6, 133.4, 131.5, 129.1, 128.5, 127.9, 120.5, 114.3, 112.6, 61.1, 55.6, 30.6, 14.4; HRMS (ESI) $\mathrm{m} / \mathrm{z}:[\mathrm{M}+\mathrm{H}]^{+}$Calcd for $\mathrm{C}_{20} \mathrm{H}_{20} \mathrm{~N}_{2} \mathrm{O}_{3}{ }^{35} \mathrm{Cl}$ 371.1157; Found 371.1161 .

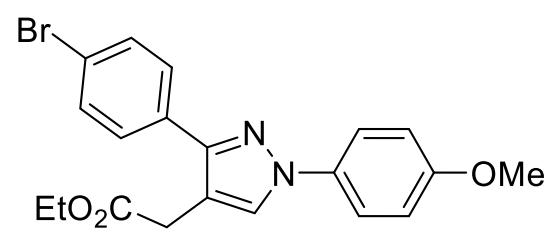

[3-(4-Bromophenyl)-1-(4-methoxyphenyl)-1 $H$-pyrazol-4-yl]acetic acid ethyl ester (5jM) $24.0 \mathrm{mg}$, $58 \%$ yield; Reaction time: $2 \mathrm{~h}$; Purification by preparative TLC ( $n$-hexane: EtOAc $=2: 1$ ); An orange oil; IR (neat): $1736 \mathrm{~cm}^{-1} ;{ }^{1} \mathrm{H}$ NMR $\left(300 \mathrm{MHz}, \mathrm{CDCl}_{3}\right) \delta: 7.91$ (s, $\left.1 \mathrm{H}\right), 7.60$ (d, $\left.J=9.0 \mathrm{~Hz}, 2 \mathrm{H}\right), 7.55$ (m, 4H), 6.95 (d, $J=9.0 \mathrm{~Hz}, 2 \mathrm{H}), 4.16$ (q, $J=7.2 \mathrm{~Hz}, 2 \mathrm{H}), 3.83$ (s, 3H), 3.65 (s, 2H), 1.25 (t, $J=7.2$ $\mathrm{Hz}, 3 \mathrm{H}) ;{ }^{13} \mathrm{C}$ NMR $\left(75 \mathrm{MHz}, \mathrm{CDCl}_{3}\right) \delta: 170.0,158.0,150.1,133.4,131.9,131.4,129.4,127.9$, 121.9, 120.5, 114.3, 112.6, 61.2, 55.6, 30.6, 14.4; HRMS (ESI) m/z: $[\mathrm{M}+\mathrm{H}]^{+}$Calcd for $\mathrm{C}_{20} \mathrm{H}_{20} \mathrm{~N}_{2} \mathrm{O}_{3}{ }^{79} \mathrm{Br} 415.0652$; Found 415.0657 . 


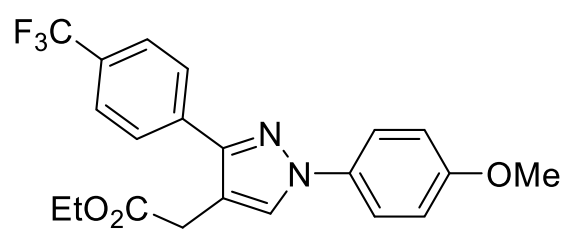

[1-(4-Methoxyphenyl)-3-[4-(trifluoromethyl)phenyl)]-1H-pyrazol-4-yl]acetic acid ethyl ester (5jN) $30.3 \mathrm{mg}$, 75\% yield; Reaction time: 3 h; Purification by preparative TLC ( $n$-hexane: EtOAc $=$ 2:1); An orange oil; IR (neat): $1736 \mathrm{~cm}^{-1} ;{ }^{1} \mathrm{H} \mathrm{NMR}\left(300 \mathrm{MHz}, \mathrm{CDCl}_{3}\right) \delta: 7.97$ (s, 1H), 7.83 (d, $J=$ $8.1 \mathrm{~Hz}, 2 \mathrm{H}), 7.70(\mathrm{~d}, J=8.1 \mathrm{~Hz}, 2 \mathrm{H}), 7.65(\mathrm{~d}, J=9.0 \mathrm{~Hz}, 2 \mathrm{H}), 6.98(\mathrm{~d}, J=9.0 \mathrm{~Hz}, 2 \mathrm{H}), 4.18$ (q, $J=$ $7.2 \mathrm{~Hz}, 2 \mathrm{H}), 3.85(\mathrm{~s}, 3 \mathrm{H}), 3.69(\mathrm{~s}, 2 \mathrm{H}), 1.25(\mathrm{t}, J=7.2 \mathrm{~Hz}, 3 \mathrm{H}) ;{ }^{13} \mathrm{C} \mathrm{NMR}\left(150 \mathrm{MHz}, \mathrm{CDCl}_{3}\right) \delta$ : 171.2, 158.4, 150.0, 136.8, 133.6, $130.0\left(\mathrm{C}-\mathrm{F},{ }^{2} J_{\mathrm{C}-\mathrm{F}}=32.2 \mathrm{~Hz}\right), 129.8\left(\mathrm{C}-\mathrm{F},{ }^{2} J_{\mathrm{C}-\mathrm{F}}=32.2 \mathrm{~Hz}\right), 129.6$ $\left(\mathrm{C}-\mathrm{F},{ }^{2} J_{\mathrm{C}-\mathrm{F}}=32.2 \mathrm{~Hz}\right), 129.4\left(\mathrm{C}-\mathrm{F},{ }^{2} J_{\mathrm{C}-\mathrm{F}}=32.2 \mathrm{~Hz}\right), 128.3,128.2,126.9\left(\mathrm{C}-\mathrm{F},{ }^{1} J_{\mathrm{C}-\mathrm{F}}=270.2 \mathrm{~Hz}\right)$, $125.5\left(\mathrm{C}-\mathrm{F},{ }^{3} J_{\mathrm{C}-\mathrm{F}}=3.7 \mathrm{~Hz}\right), 125.5\left(\mathrm{C}-\mathrm{F},{ }^{3} J_{\mathrm{C}-\mathrm{F}}=3.7 \mathrm{~Hz}\right), 125.5\left(\mathrm{C}-\mathrm{F},{ }^{3} J_{\mathrm{C}-\mathrm{F}}=3.7 \mathrm{~Hz}\right), 125.4\left(\mathrm{C}-\mathrm{F},{ }^{3} J_{\mathrm{C}-\mathrm{F}}\right.$ $=3.7 \mathrm{~Hz}), 125.1\left(\mathrm{C}-\mathrm{F},{ }^{1} J_{\mathrm{C}-\mathrm{F}}=270.2 \mathrm{~Hz}\right), 123.3\left(\mathrm{C}-\mathrm{F},{ }^{1} J_{\mathrm{C}-\mathrm{F}}=270.2 \mathrm{~Hz}\right), 121.5\left(\mathrm{C}-\mathrm{F},{ }^{1} J_{\mathrm{C}-\mathrm{F}}=270.2 \mathrm{~Hz}\right)$, 120.7, 114.5, 113.1, 61.1, 55.5, 30.5, 14.1; HRMS (ESI) m/z: $[\mathrm{M}+\mathrm{H}]^{+}$Calcd for $\mathrm{C}_{21} \mathrm{H}_{20} \mathrm{~N}_{2} \mathrm{O}_{3} \mathrm{~F}_{3}$ 405.1421; Found 405.1424.<smiles>CCOC(=O)Cc1cn(-c2ccc(OC)cc2)nc1-c1ccc([N+](=O)[O-])cc1</smiles>

[1-(4-Methoxyphenyl)-3-(4-nitrophenyl)-1 $\boldsymbol{H}$-pyrazol-4-yl]acetic acid ethyl ester (5jO) $37.8 \mathrm{mg}$, 99\% yield; Reaction time: $2 \mathrm{~h}$; Purification by preparative TLC $(n$-hexane: EtOAc $=2: 1$ ); An orange oil; IR (neat): $1736 \mathrm{~cm}^{-1} ;{ }^{1} \mathrm{H}$ NMR (300 MHz, $\left.\mathrm{CDCl}_{3}\right) \delta: 8.28(\mathrm{~d}, J=9.0 \mathrm{~Hz}, 2 \mathrm{H}), 7.96(\mathrm{~s}, 1 \mathrm{H}), 7.90$ (d, $J=9.0 \mathrm{~Hz}, 2 \mathrm{H}), 7.63$ (d, $J=9.0 \mathrm{~Hz}, 2 \mathrm{H}), 6.97$ (d, $J=9.0 \mathrm{~Hz}, 2 \mathrm{H}), 4.18$ (q, $J=7.2 \mathrm{~Hz}, 2 \mathrm{H}), 3.85$ (s, 3H), $3.72(\mathrm{~s}, 2 \mathrm{H}), 1.26$ (t, $J=7.2 \mathrm{~Hz}, 3 \mathrm{H}) ;{ }^{13} \mathrm{C} \mathrm{NMR}\left(75 \mathrm{MHz}, \mathrm{CDCl}_{3}\right) \delta: 171.0,158.6,149.0$, $147.1,139.8,133.4,128.7,128.4,123.9,120.8,114.6,113.5,61.3,55.6,30.6,14.2$; HRMS (ESI) $\mathrm{m} / \mathrm{z}:[\mathrm{M}+\mathrm{H}]^{+}$Calcd for $\mathrm{C}_{20} \mathrm{H}_{20} \mathrm{~N}_{3} \mathrm{O}_{5} 382.1397$; Found 382.1401 .<smiles>CCOCc1cn(-c2ccc(OC)cc2)nc1-c1cccc(Cl)c1</smiles>

[3-(3-Chlorophenyl)-1-(4-methoxyphenyl)-1H-pyrazol-4-yl]acetic acid ethyl ester (5jP) $27.0 \mathrm{mg}$, $72 \%$ yield; Reaction time: 2 h; Purification by preparative TLC ( $n$-hexane: EtOAc $=2: 1$ ); A yellow 
oil; IR (neat): $1736 \mathrm{~cm}^{-1} ;{ }^{1} \mathrm{H}$ NMR $\left(300 \mathrm{MHz}, \mathrm{CDCl}_{3}\right) \delta: 7.94(\mathrm{~s}, 1 \mathrm{H}), 7.72(\mathrm{~s}, 1 \mathrm{H}), 7.63(\mathrm{~d}, J=9.3$ $\mathrm{Hz}, 2 \mathrm{H}), 7.59-7.56(\mathrm{~m}, 1 \mathrm{H}), 7.40-7.31(\mathrm{~m}, 2 \mathrm{H}), 6.97$ (d, $J=9.0 \mathrm{~Hz}, 2 \mathrm{H}), 4.18$ (q, $J=7.2 \mathrm{~Hz}, 2 \mathrm{H})$, $3.84(\mathrm{~s}, 3 \mathrm{H}), 3.68$ (s, $2 \mathrm{H}), 1.26$ (t, $J=7.2 \mathrm{~Hz}, 3 \mathrm{H}) ;{ }^{13} \mathrm{C} \mathrm{NMR}\left(75 \mathrm{MHz}, \mathrm{CDCl}_{3}\right) \delta: 171.0,158.0$, 149.9, 134.7, 134.2, 133.4, 129.5, 127.9, 127.8, 127.7, 125.9, 120.5, 114.3, 112.7, 61.2, 55.6, 30.6, 14.4; HRMS (ESI) m/z: [M+ Na] $]^{+}$Calcd for $\mathrm{C}_{20} \mathrm{H}_{19} \mathrm{~N}_{2} \mathrm{O}_{3}{ }^{35} \mathrm{ClNa}$ 393.0976; Found 393.0981.<smiles>CCOCc1cn(-c2ccc(OC)cc2)nc1-c1ccccc1O</smiles>

[3-(2-Hydroxyphenyl)-1-(4-methoxyphenyl)-1H-pyrazol-4-yl]acetic acid ethyl ester (5jQ) 18.5 mg, 53\% yield; Reaction time: $2 \mathrm{~h}$; Purification by preparative TLC ( $n$-hexane: EtOAc $=2: 1)$; An orange oil; IR (neat): $3134,1733 \mathrm{~cm}^{-1} ;{ }^{1} \mathrm{H}$ NMR $\left(300 \mathrm{MHz}, \mathrm{CDCl}_{3}\right) \delta: 10.60$ (br s, $1 \mathrm{H}$ ), 7.98 (s, $1 \mathrm{H}$ ), $7.55(\mathrm{~d}, J=9.0 \mathrm{~Hz}, 2 \mathrm{H}), 7.51$ (d, $J=7.8 \mathrm{~Hz}, 1 \mathrm{H}), 7.24$ (t, $J=7.5 \mathrm{~Hz}, 1 \mathrm{H}), 7.05$ (d, $J=8.1 \mathrm{~Hz}, 1 \mathrm{H})$, $6.96(\mathrm{~d}, J=9.0 \mathrm{~Hz}, 2 \mathrm{H}), 6.91(\mathrm{~d}, J=7.2 \mathrm{~Hz}, 1 \mathrm{H}), 4.19$ (q, $J=7.2 \mathrm{~Hz}, 2 \mathrm{H}), 3.84(\mathrm{~s}, 3 \mathrm{H}), 3.80$ (s, 2H), $1.26(\mathrm{t}, J=7.2 \mathrm{~Hz}, 3 \mathrm{H}) ;{ }^{13} \mathrm{C}$ NMR $\left(75 \mathrm{MHz}, \mathrm{CDCl}_{3}\right) \delta: 170.7,158.1,155.5,148.9,132.6,129.2$, 128.4 127.0, 120.2, 119.1, 117.0, 116.9, 114.5, 112.9, 61.3, 55.7, 31.4, 14.4; HRMS (ESI) m/z: [M + $\mathrm{H}]^{+}$Calcd for $\mathrm{C}_{20} \mathrm{H}_{21} \mathrm{~N}_{2} \mathrm{O}_{4}$ 353.1496; Found 353.1498.

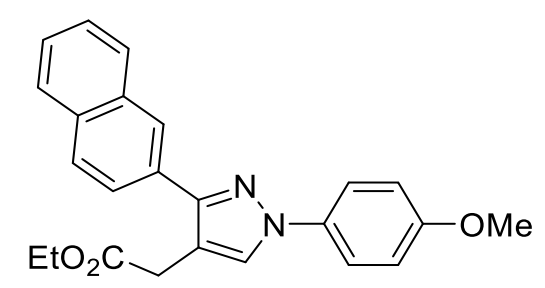

[1-(4-Methoxyphenyl)-3-(2-naphthalenyl)-1H-pyrazol-4-]acetic acid ethyl ester (5jR) $29.7 \mathrm{mg}$, $76 \%$ yield; Reaction time: $2 \mathrm{~h}$; Purification by preparative TLC ( $n$-hexane: EtOAc $=2: 1$ ); A yellow oil; IR (neat): $1733 \mathrm{~cm}^{-1}$; ${ }^{1} \mathrm{H}$ NMR $\left(300 \mathrm{MHz}, \mathrm{CDCl}_{3}\right) \delta: 8.11(\mathrm{~s}, 1 \mathrm{H}), 7.97(\mathrm{~s}, 1 \mathrm{H}), 7.91-7.83(\mathrm{~m}$, 4H), $7.66(\mathrm{~d}, J=8.7 \mathrm{~Hz}, 2 \mathrm{H}), 7.50-7.44(\mathrm{~m}, 2 \mathrm{H}), 6.96(\mathrm{~d}, J=8.7 \mathrm{~Hz}, 2 \mathrm{H}), 4.17$ (q, $J=7.2 \mathrm{~Hz}, 2 \mathrm{H})$, $3.84(\mathrm{~s}, 3 \mathrm{H}), 3.77(\mathrm{~s}, 2 \mathrm{H}), 1.25$ (t, $J=7.2 \mathrm{~Hz}, 3 \mathrm{H}) ;{ }^{13} \mathrm{C} \mathrm{NMR}\left(75 \mathrm{MHz}, \mathrm{CDCl}_{3}\right) \delta: 171.2,157.9$, $151.2,133.6,133.2,132.7,130.4,128.1,128.0,127.9,127.5,126.7,126.0,125.9,125.8,120.5$, 114.3, 112.9, 61.1, 55.6, 30.9, 14.4; HRMS (ESI) m/z: $[\mathrm{M}+\mathrm{H}]^{+}$Calcd for $\mathrm{C}_{24} \mathrm{H}_{23} \mathrm{~N}_{2} \mathrm{O}_{3}$ 409.1523; Found 409.1522 . 


\subsection{Synthesis of Lonazolac.}<smiles>CCOC(=O)Cc1cn(-c2ccccc2)nc1-c1ccc(Cl)cc1</smiles>

To a solution of conjugated hydrazone $\mathbf{1 j}(52.5 \mathrm{mg}, 0.20 \mathrm{mmol})$ and hydrazone $4 \mathrm{~S}(55.4 \mathrm{mg}, 0.24$ $\mathrm{mmol})$ in $\mathrm{CH}_{2} \mathrm{Cl}_{2}(2.0 \mathrm{~mL})$ was added $\mathrm{MsOH}(26 \mu \mathrm{L}, 0.40 \mathrm{mmol})$ at room temperature. After being stirred at the same temperature for $6 \mathrm{~h}$, the reaction mixture was diluted with $10 \% \mathrm{HCl}(5 \mathrm{~mL})$ and extracted with $\mathrm{CHCl}_{3}(30 \mathrm{~mL} \times 3)$. The organic phase was dried over $\mathrm{MgSO}_{4}$ and concentrated under reduced pressure. The crude product was purified by preparative TLC $\left(\mathrm{CHCl}_{3}\right)$ to afford pyrazole 5jS (25.2 mg, 37\% yield) as a yellow oil; IR (neat): $1736 \mathrm{~cm}^{-1} ;{ }^{1} \mathrm{H}$ NMR $\left(300 \mathrm{MHz}, \mathrm{CDCl}_{3}\right) \delta: 8.04$ (s, $1 \mathrm{H}), 7.74(\mathrm{~d}, J=8.7 \mathrm{~Hz}, 2 \mathrm{H}), 7.65(\mathrm{~d}, J=8.7 \mathrm{~Hz}, 2 \mathrm{H}), 7.48-7.40(\mathrm{~m}, 4 \mathrm{H}), 7.31-7.26(\mathrm{~m}, 1 \mathrm{H})$, 4.18 (q, $J=7.2 \mathrm{~Hz}, 2 \mathrm{H}), 3.67$ (s, $2 \mathrm{H}), 1.25(\mathrm{t}, J=7.2 \mathrm{~Hz}, 3 \mathrm{H}) ;{ }^{13} \mathrm{C} \mathrm{NMR}\left(75 \mathrm{MHz}, \mathrm{CDCl}_{3}\right) \delta: 171.2$, $150.8,139.8,133.9,131.5,129.4,129.3,128.7,128.0,126.5,118.9,113.2,61.1,30.5,14.1$; HRMS (ESI) $\mathrm{m} / \mathrm{z}:[\mathrm{M}+\mathrm{H}]^{+}$Calcd for $\mathrm{C}_{19} \mathrm{H}_{18} \mathrm{~N}_{2} \mathrm{O}_{2}{ }^{35} \mathrm{Cl} 341.1051$; Found 341.1054 .<smiles>CCOCc1cn(-c2ccccc2)nc1-c1ccc(Cl)cc1</smiles>

$5 \mathrm{jS}$

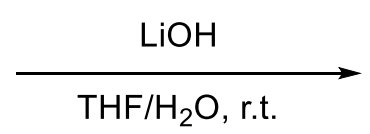

$\mathrm{HO}_{2} \mathrm{C}$

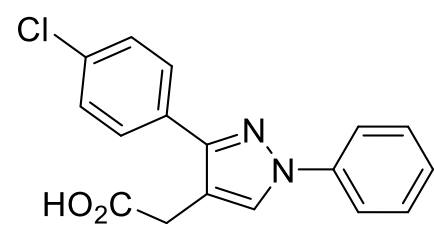

Lonazolac

To a solution of pyrazole $\mathbf{5 j S}(22.7 \mathrm{mg}, 0.067 \mathrm{mmol})$ in THF $(2.0 \mathrm{~mL})$ was added LiOH $(6.7 \mathrm{mg}$, $0.28 \mathrm{mmol})$ in $\mathrm{H}_{2} \mathrm{O}(0.50 \mathrm{~mL})$ at room temperature. After being stirred at the same temperature for 3 $\mathrm{h}$, the reaction mixture was acidified with $10 \% \mathrm{HCl}$ until $\mathrm{pH} 1-2$ and extracted with $\mathrm{CHCl}_{3}(30 \mathrm{~mL}$ $\times 3$ ). The organic phase was dried over $\mathrm{MgSO}_{4}$ and concentrated under reduced pressure. The crude product was purified by preparative TLC $\left(\mathrm{CHCl}_{3}: \mathrm{MeOH}=10: 1\right)$ to afford the Lonazolac ${ }^{2}(18.6 \mathrm{mg}$, $89 \%$ yield) as a white solid; Mp: $148-149{ }^{\circ} \mathrm{C}(\mathrm{MeOH})$; IR (KBr): 3430, $1723 \mathrm{~cm}^{-1} ;{ }^{1} \mathrm{H}$ NMR (300 MHz, DMSO- $\left.d_{6}\right) \delta: 8.50(\mathrm{~s}, 1 \mathrm{H}), 7.87(\mathrm{~d}, J=8.1 \mathrm{~Hz}, 2 \mathrm{H}), 7.75(\mathrm{~d}, J=8.4 \mathrm{~Hz}, 2 \mathrm{H}), 7.55-7.49(\mathrm{~m}$, 4H), 7.32 (t, $J=7.2 \mathrm{~Hz}, 1 \mathrm{H}), 3.69$ (s, 2H); ${ }^{13} \mathrm{C}$ NMR (75 MHz, DMSO-d 6 ) $\delta: 172.3,149.7,139.3$, 132.6, 131.9, 129.6, 129.2, 129.0, 128.6, 126.3, 118.1, 114.3, 30.1; HRMS (ESI) m/z: $[\mathrm{M}+\mathrm{H}]^{+}$ Calcd for $\mathrm{C}_{17} \mathrm{H}_{14} \mathrm{~N}_{2} \mathrm{O}_{2}{ }^{35} \mathrm{Cl}$ 313.0738; Found 313.0740. 


\subsection{Control experiments.}<smiles>CCOC(=O)/C=C/C=N/Nc1ccccc1</smiles>

$1 \mathrm{a}$<smiles>CCOC(=O)CC=Cc1cn(-c2ccccc2)nc1CC(=O)OCC</smiles>

2a<smiles>[2H]C(=O)C(CC)c1cn(-c2ccccc2)nc1/C=C/COCC</smiles>

2a': $33 \%, 72 \%$ D

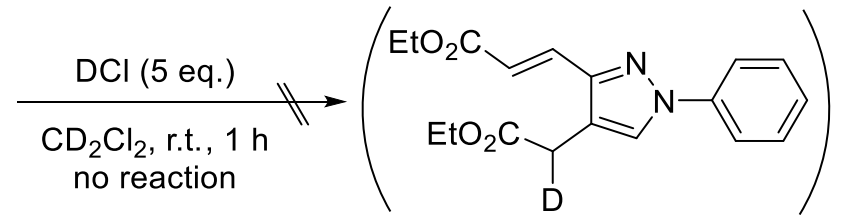

2a': not detected

(eq. 1)

The reaction of $1 \mathrm{a}$ with $\mathrm{DCl}\left(1.0 \mathrm{M}\right.$ in $\mathrm{Et}_{2} \mathrm{O}, 5$ eq.) in $\mathrm{CD}_{2} \mathrm{Cl}_{2}$ at room temperature afforded pyrazole 2a' in $33 \%$ yield ( $72 \% \mathrm{D}$, estimated by ${ }^{1} \mathrm{H}$ NMR) (eq. 1). In contrast, the deuterium exchange reaction of $2 \mathrm{a}$ with $\mathrm{DCl}$ in $\mathrm{CD}_{2} \mathrm{Cl}_{2}$ did not proceeded (eq. 2). These results indicated that the corresponding 1,2-diaza-1,3-diene I was generated by $\beta$-protonation of $\alpha, \beta$-unsaturated hydrazone 1a. 


\subsection{MS experiment.}

The ESI (+)-MS spectrum of reaction mixture showed a peak of $\mathrm{m} / \mathrm{z} 497.23822$ which indicated the intermediate $\mathbf{I I}+\mathrm{H}^{+}, \mathbf{I I I}+\mathrm{H}^{+}$or $\mathbf{I V}+\mathrm{H}^{+}$.

Reaction conditions: hydrazone 1a, $\mathrm{MsOH}$ (1.0 eq.), $\mathrm{CH}_{2} \mathrm{Cl}_{2}, \mathrm{rt}, 10 \mathrm{~min}$.

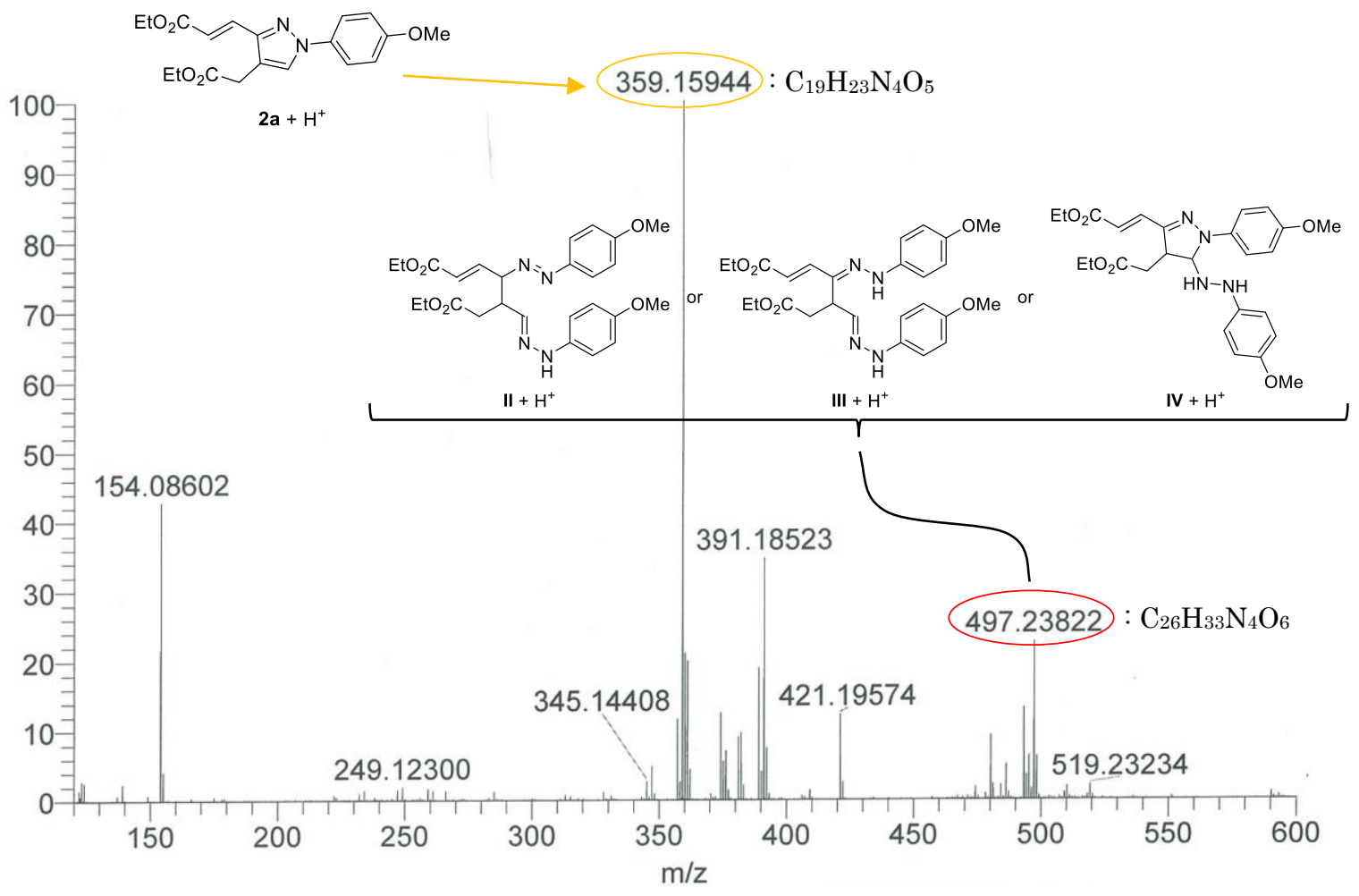

\section{References}

(1) a) Y. Ito, M. Ueda, N. Takeda, O. Miyata. Chem. Eur. J. 2016, 22, 2616. b) W. Wu, X. Yuan, J. Hu, X. Wu, Y. Wei, Z. Liu, J. Lu, and J. Ye. Org. Lett. 2013, 15, 4524. c) J.-R. Hu, W.-J. Zhang, D.-G. Zheng. Tetrahedron 2013, 69, 9865. d) R. N. Rutte, T. B. Parsons, B. G. Davis. Chem. Commun. 2014, 50, 12297. e) X. Deng, N. S. Mani. J. Org. Chem. 2008, 73, 2412. f) P. Li, C. Wu, J. Zhao, D. C. Rogness, F. Shi. J. Org. Chem. 2012, 77, 3149. g) J. L. Delgado, F. Oswald, F. Cardinali, F. Langa, N. Martín. J. Org. Chem. 2008, 73, 3184.

(2) M. V. Vovk, V. O. Chornous, I. F. Tsimbal, M. K. Bratenko. Ukr. Khim. Zh., 2002, 68, 59. 


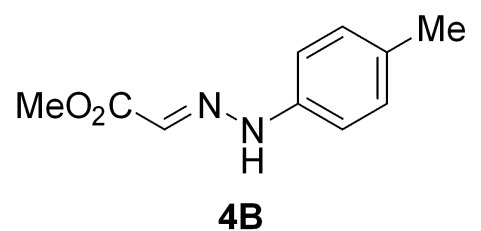

(300 MHz in DMSO- $d_{6}$ )

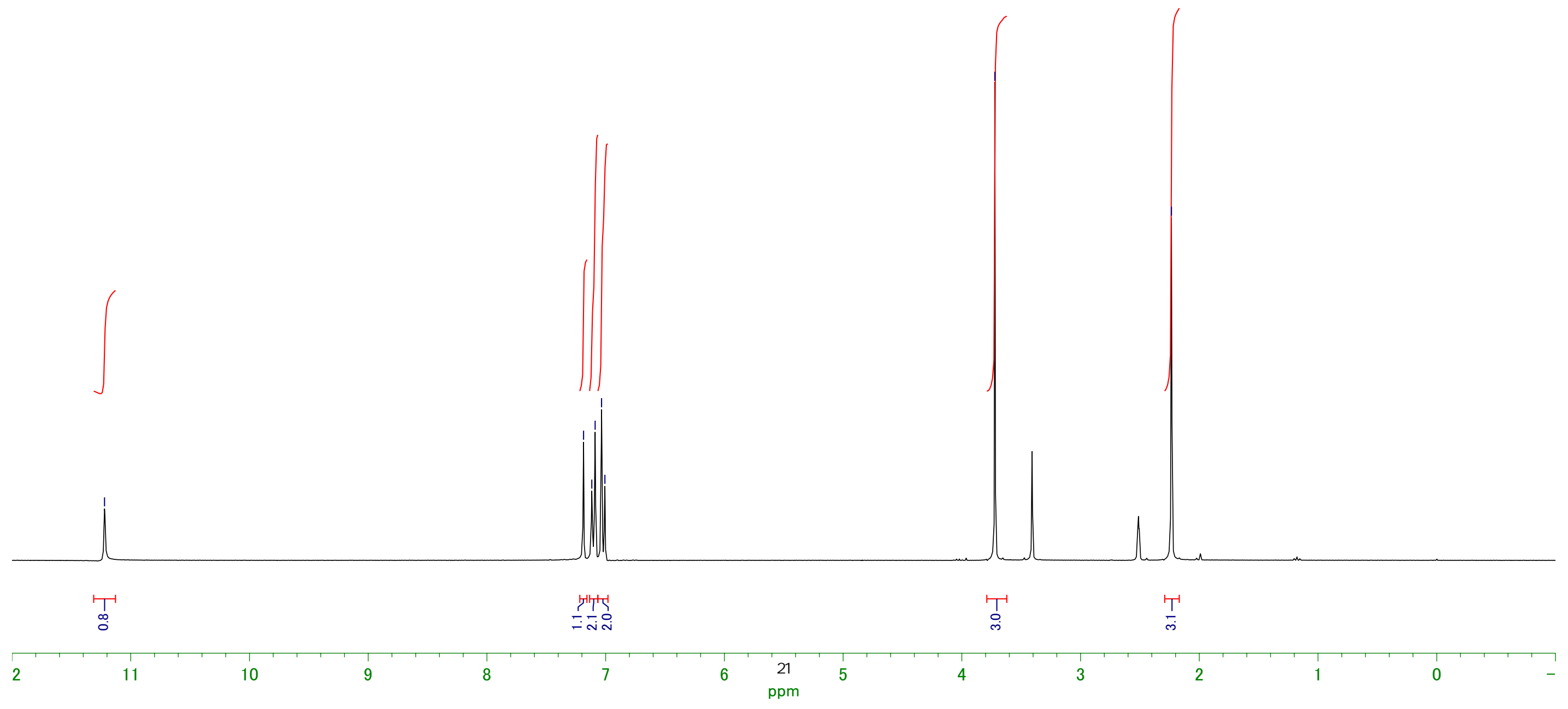




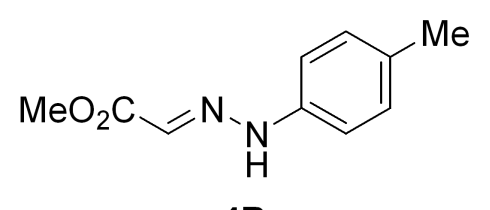

4B

$\left(75 \mathrm{MHz}\right.$ in DMSO- $\left.d_{6}\right)$ 
$\mathrm{MeO}_{2} \mathrm{C} \backslash \mathrm{C}_{4 \mathrm{C}}^{t-\mathrm{Bu}}$

(300 MHz in $\mathrm{CHCl}_{3}$ )

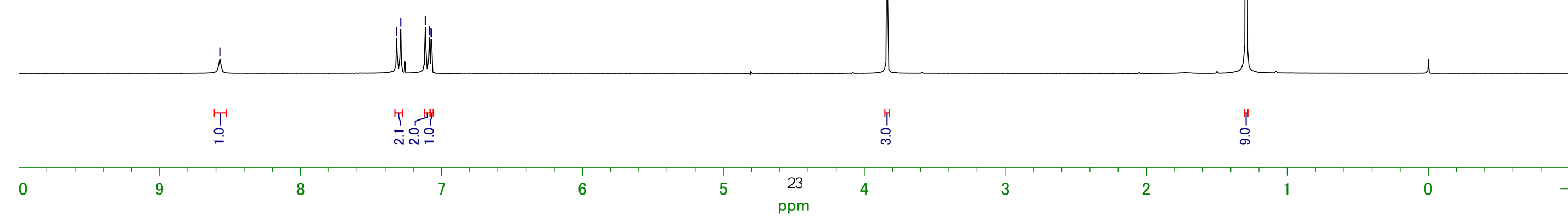




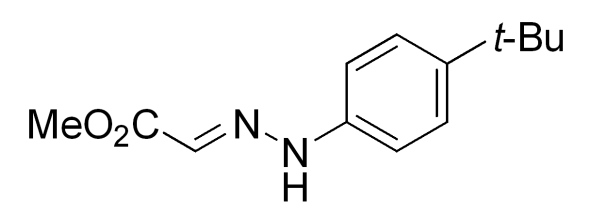

4C

$\left(75 \mathrm{MHz}\right.$ in $\left.\mathrm{CHCl}_{3}\right)$ 
$\mathrm{MeO}_{2} \mathrm{C}=\mathrm{N}$

4D

(300 MHz in DMSO-d $\left._{6}\right)$

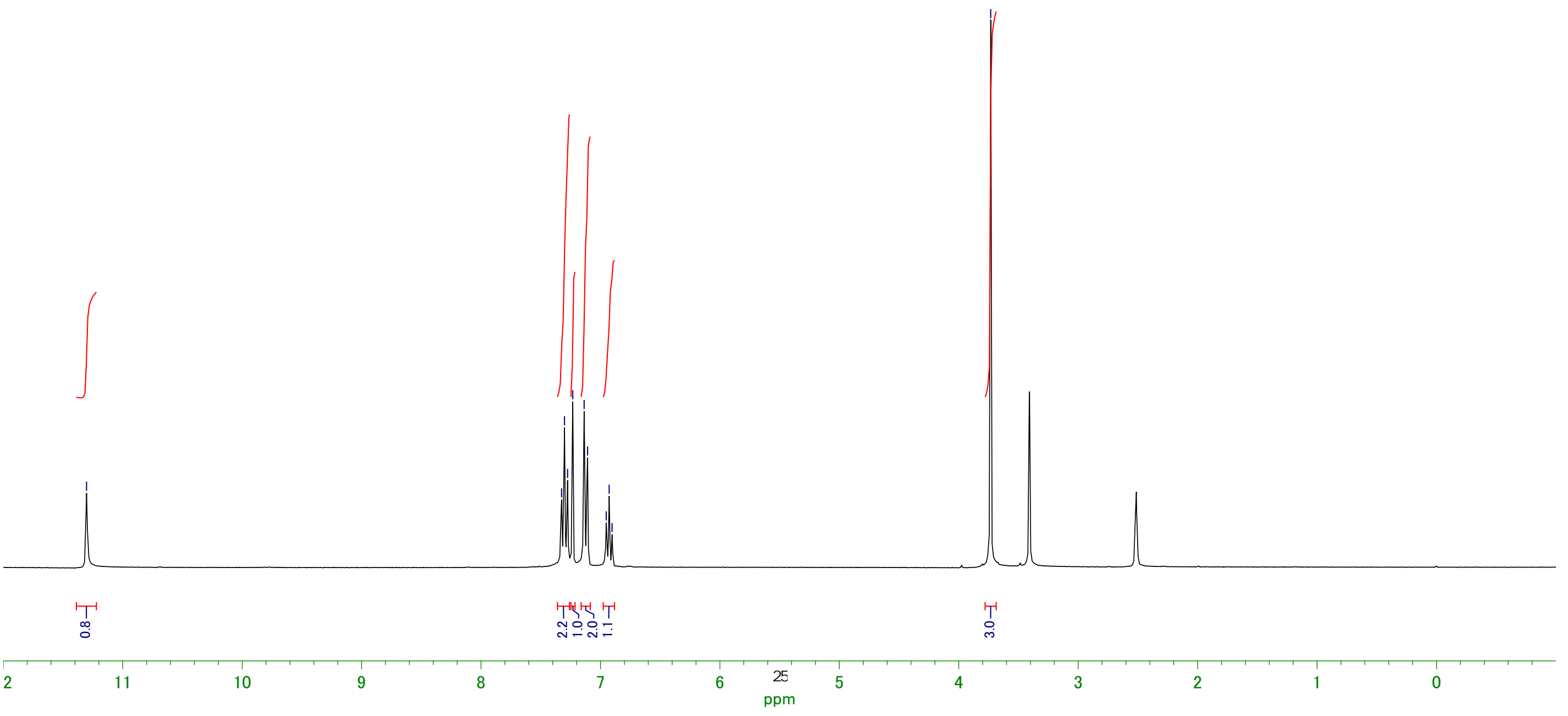




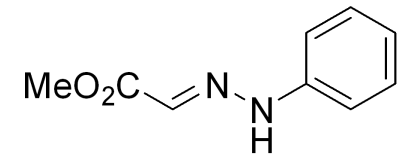

4D

(75 $\mathrm{MHz}$ in $\mathrm{CHCl}_{3}$ )

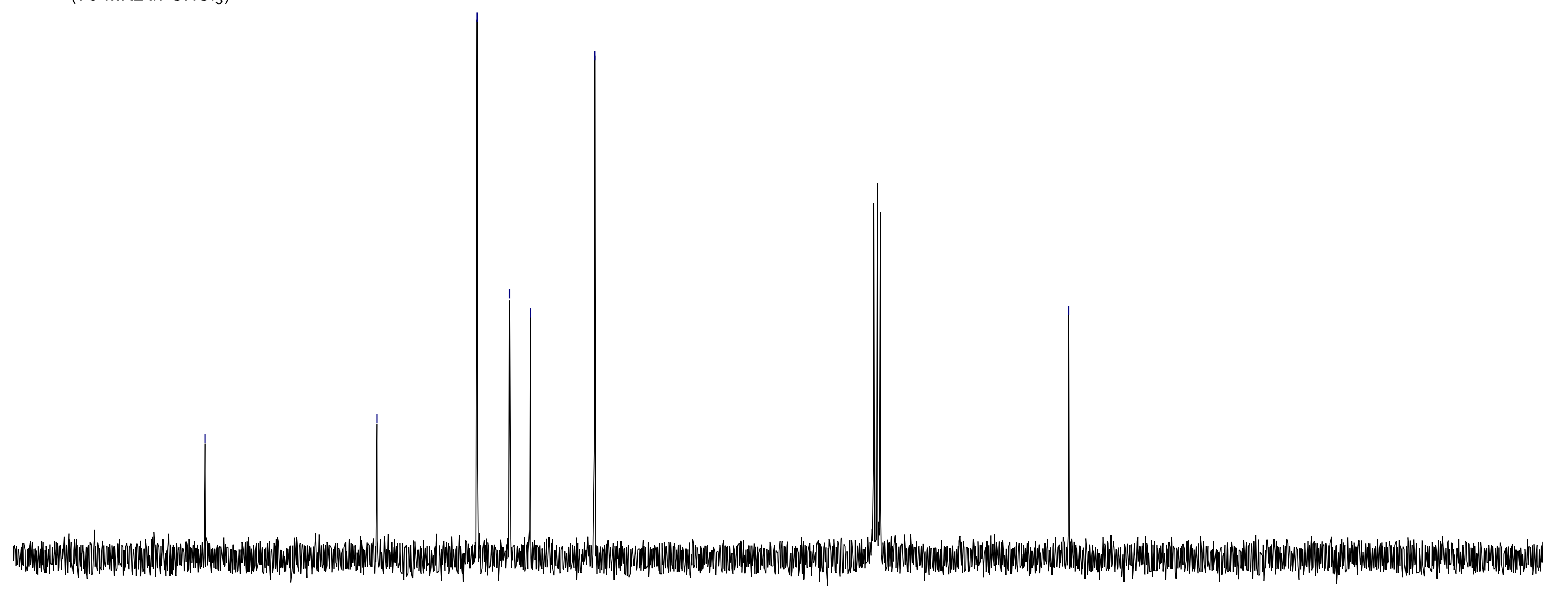


$\mathrm{MeO}_{2} \mathrm{C}=\mathrm{N}_{\mathrm{H}}$

(300 $\mathrm{MHz}$ in $\left.\mathrm{CHCl}_{3}\right)$

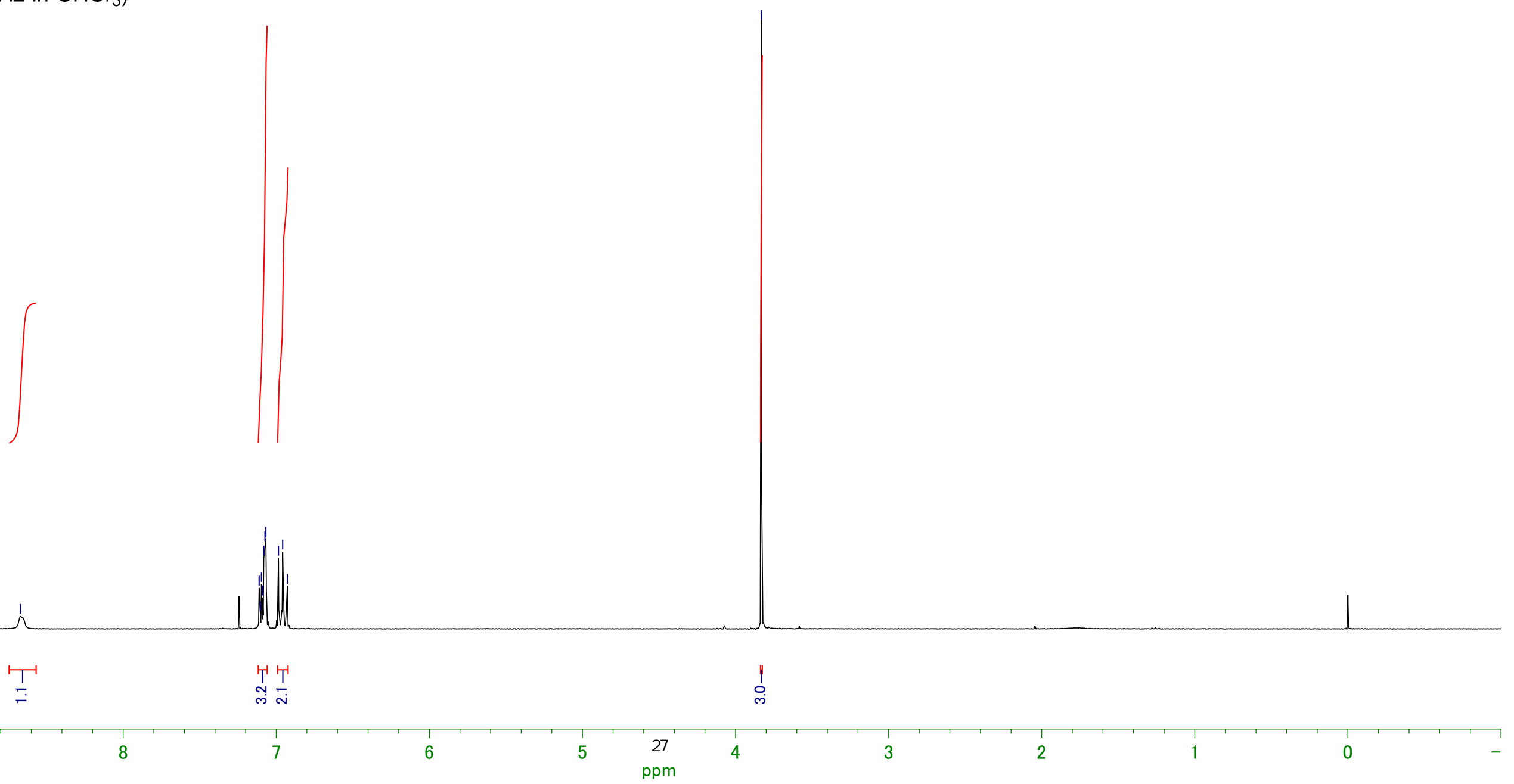




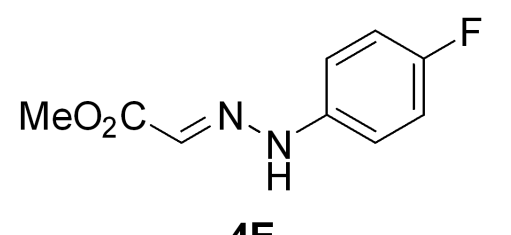

$\left(150 \mathrm{MHz}\right.$ in $\left.\mathrm{CHCl}_{3}\right)$ 

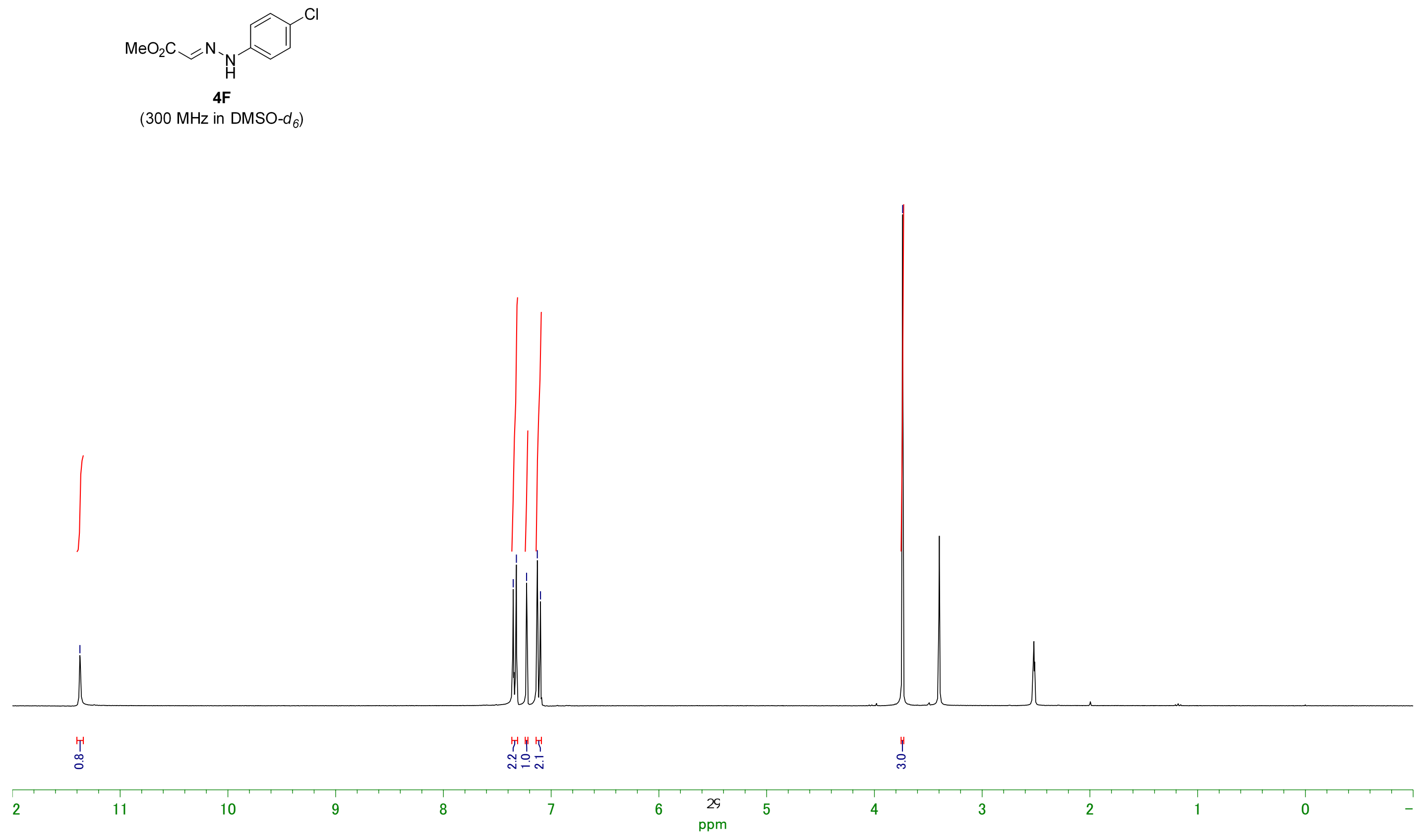


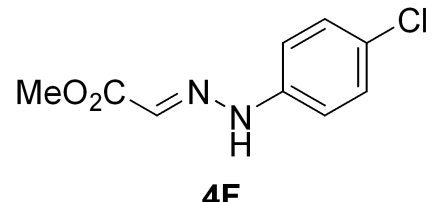

$(75 \mathrm{MHz}$ in DMSO-d $)$

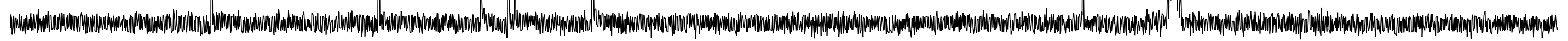



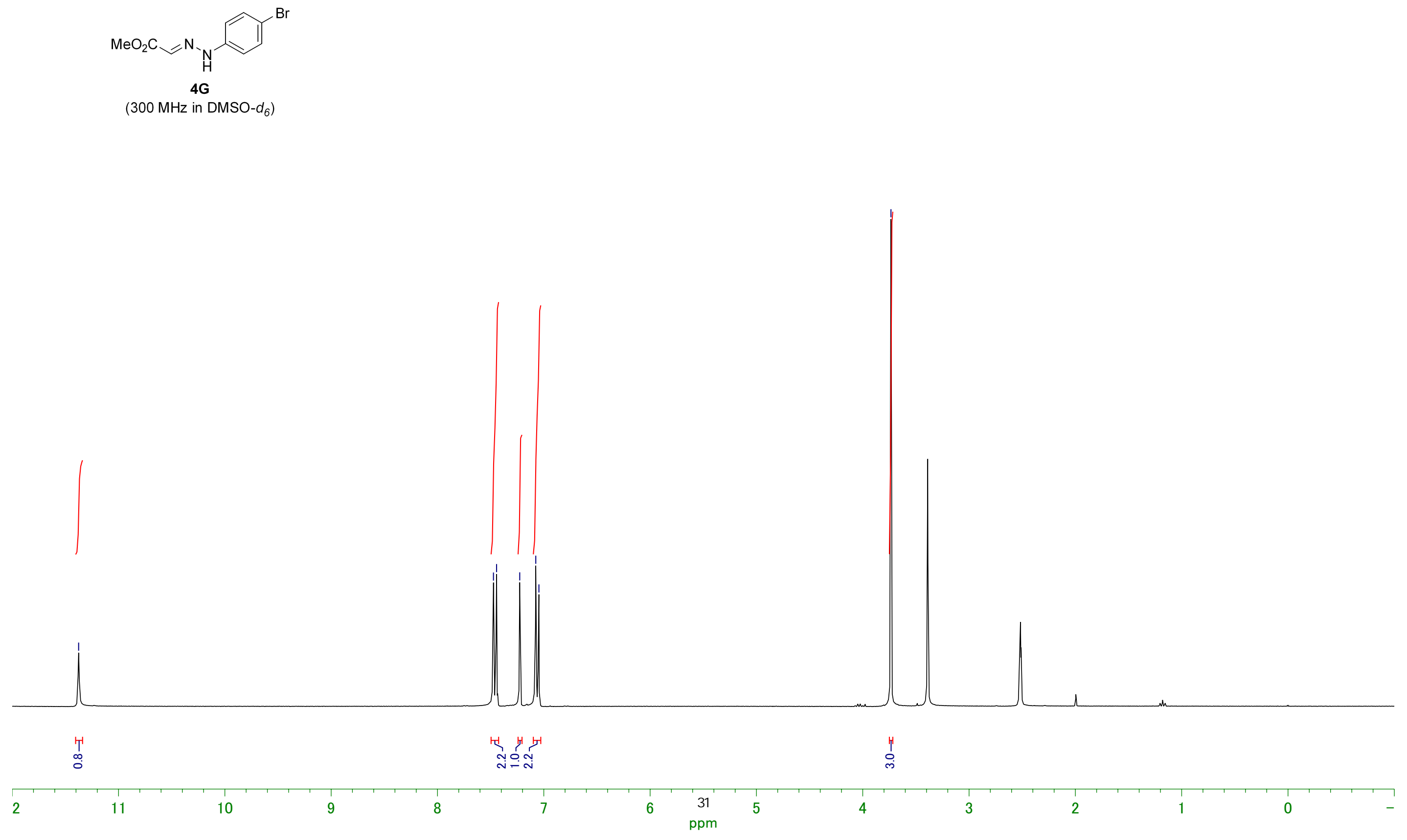


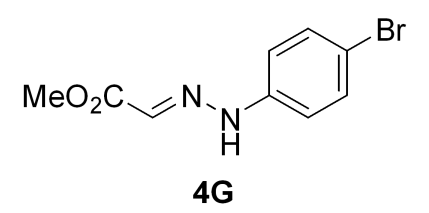

(75 MHz in DMSO- $d_{6}$ )

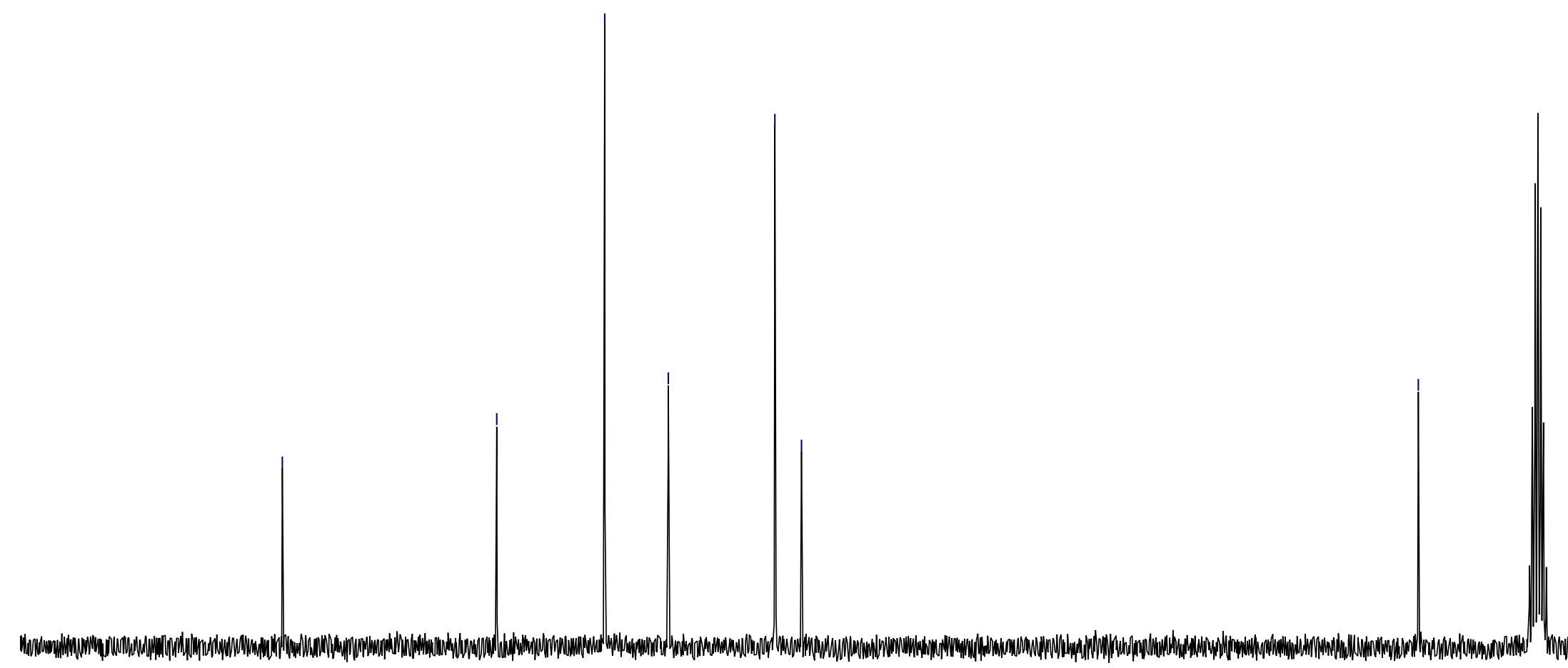

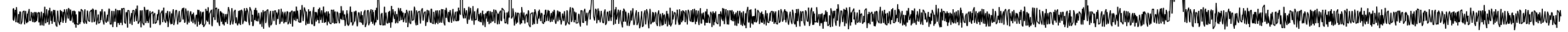




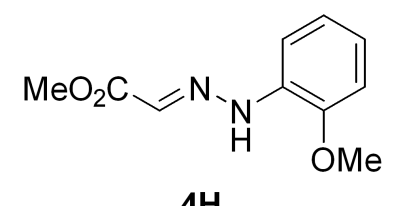

(300 $\mathrm{MHz}$ in $\mathrm{CHCl}_{3}$ )
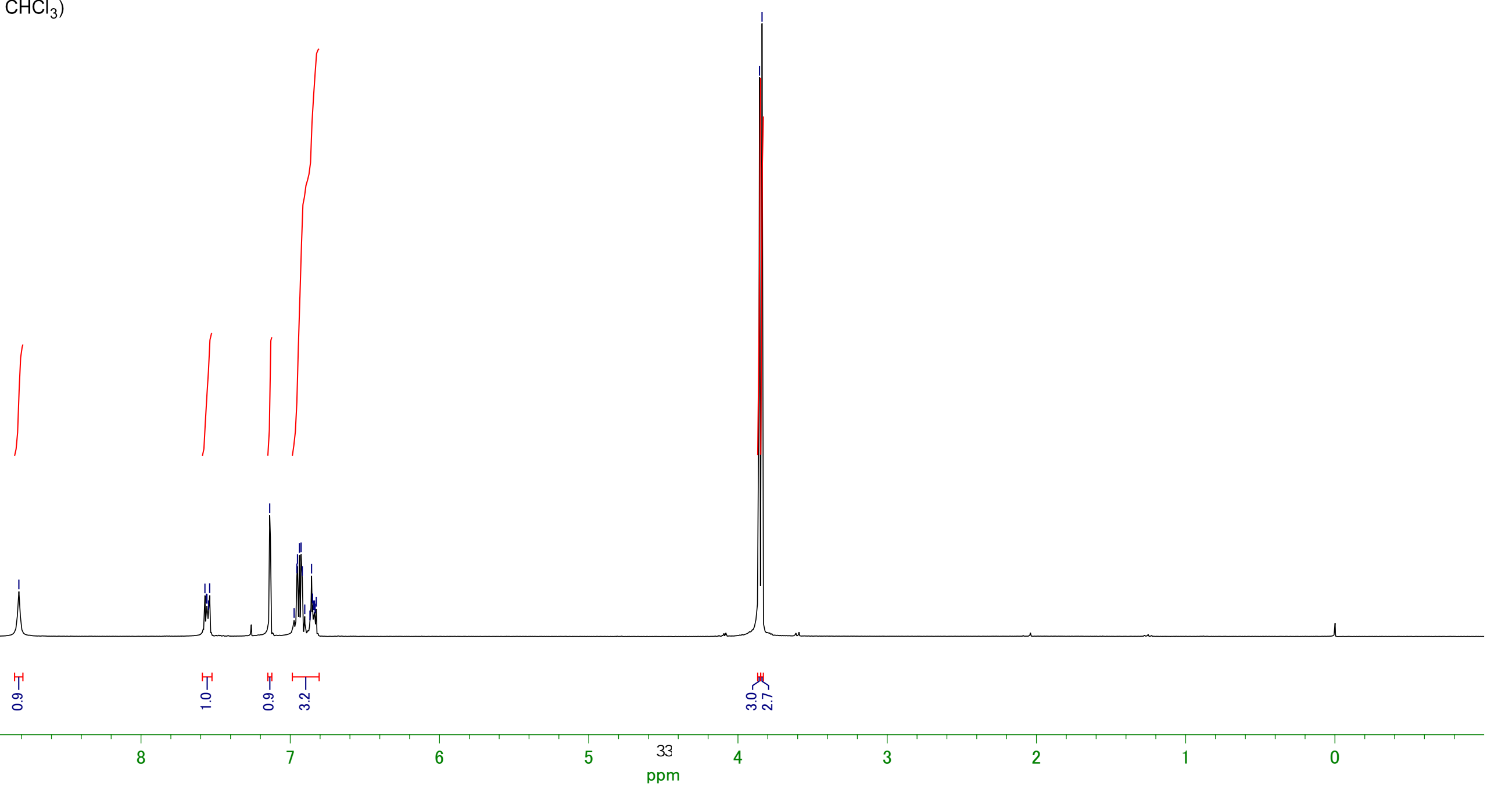


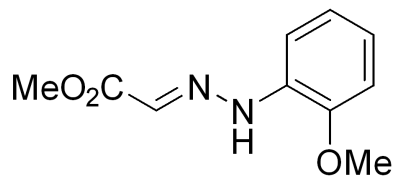
4H

(75 $\mathrm{MHz}$ in $\mathrm{CHCl}_{3}$ )

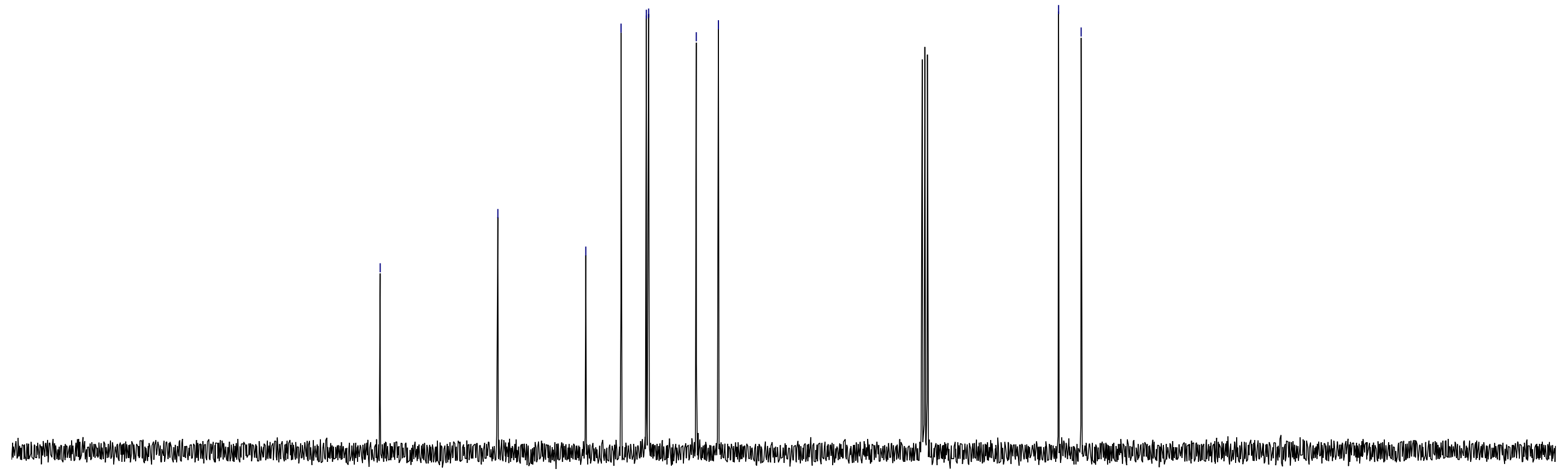


$\mathrm{Me}$

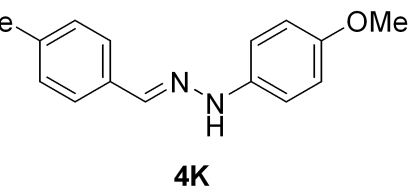

(300 MHz in DMSO- $d_{6}$ )

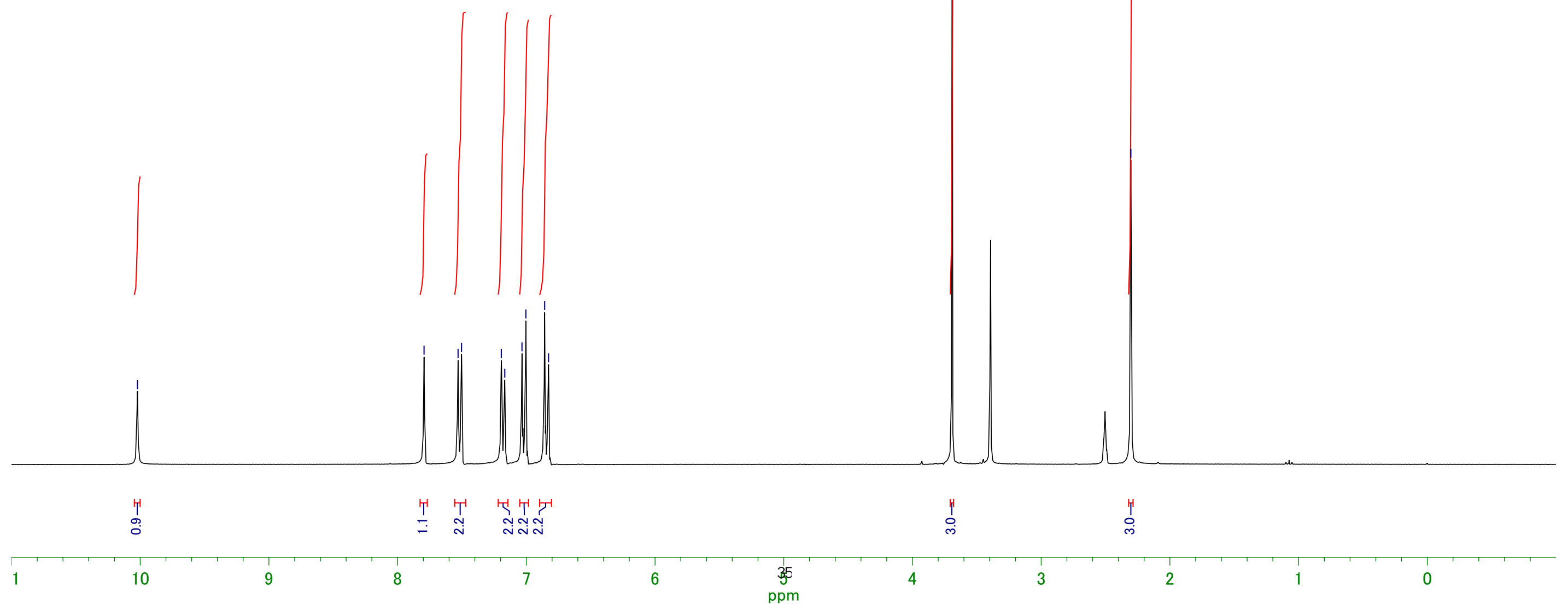




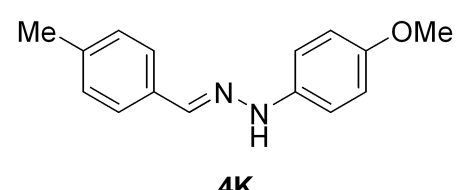

(75 MHz in DMSO- $d_{6}$ )

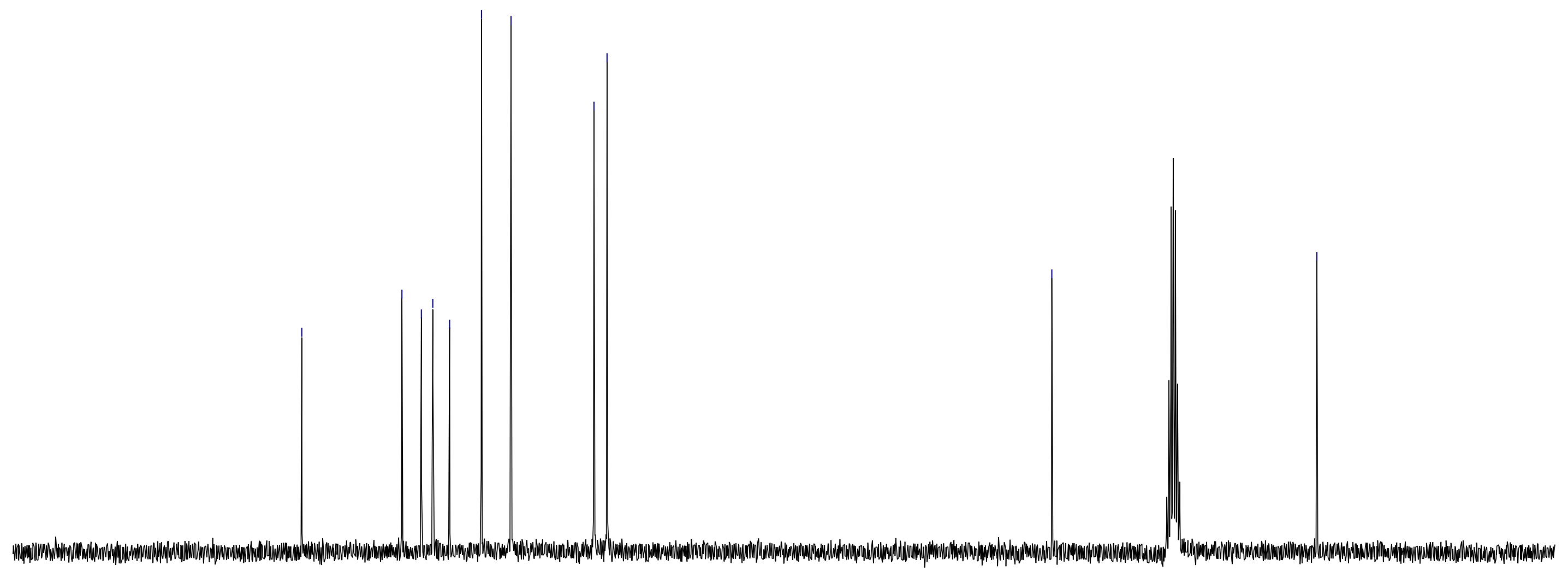


${ }^{\mathrm{F}_{3} \mathrm{C}}$ $4 \mathrm{~N}$

(300 MHz in DMSO- $d_{6}$ )

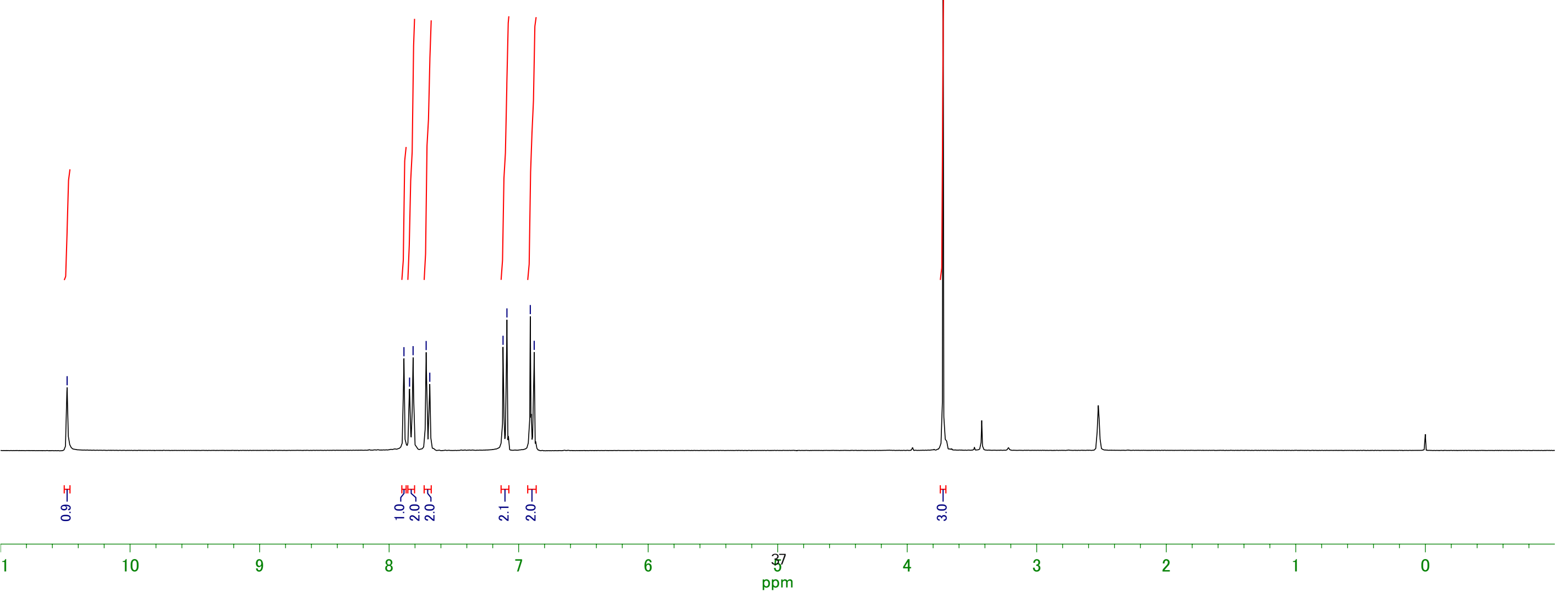




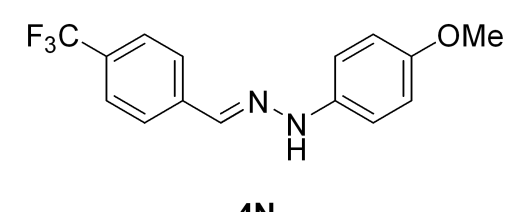

(150 MHz in DMSO-d $d_{6}$ )

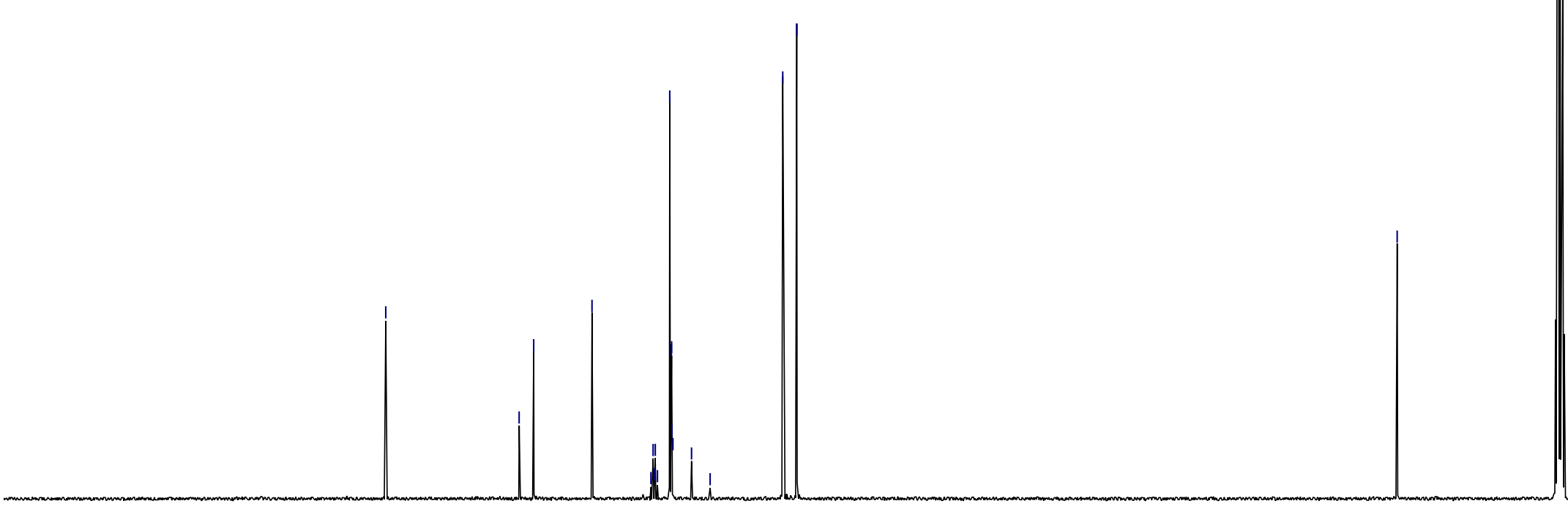




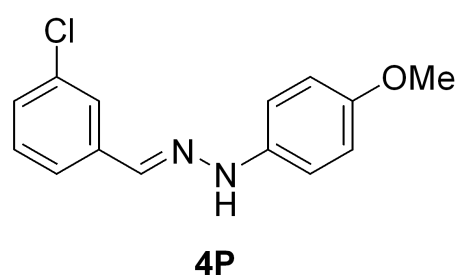

(300 $\mathrm{MHz}$ in DMSO- $d_{6}$ )

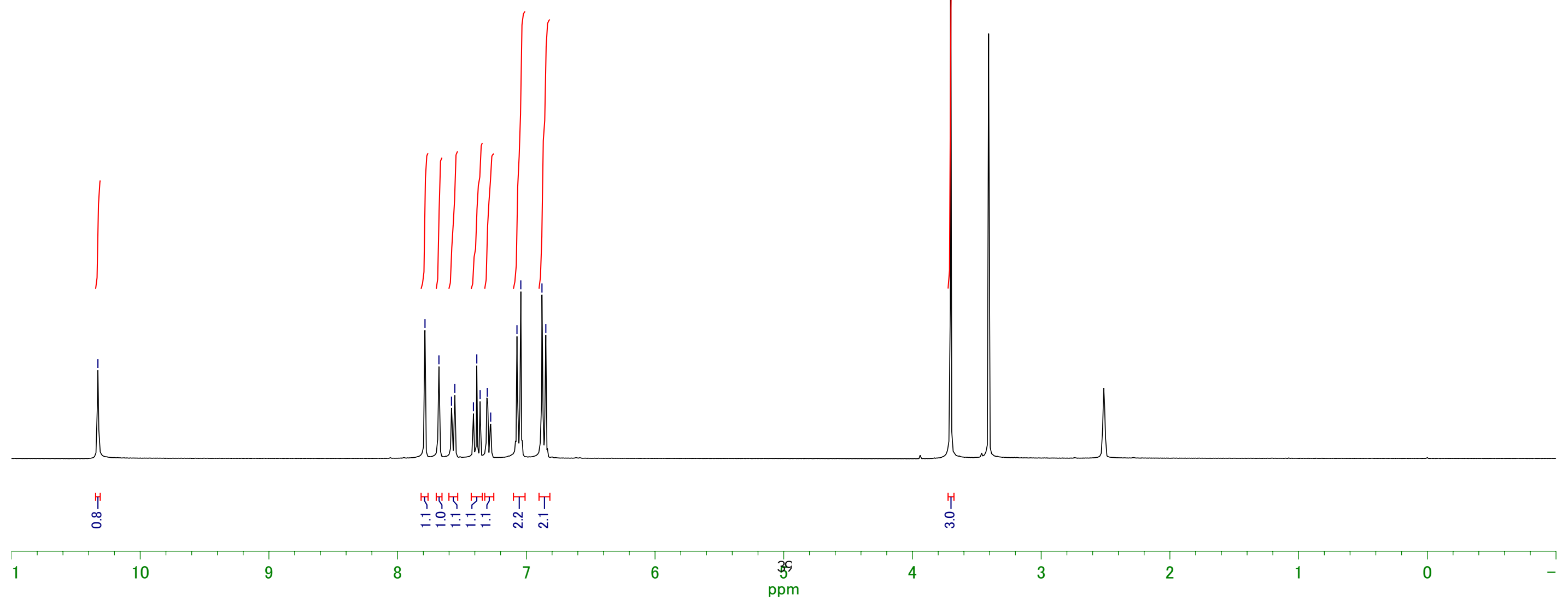




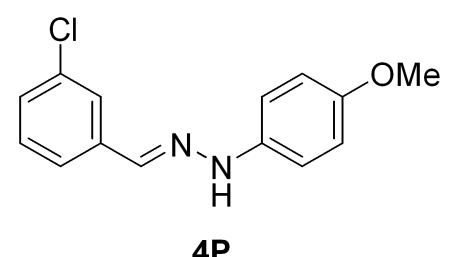

$\left(75 \mathrm{MHz}\right.$ in $\left.\mathrm{DMSO}-d_{6}\right)$

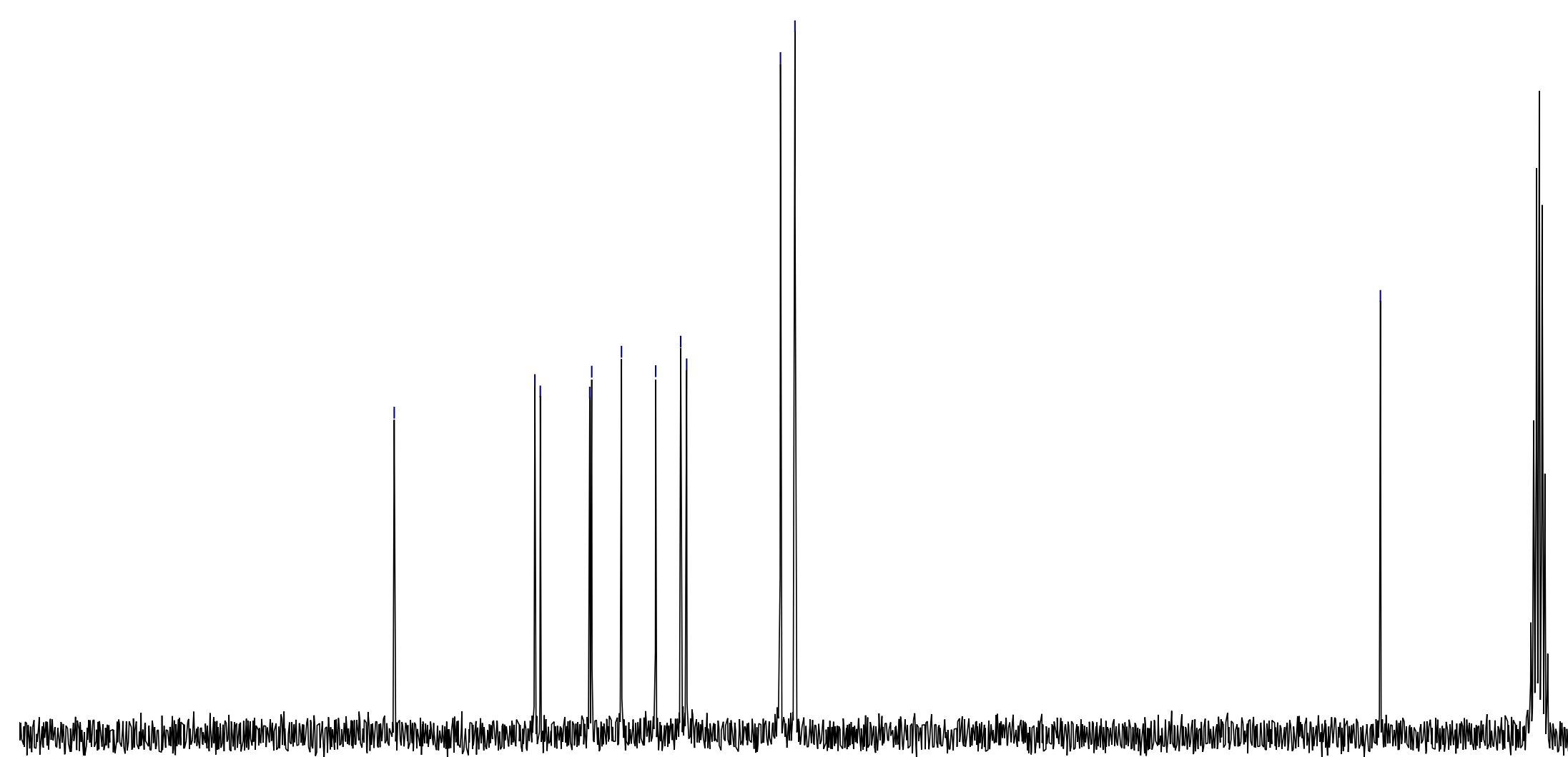



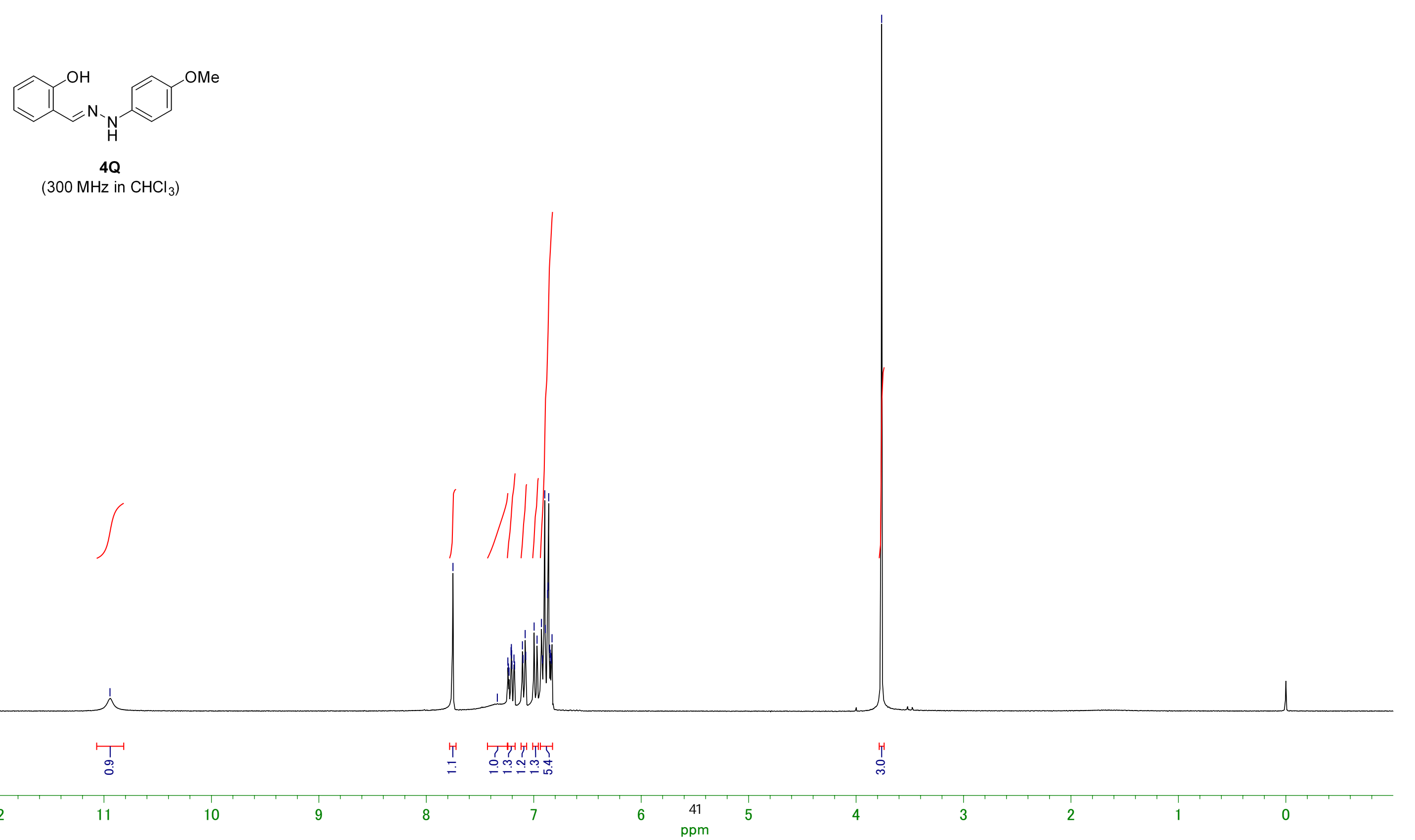


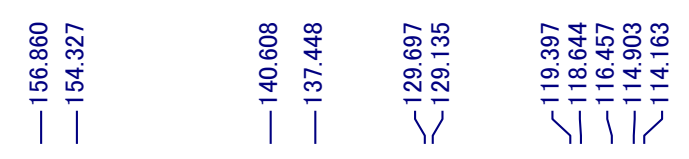

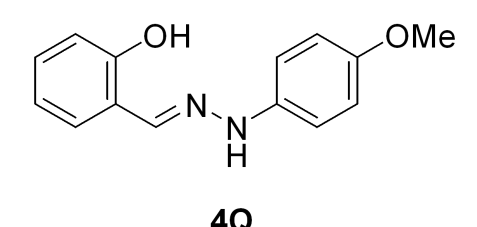

(75 $\mathrm{MHz}$ in $\mathrm{CHCl}_{3}$ )
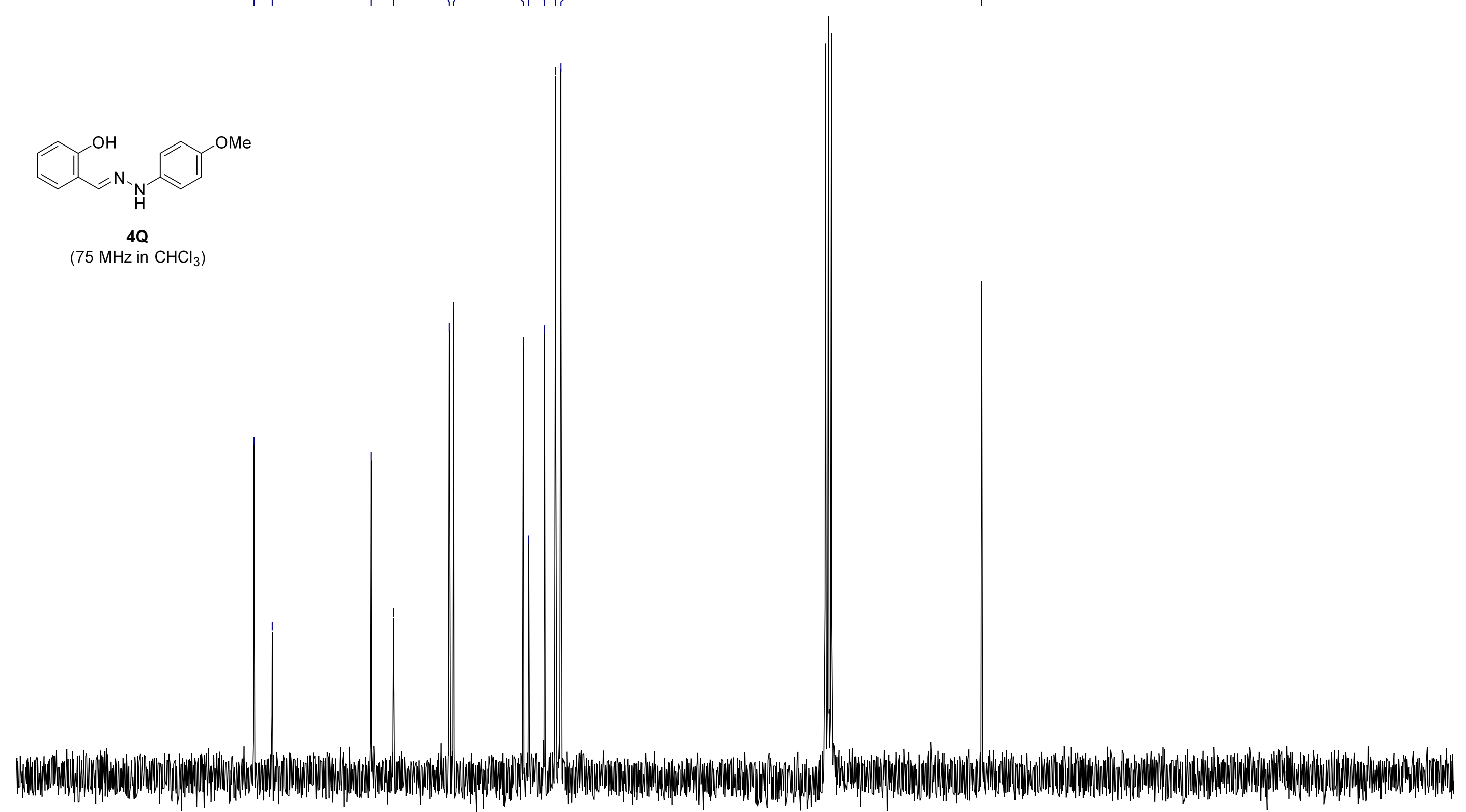


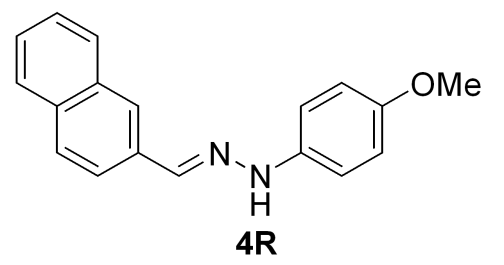

(300 MHz in DMSO- $d_{6}$ )

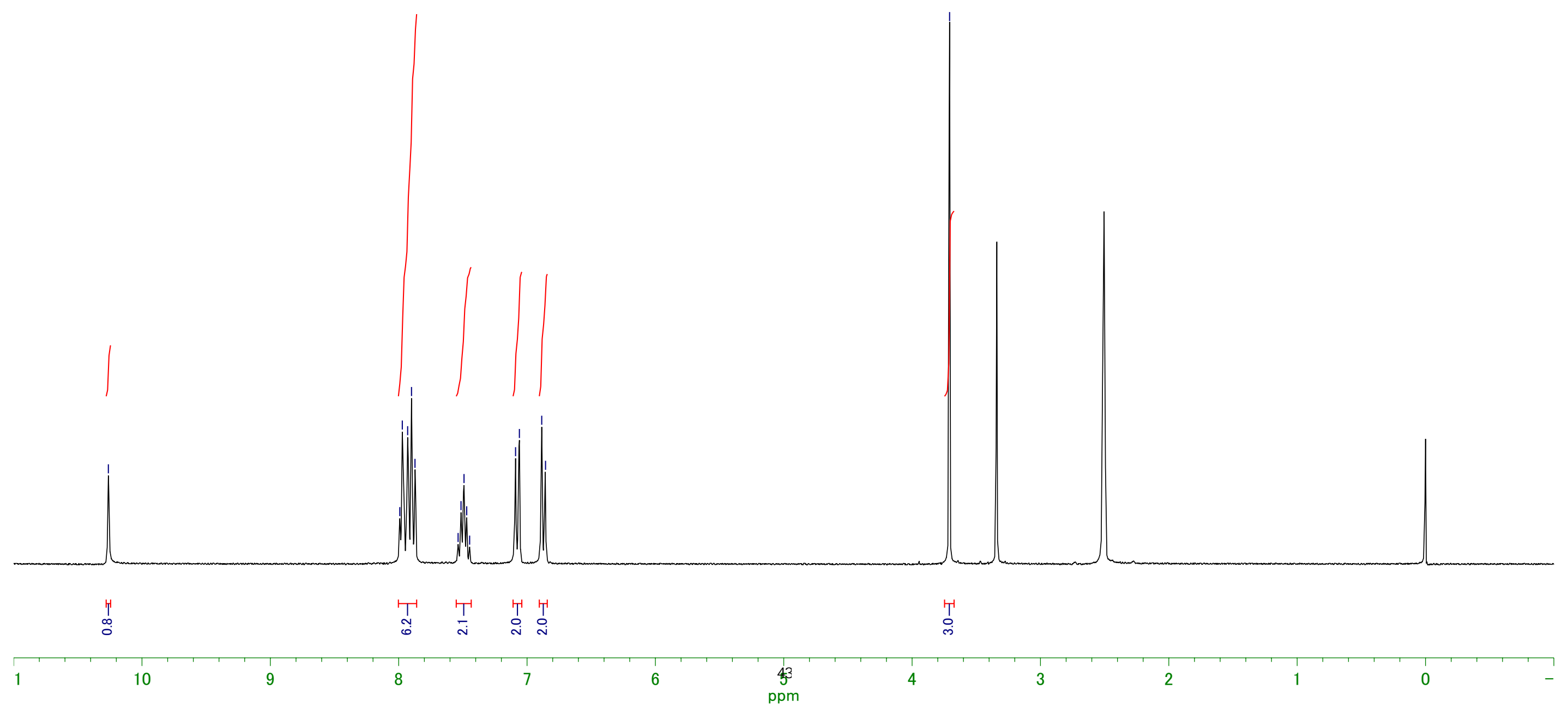




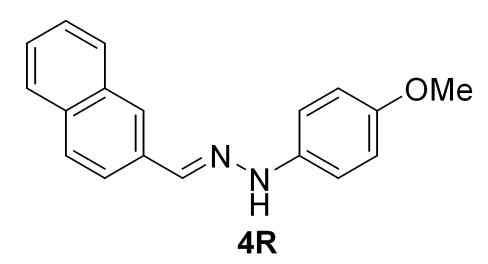

(75 MHz in DMSO- $d_{6}$ )

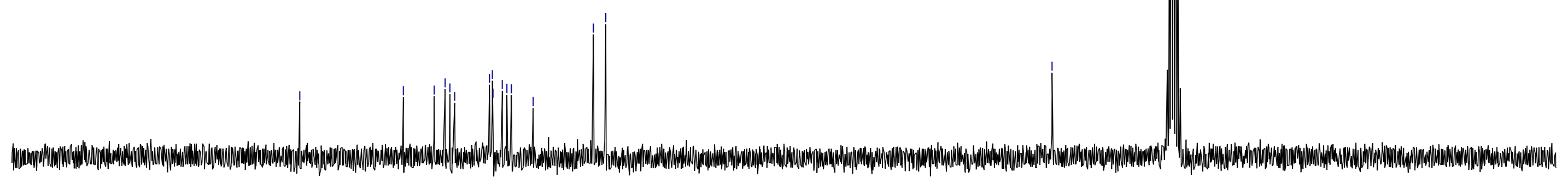




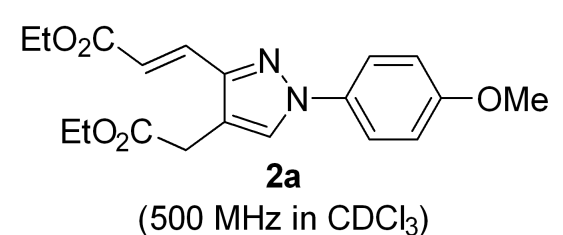

$\left(500 \mathrm{MHz}\right.$ in $\mathrm{CDCl}_{3}$ )
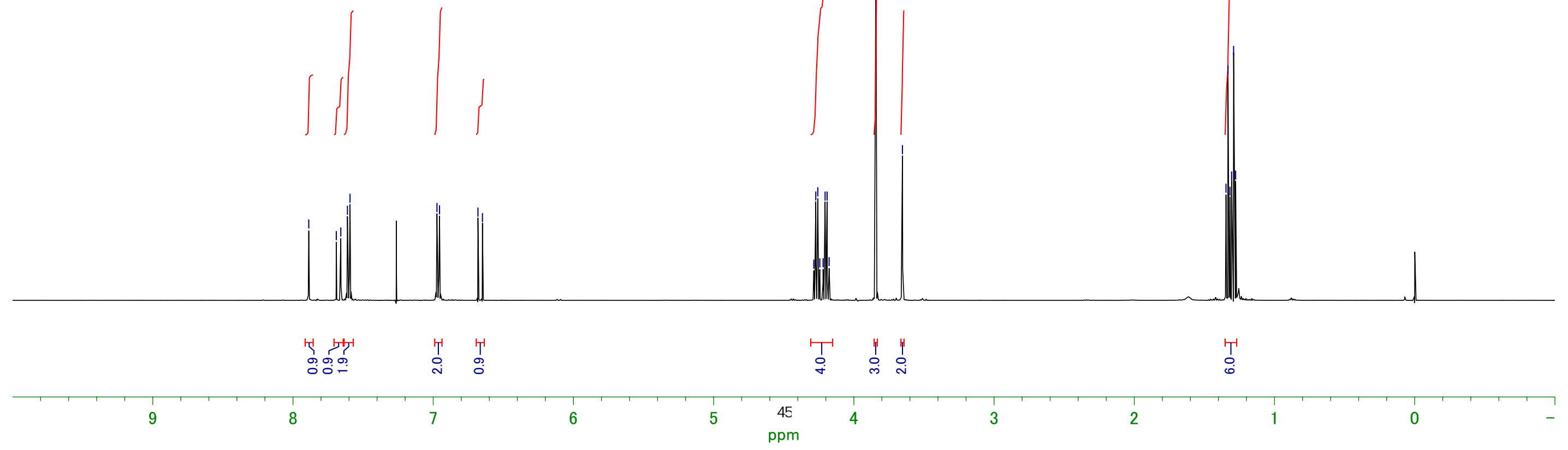


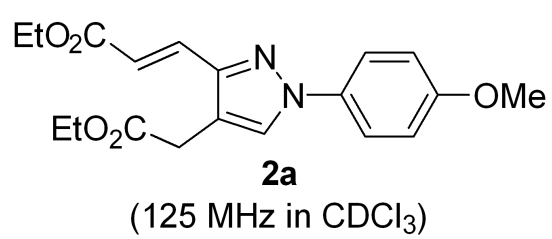



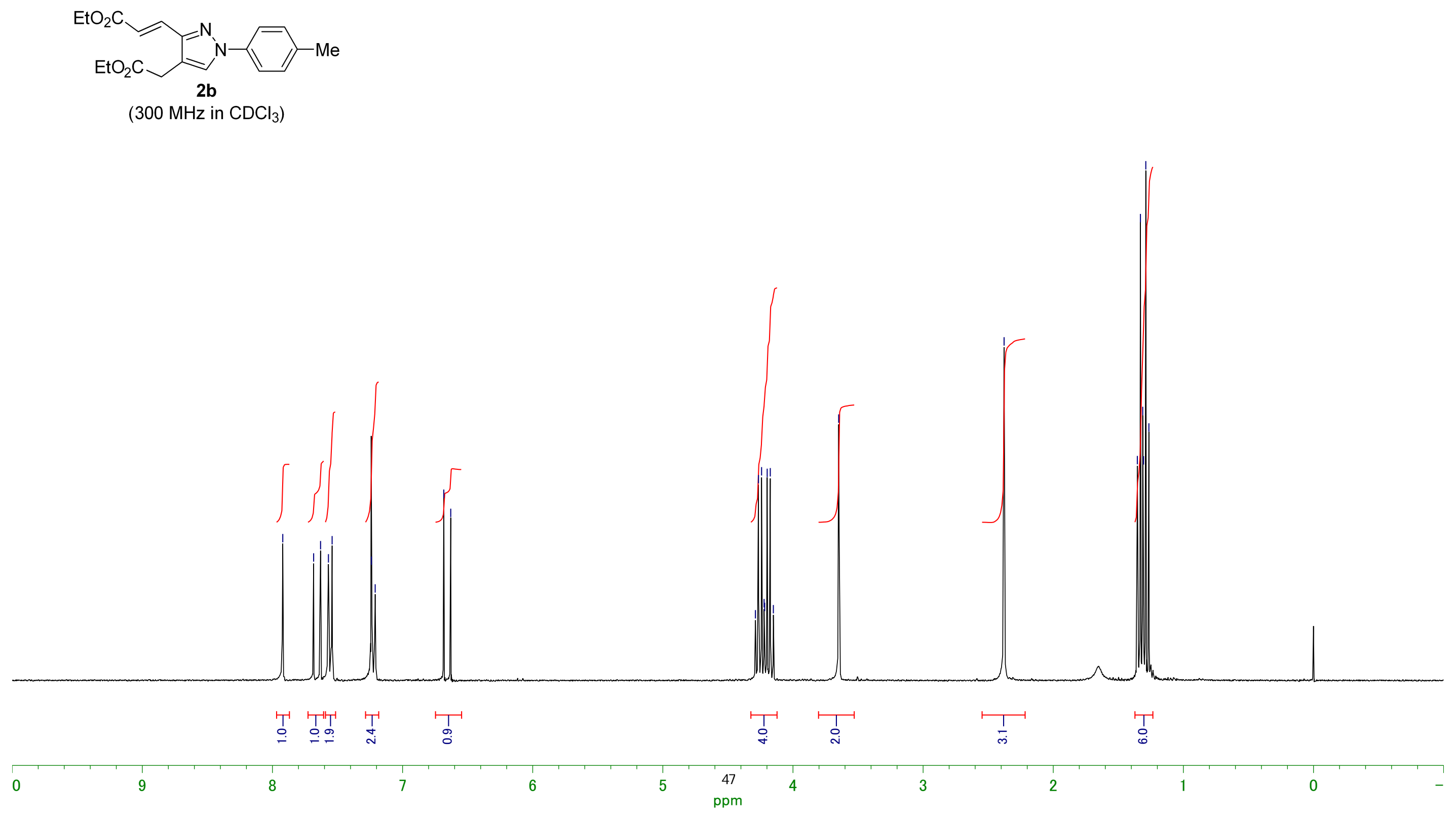


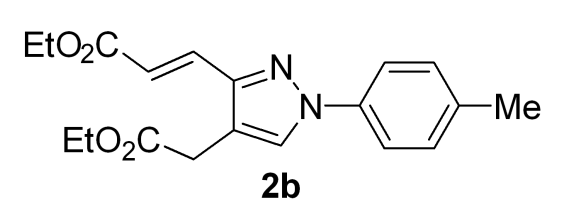

(75 $\mathrm{MHz}$ in $\mathrm{CDCl}_{3}$ ) 
$\mathrm{EtO}_{2} \mathrm{C}$<smiles>CCOC(=O)CCc1cn(-c2ccc(C(C)(C)C)cc2)nc1/C=C/C(=O)OCc1ccccc1</smiles>

(300 MHz in $\mathrm{CDCl}_{3}$ )
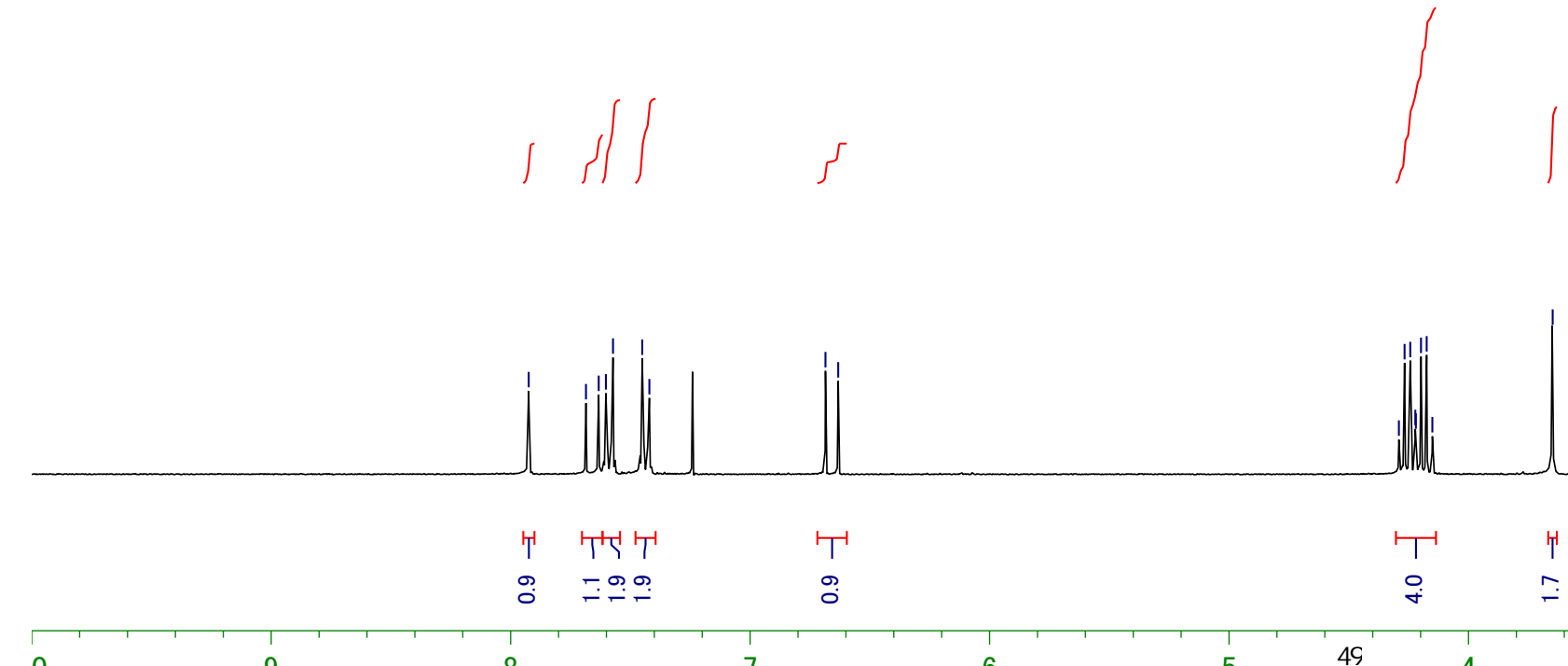

$\begin{array}{lll}T & T+1 \\ 8 & \\ 8 & \end{array}$

\begin{tabular}{l}
1 \\
0 \\
0 \\
\hline
\end{tabular}

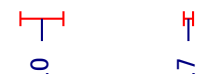

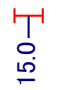




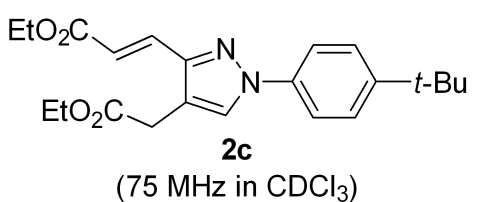

(75 $\mathrm{MHz}$ in $\mathrm{CDCl}_{3}$ ) 
$\left(300{\left.\mathrm{MHz} \text { in } \mathrm{CDCl}_{3}\right)}^{\mathrm{CtO}_{2} \mathrm{C}}\right.$

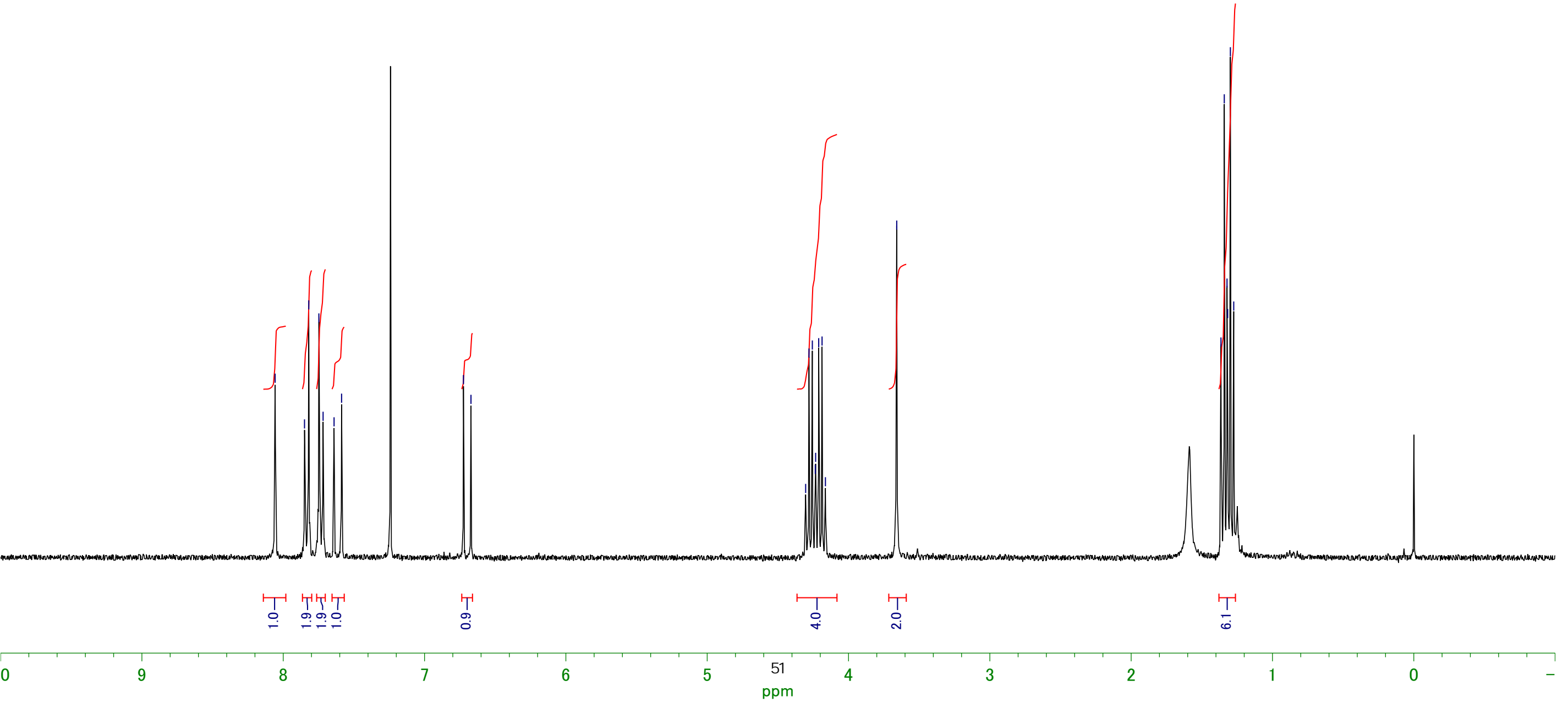




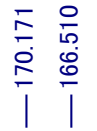

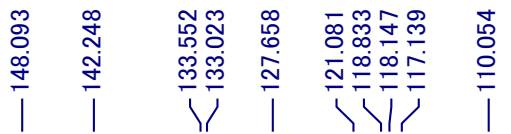

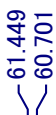

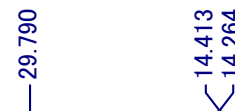

(75 $\left.\mathrm{MHz} \mathrm{in} \mathrm{CDCl}_{3}\right)$

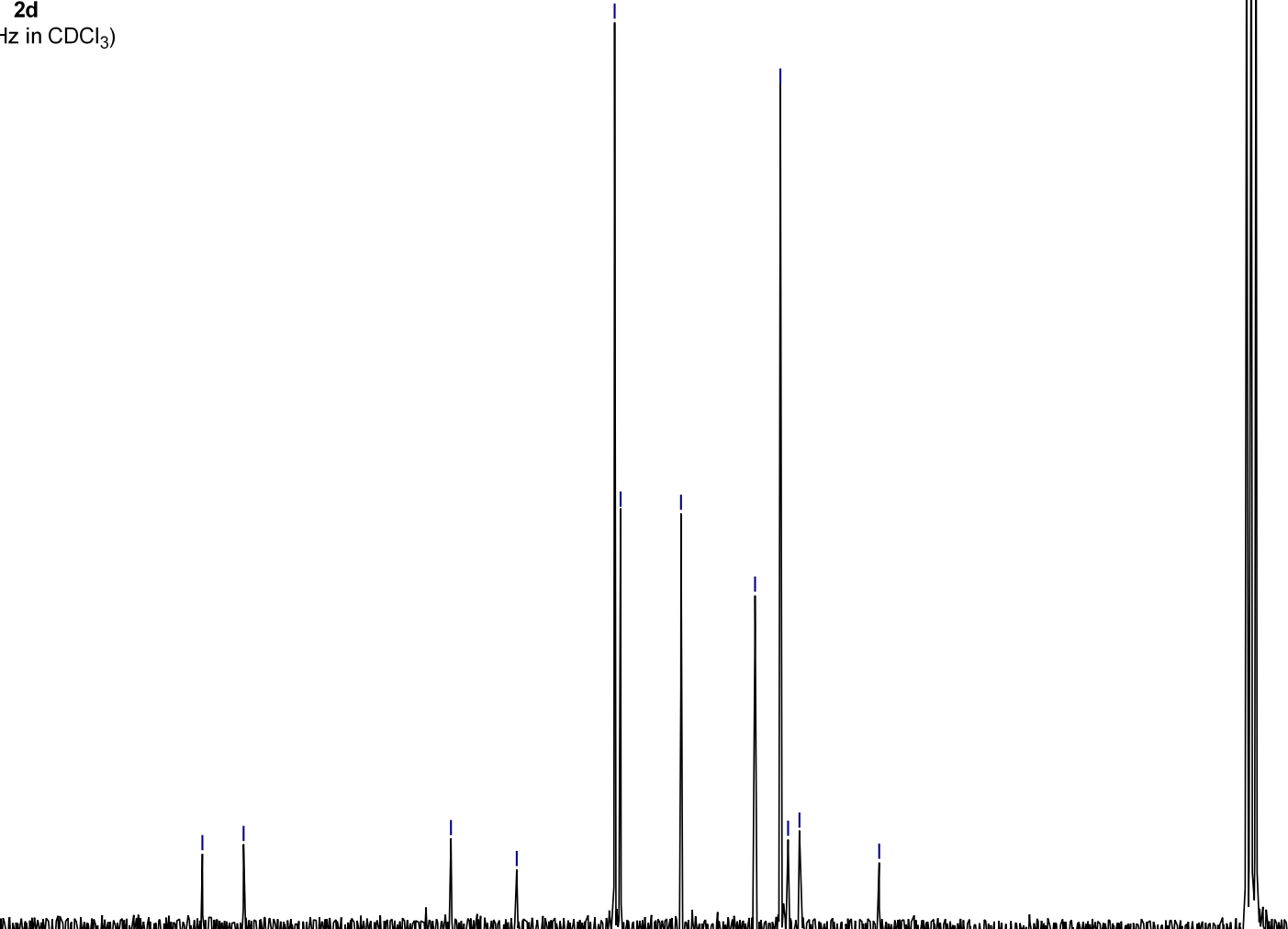

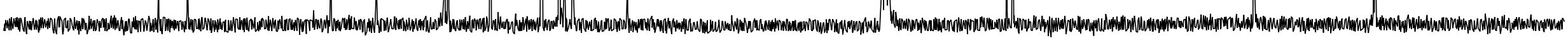


制

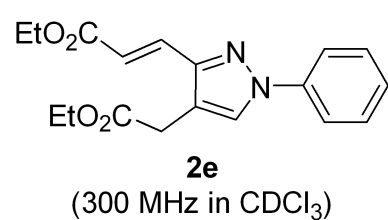

(300 $\mathrm{MHz}$ in $\mathrm{CDCl}_{3}$ )

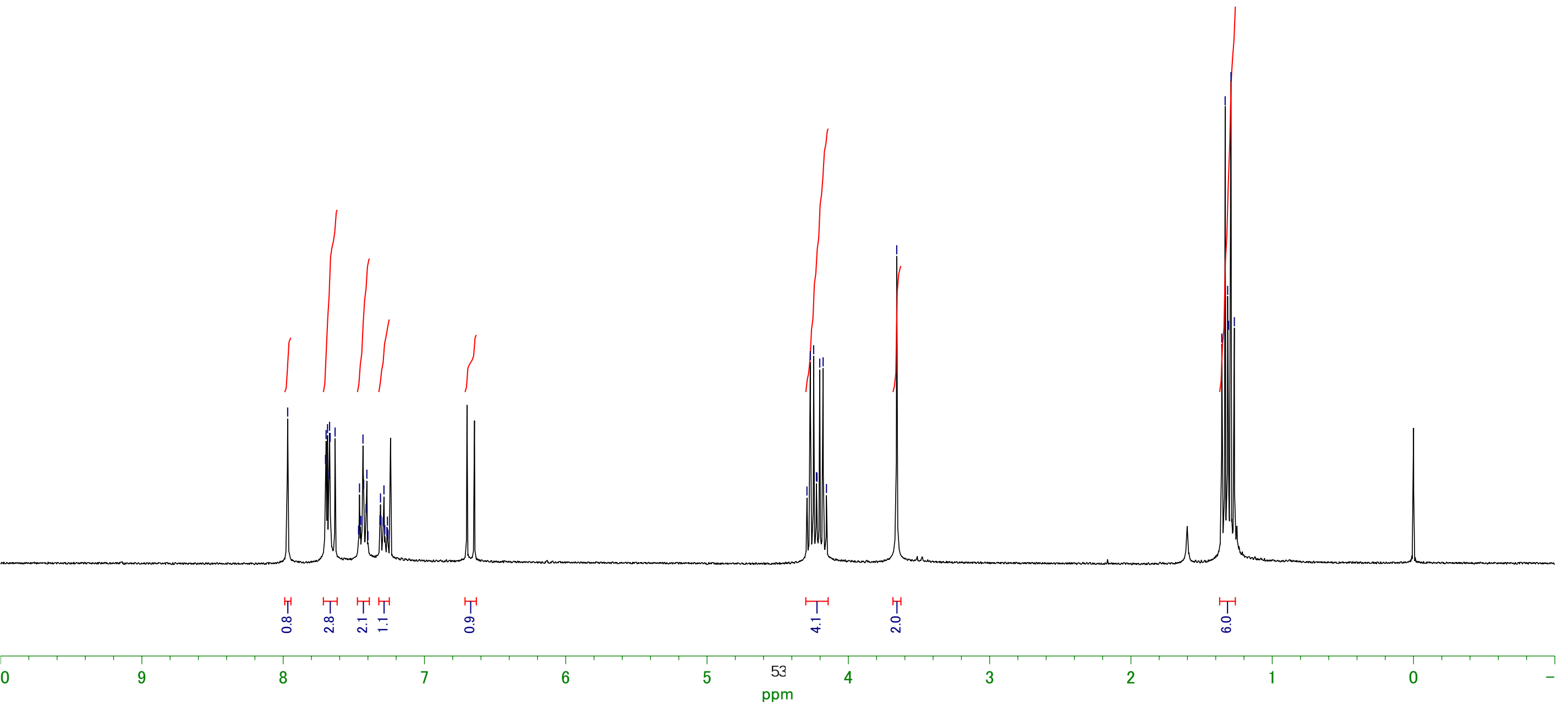




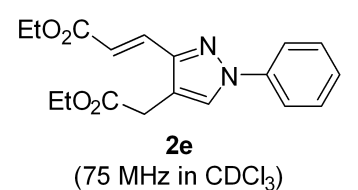


$\left(300 \mathrm{MHz}\right.$ in $\left.\mathrm{CDCl}_{3}\right)$

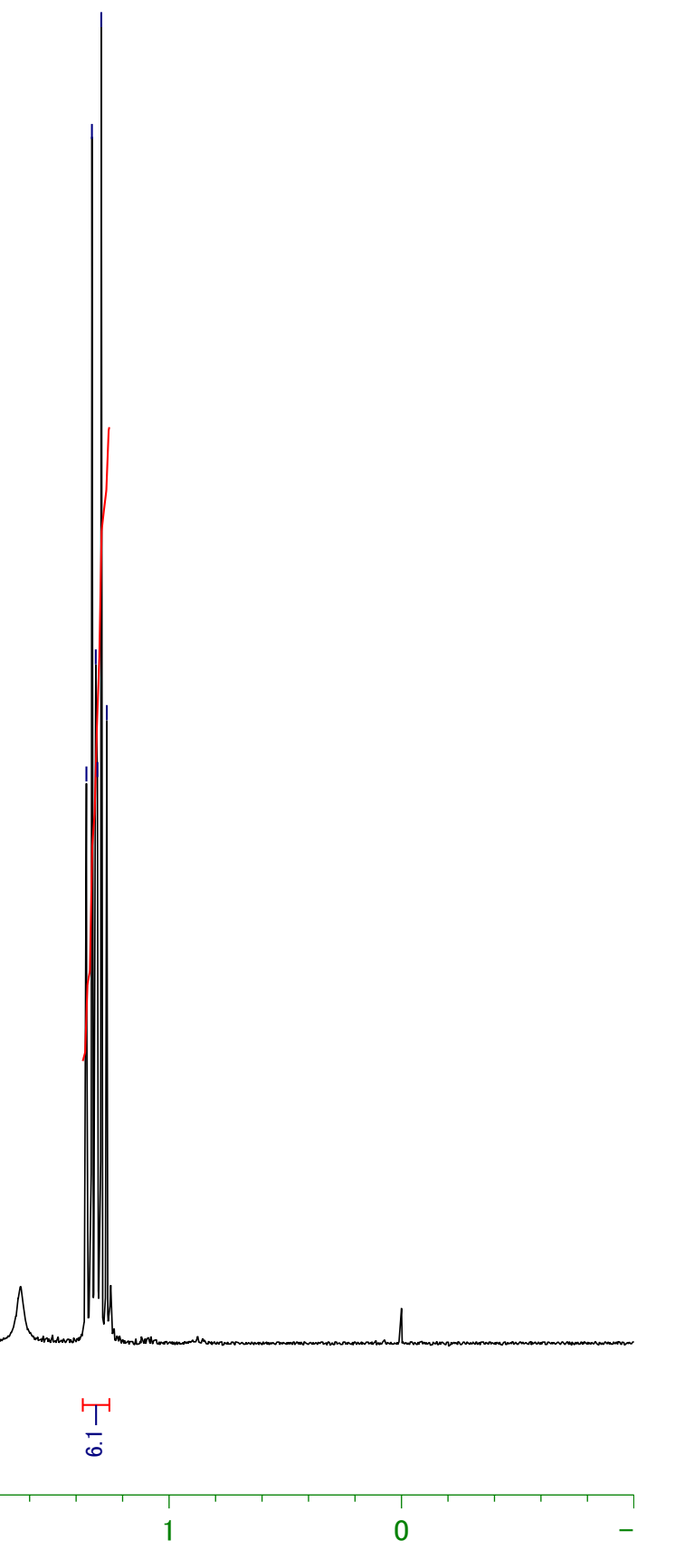




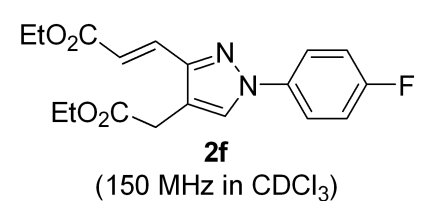

(150 MHz in $\mathrm{CDCl}_{3}$ ) 

$\left.(300 \mathrm{MHz} \text { in CDCl})_{3}\right)$
$\mathrm{EtO}_{2} \mathrm{C}$

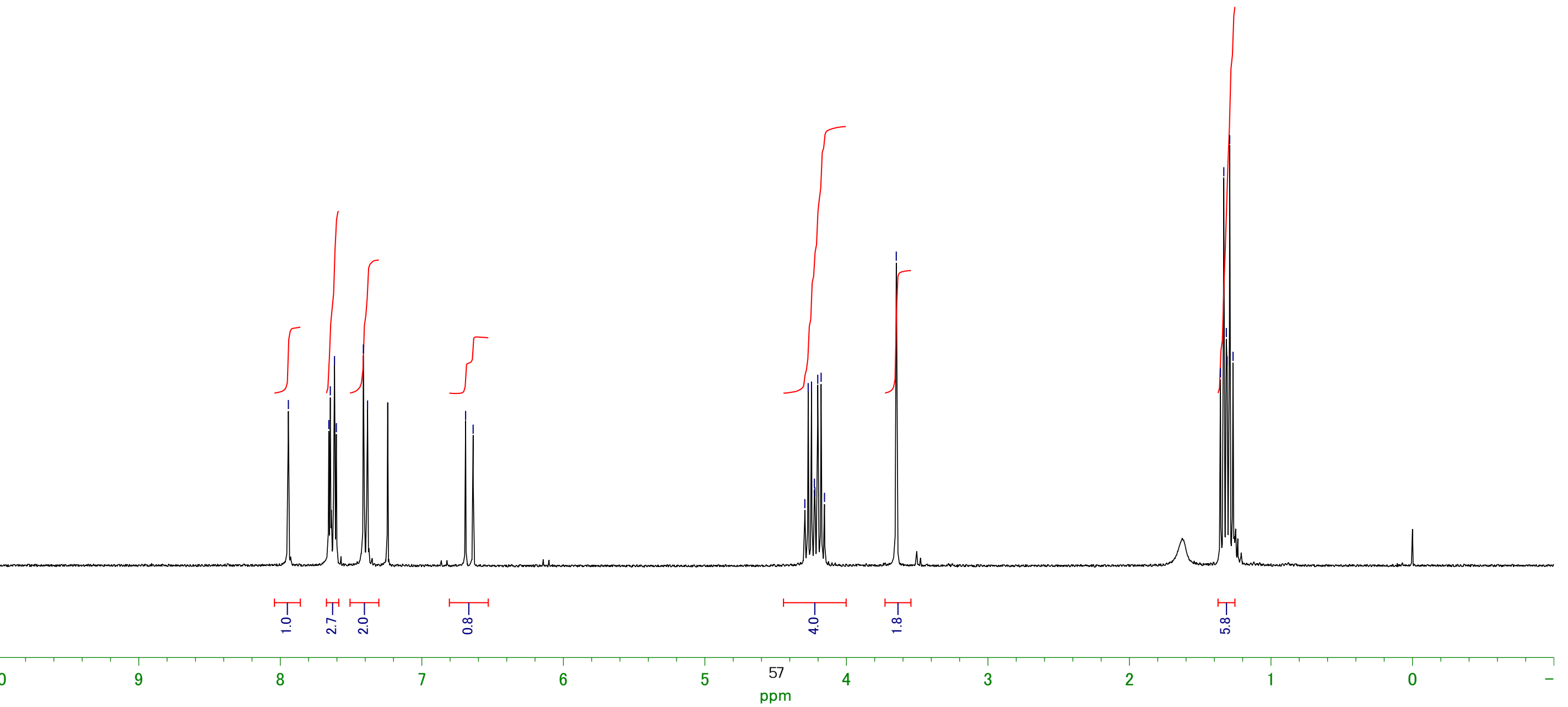




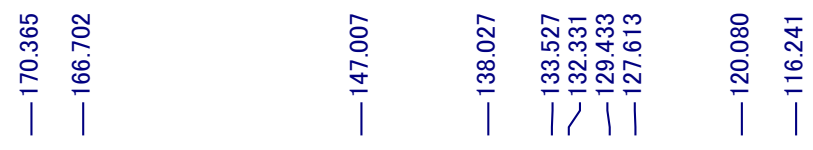

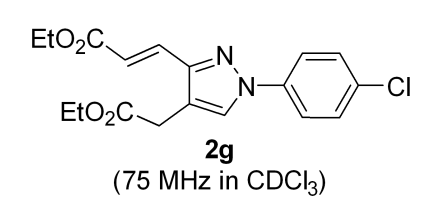




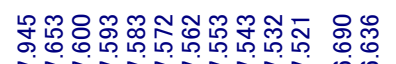

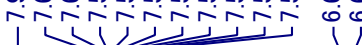

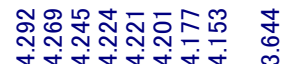

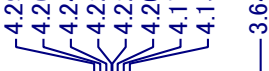

递然

-

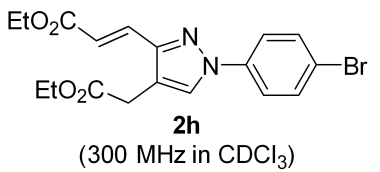

(300 $\mathrm{MHz}$ in $\mathrm{CDCl}_{3}$ )

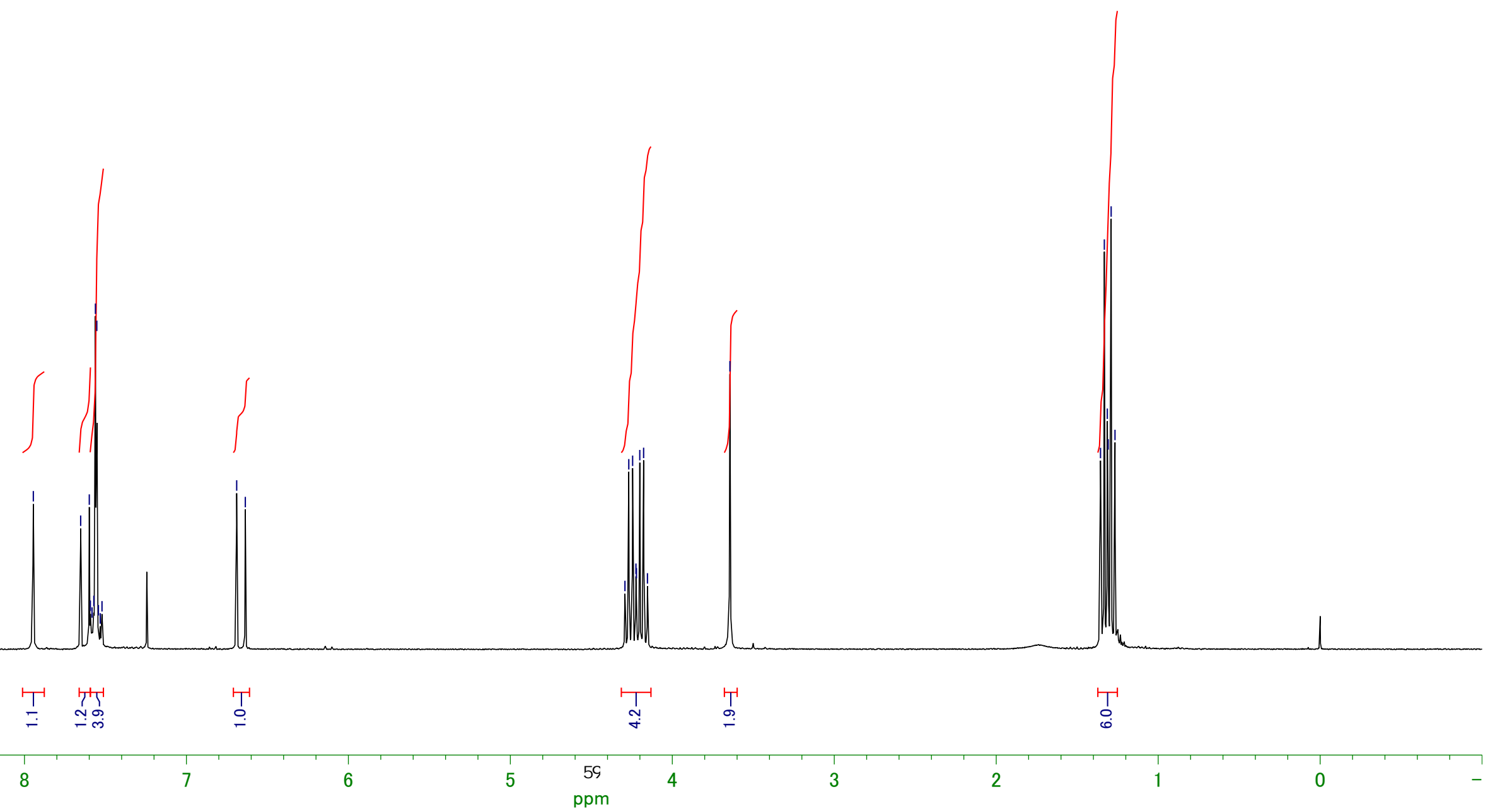




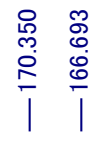
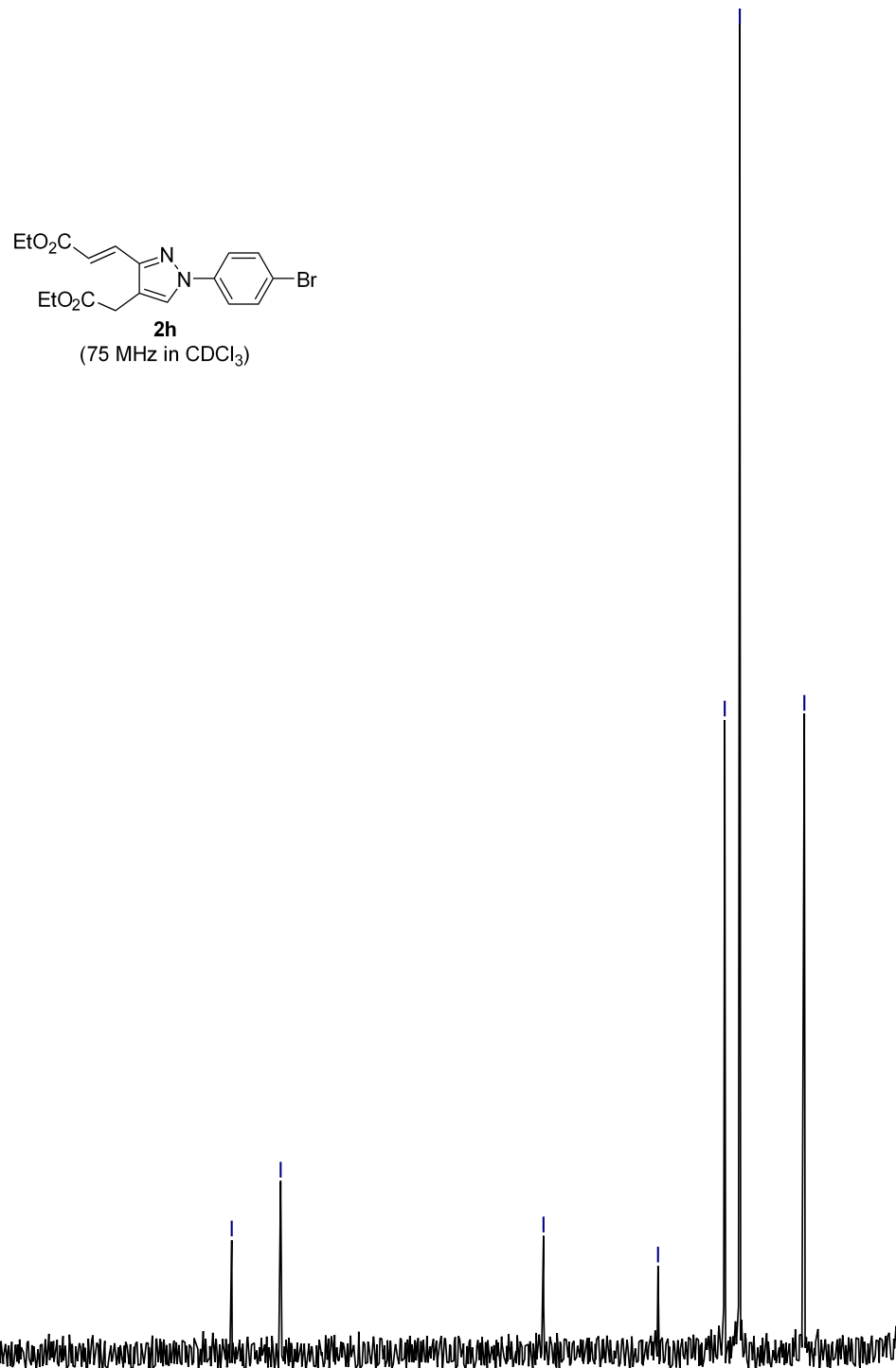


$\left(300 \mathrm{MHz}\right.$ in $\left.\mathrm{CDCl}_{3}\right)$

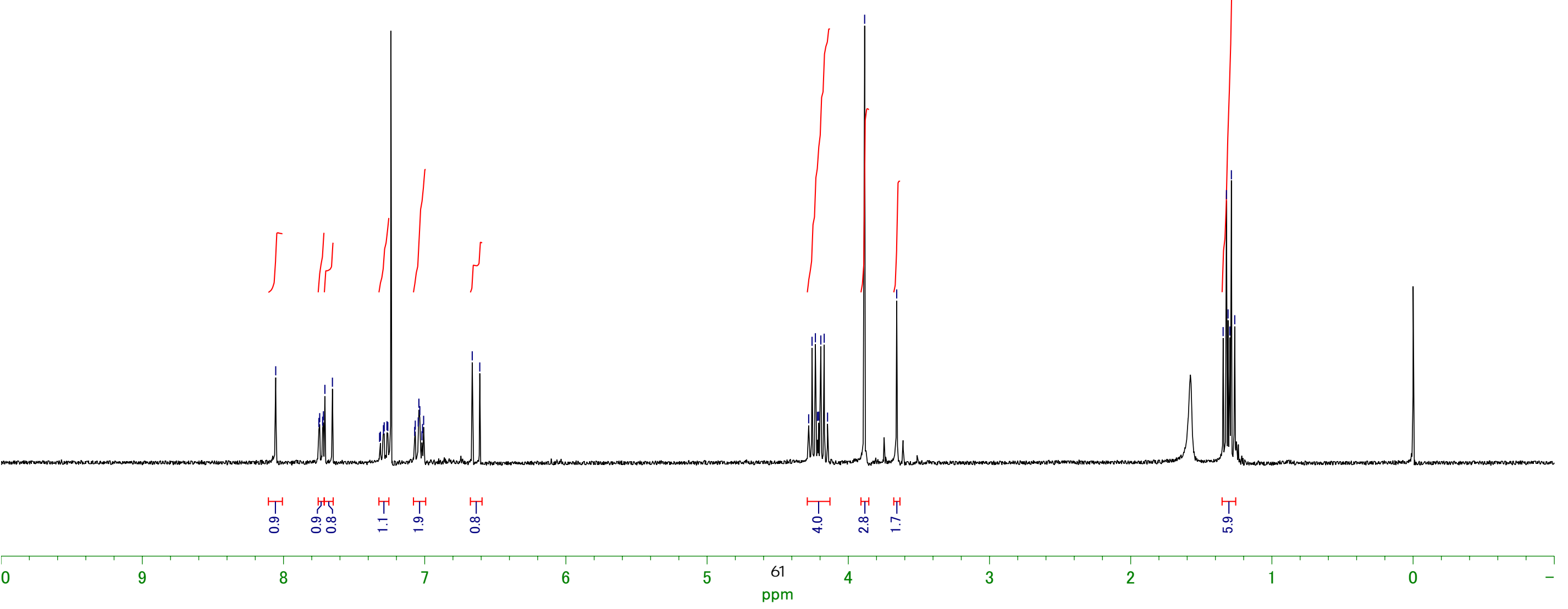



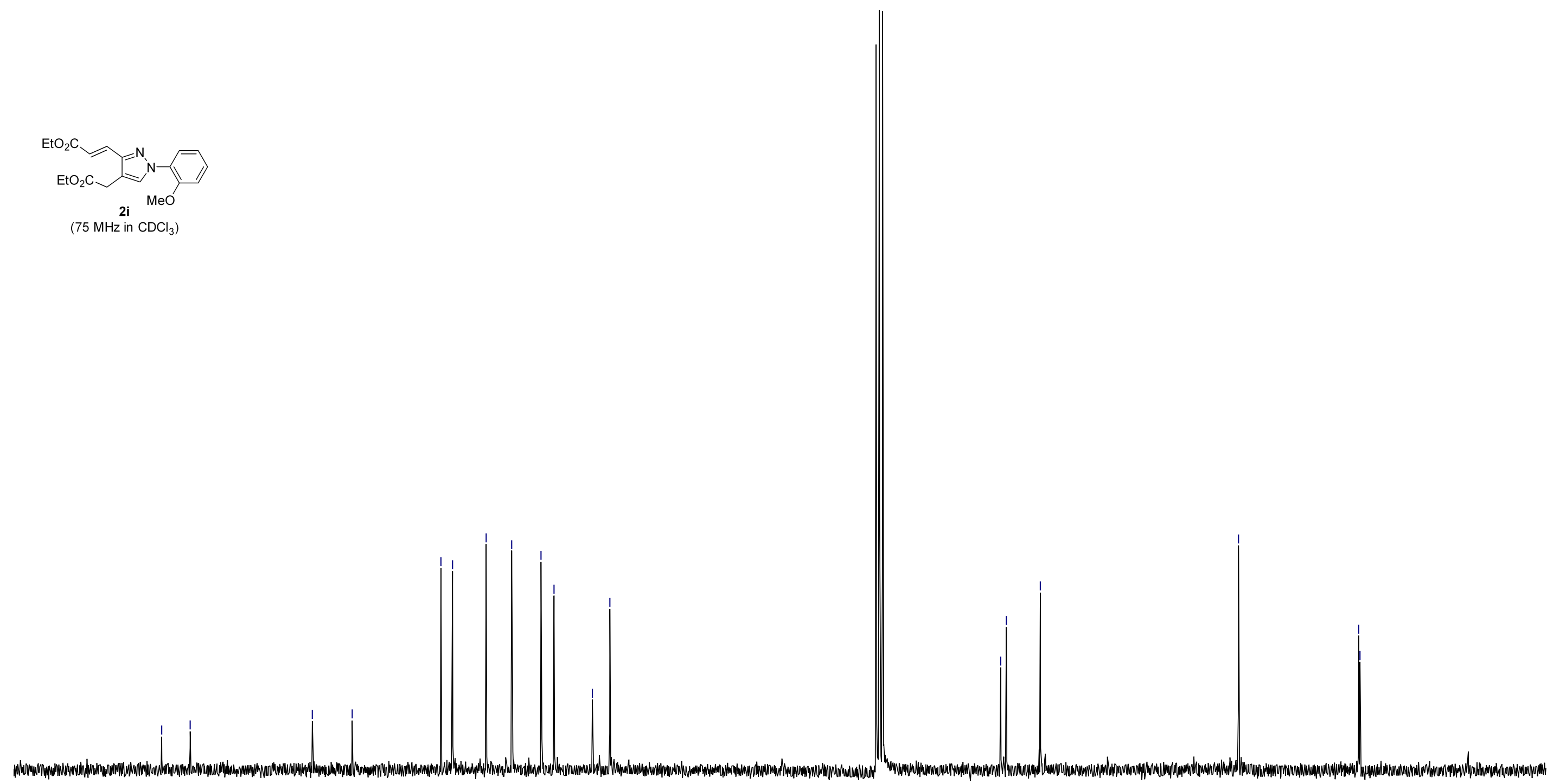
$\mathrm{MeO}_{2} \mathrm{C}=\mathrm{OMe}$

5jA
$\left(300 \mathrm{MHz}\right.$ in $\left.\mathrm{CDCl}_{3}\right)$

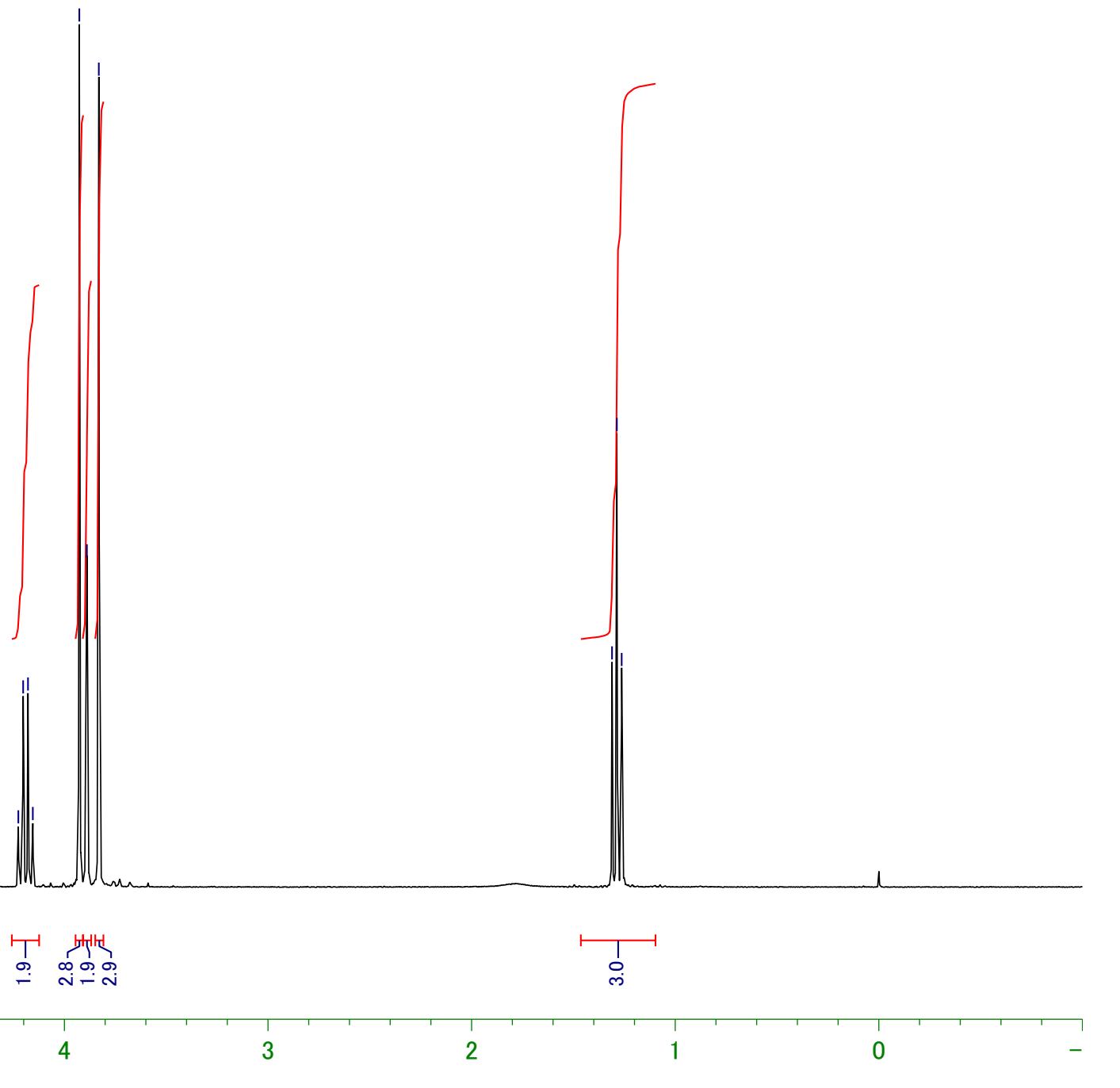




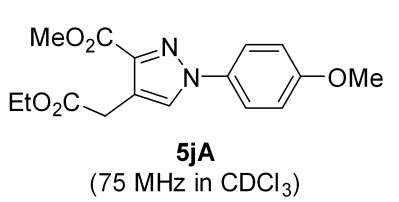

5jA
$\left(75 \mathrm{MHz}\right.$ in $\left.\mathrm{CDCl}_{3}\right)$

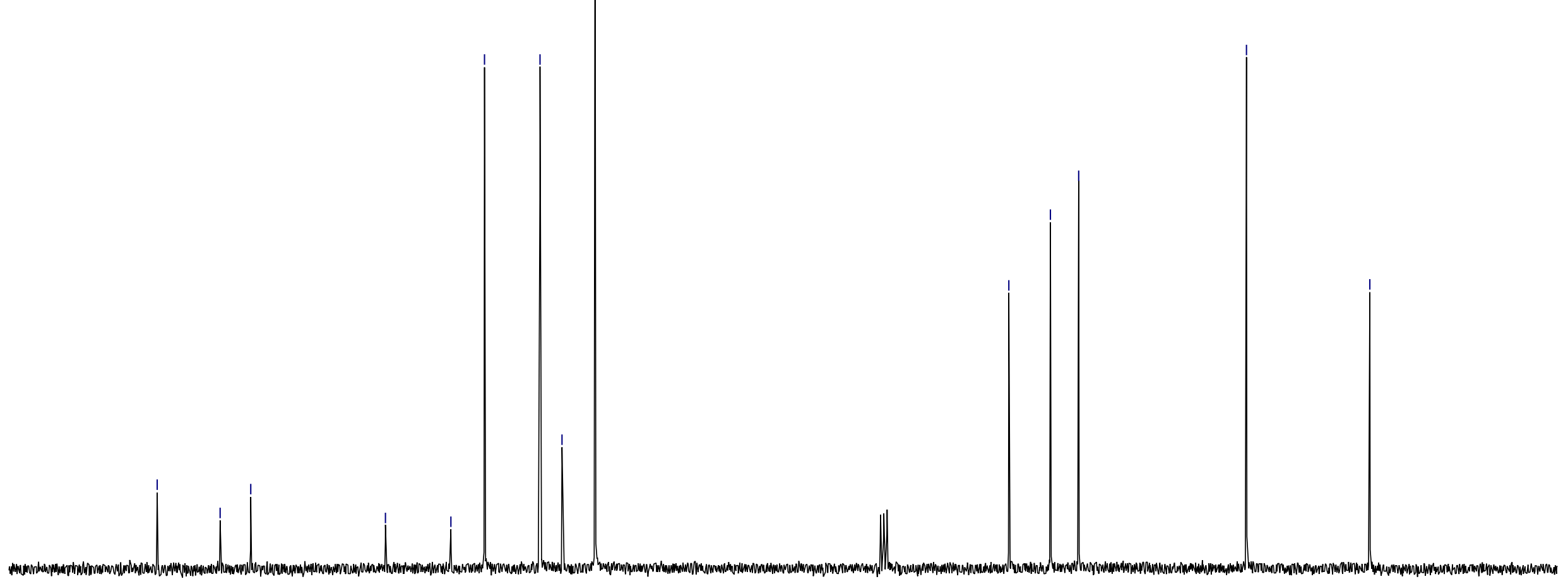


$\mathrm{MeO}_{2} \mathrm{C}=\mathrm{N}$

$5 \mathrm{jB}$

( $300 \mathrm{MHz}$ in $\mathrm{CDCl}_{3}$ )

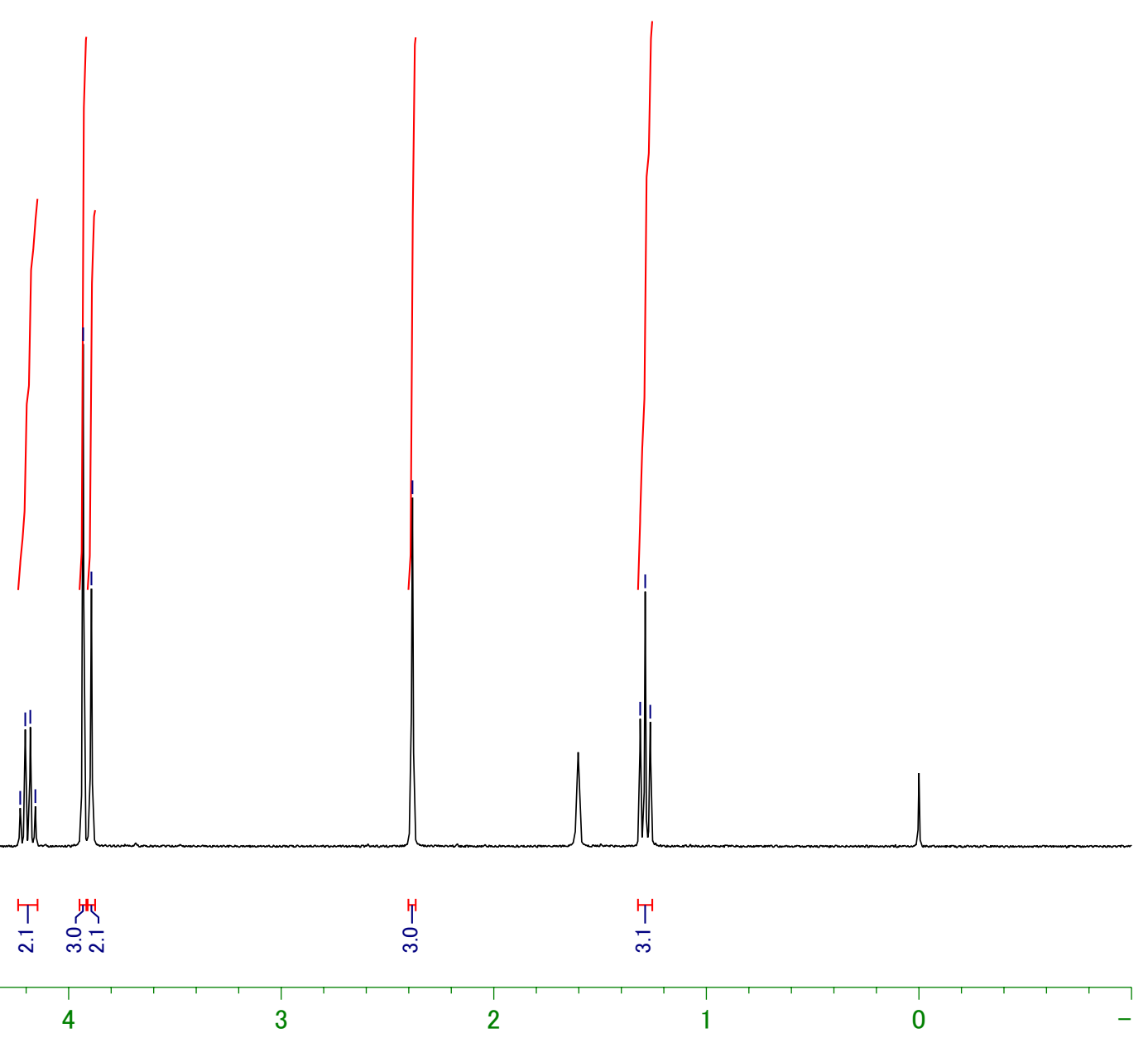




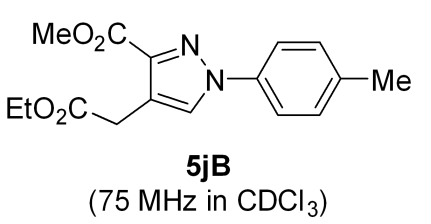

$\left(75 \mathrm{MHz}\right.$ in $\left.\mathrm{CDCl}_{3}\right)$ 
(300 $\mathrm{MHz}$ in $\left.\mathrm{CDCl}_{3}\right)$

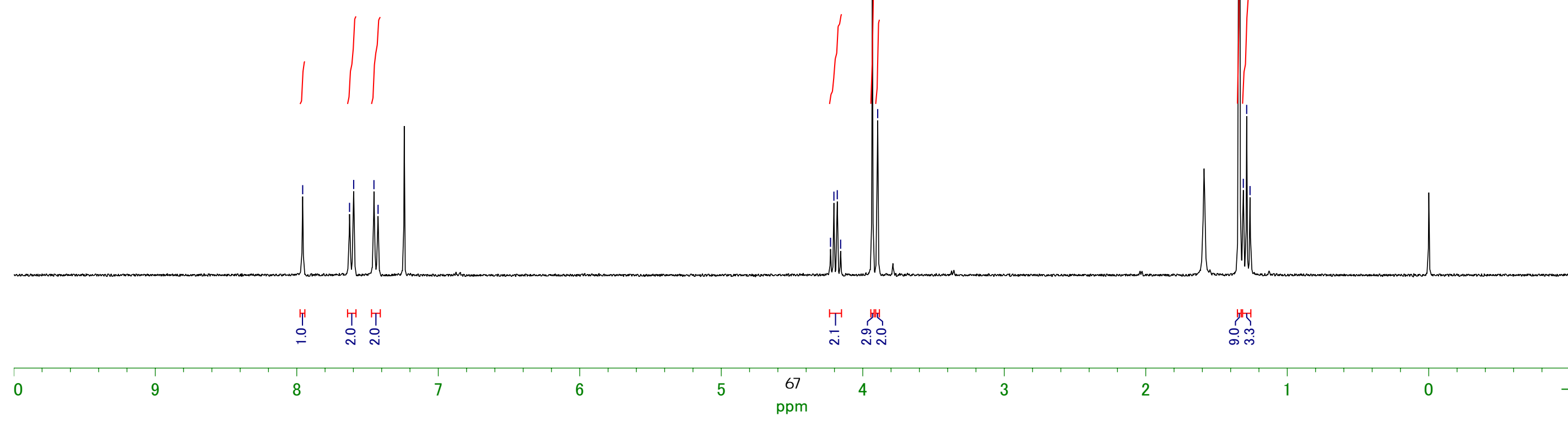




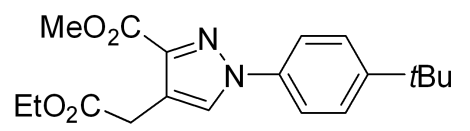

$5 \mathrm{jC}$

(75 $\mathrm{MHz}$ in $\mathrm{CDCl}_{3}$ )

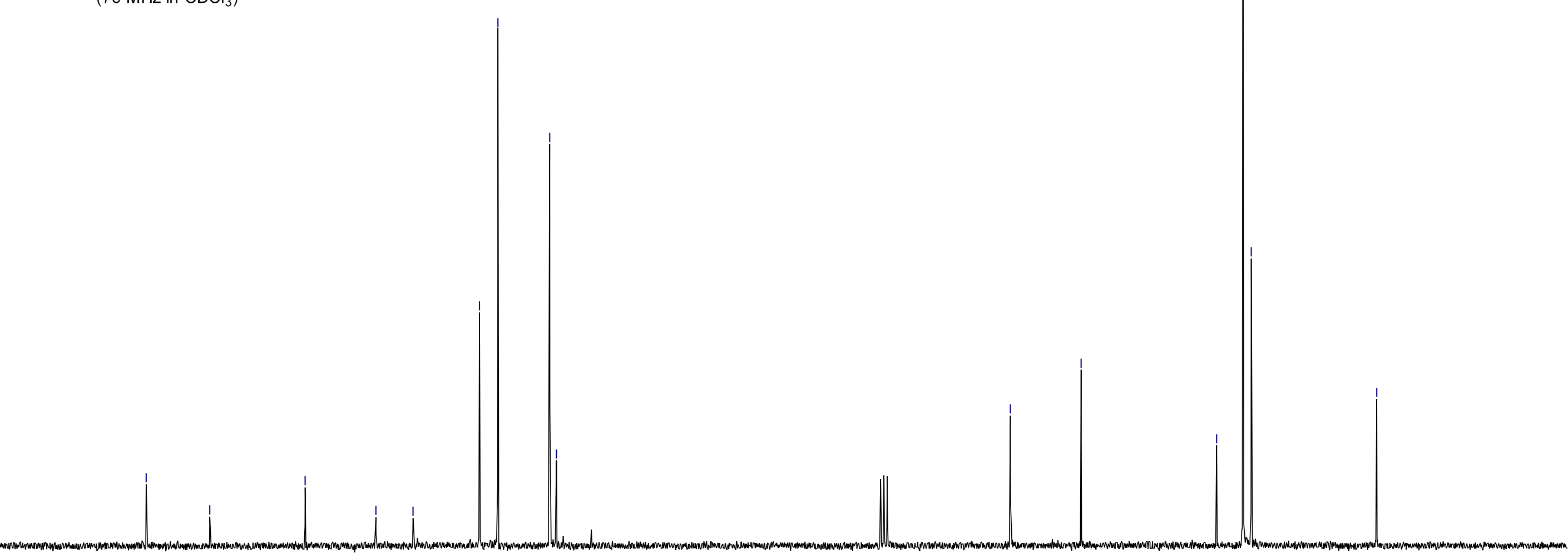



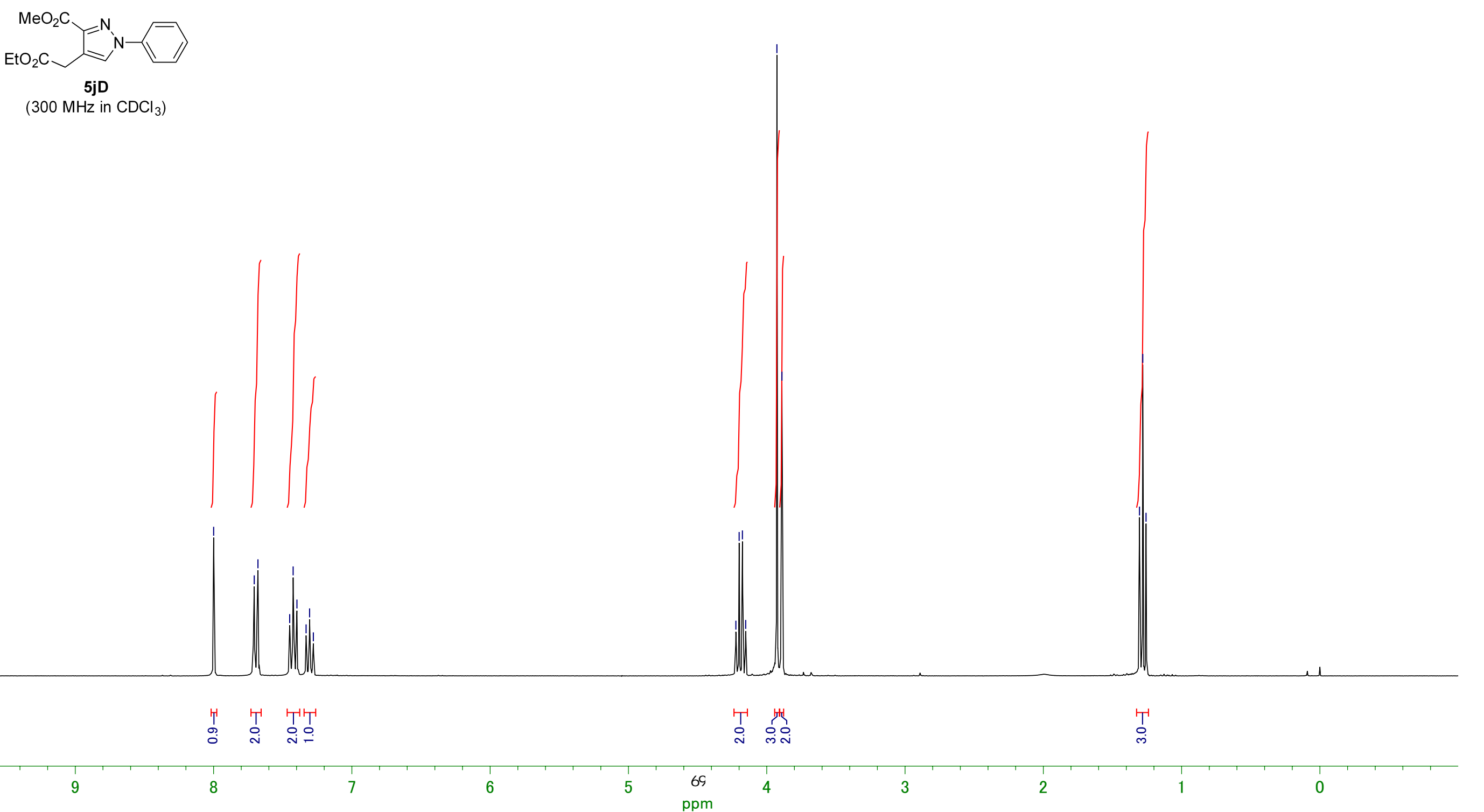

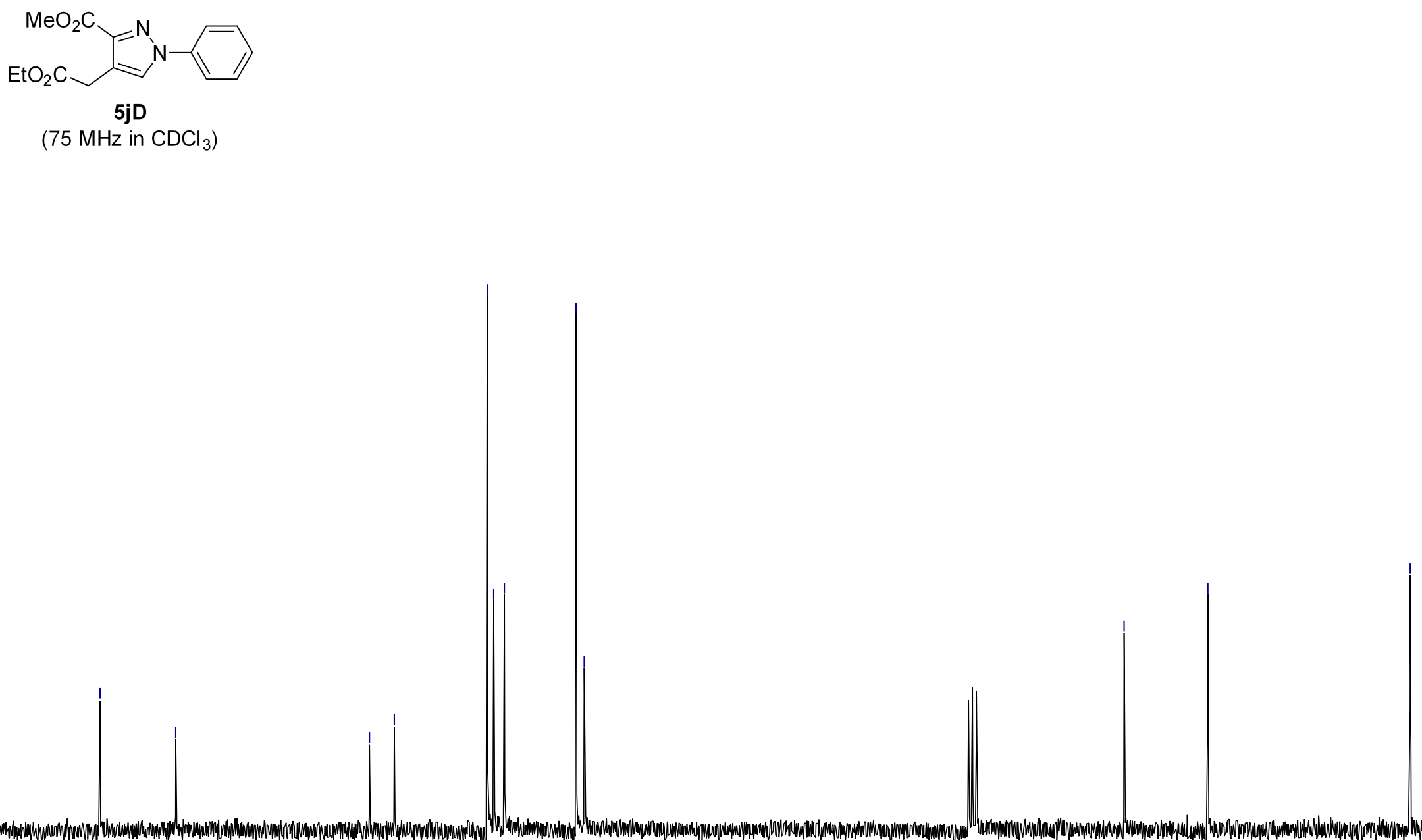

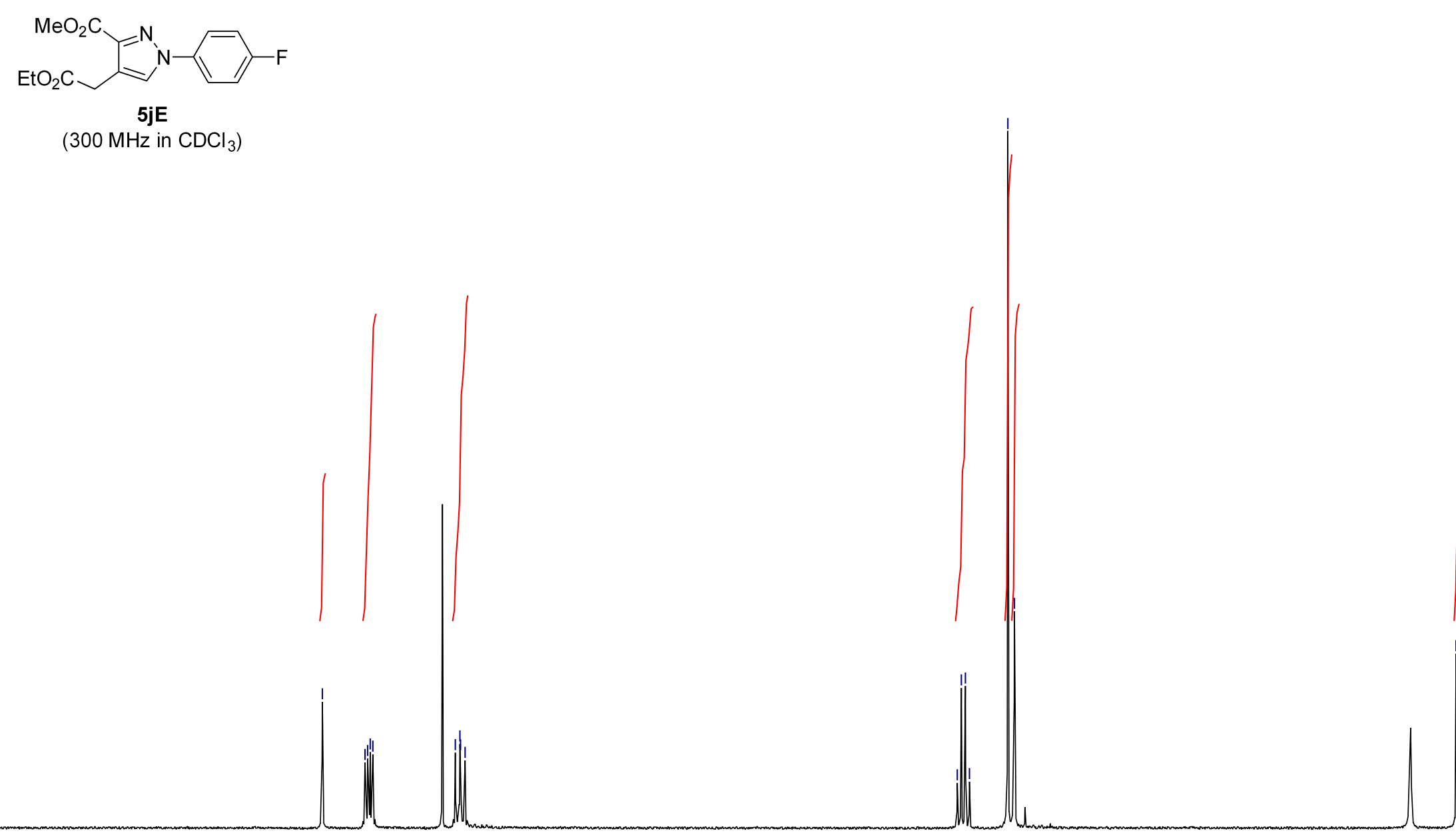

雚

'T

T' 


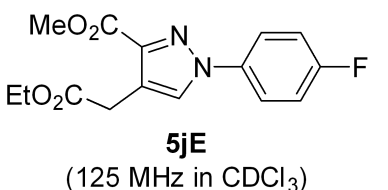

(125 $\mathrm{MHz}$ in $\mathrm{CDCl}_{3}$ )

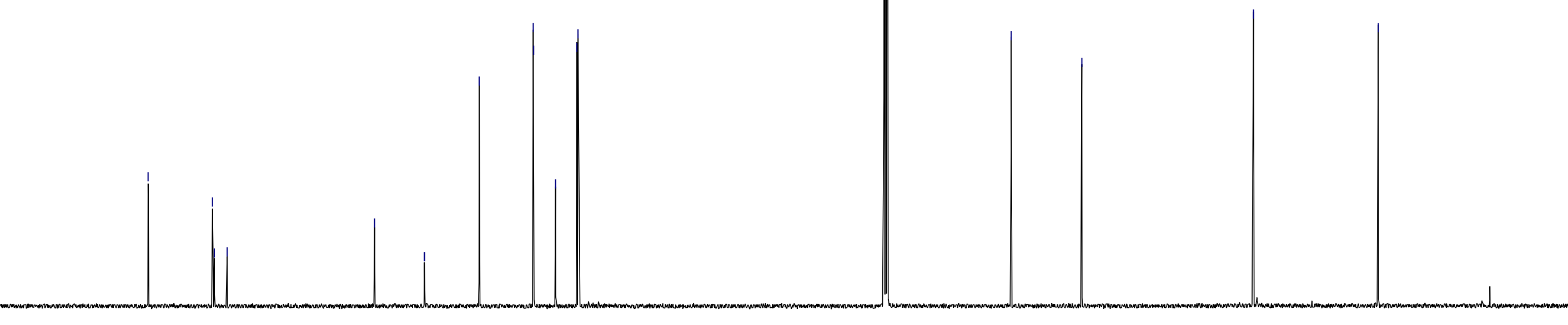


$\left(300 \mathrm{MHz}\right.$ in $\left.\mathrm{CDCl}_{3}\right)$

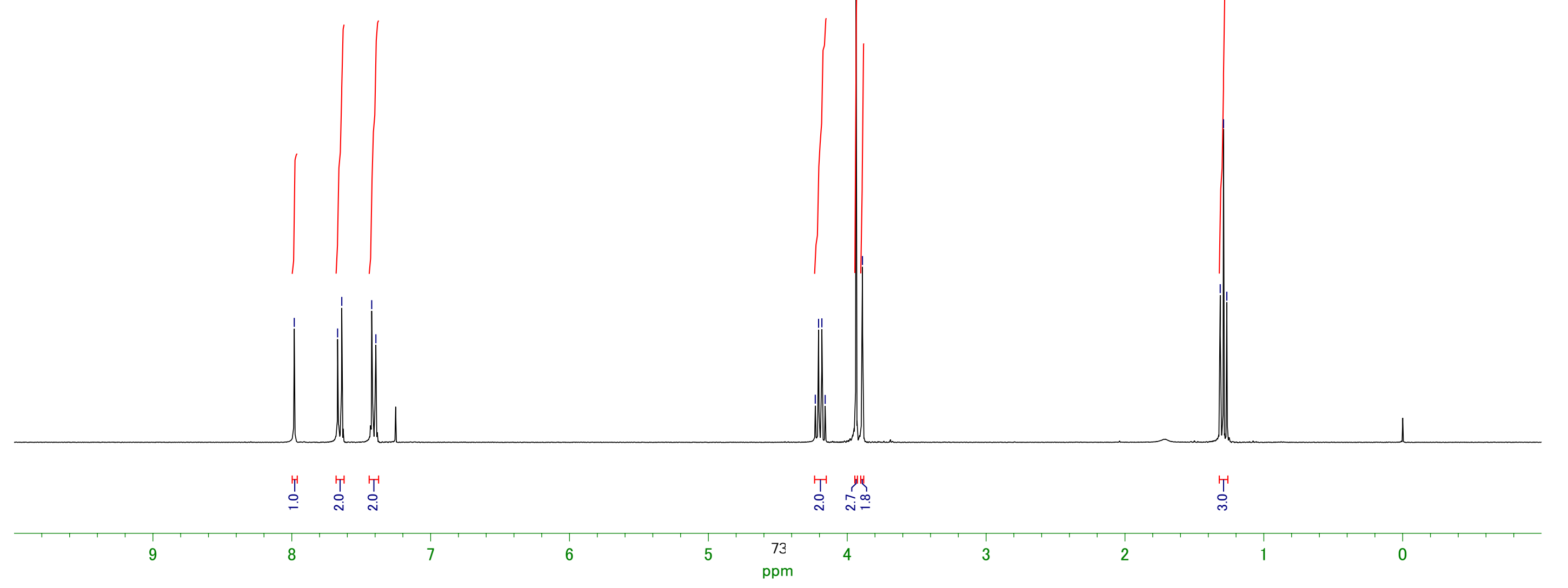




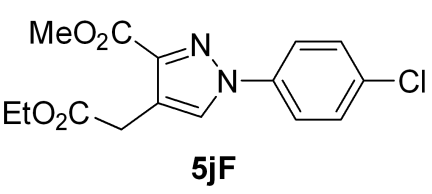

$\left(75 \mathrm{MHz}\right.$ in $\left.\mathrm{CDCl}_{3}\right)$ 
$\mathrm{MeO}_{2} \mathrm{C}=\mathrm{N}$

5jG

(300 $\mathrm{MHz}$ in $\mathrm{CDCl}_{3}$ )

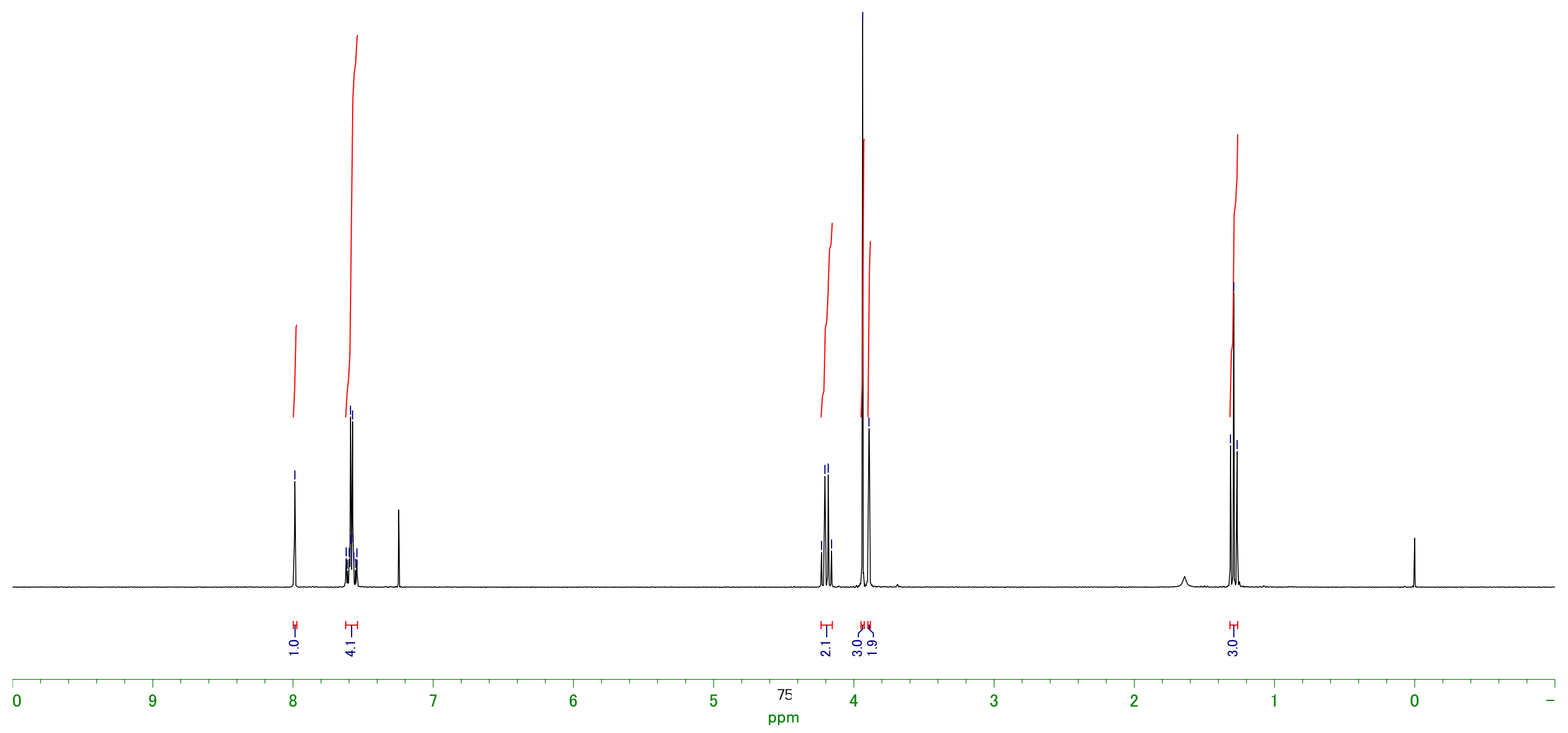




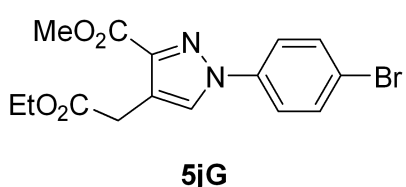

(75 $\mathrm{MHz}$ in $\mathrm{CDCl}_{3}$ ) 


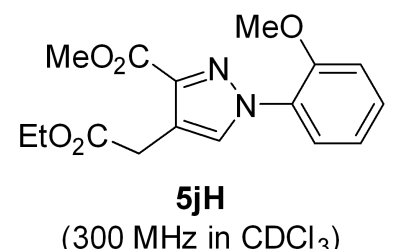

(300 $\mathrm{MHz}$ in $\mathrm{CDCl}_{3}$ )

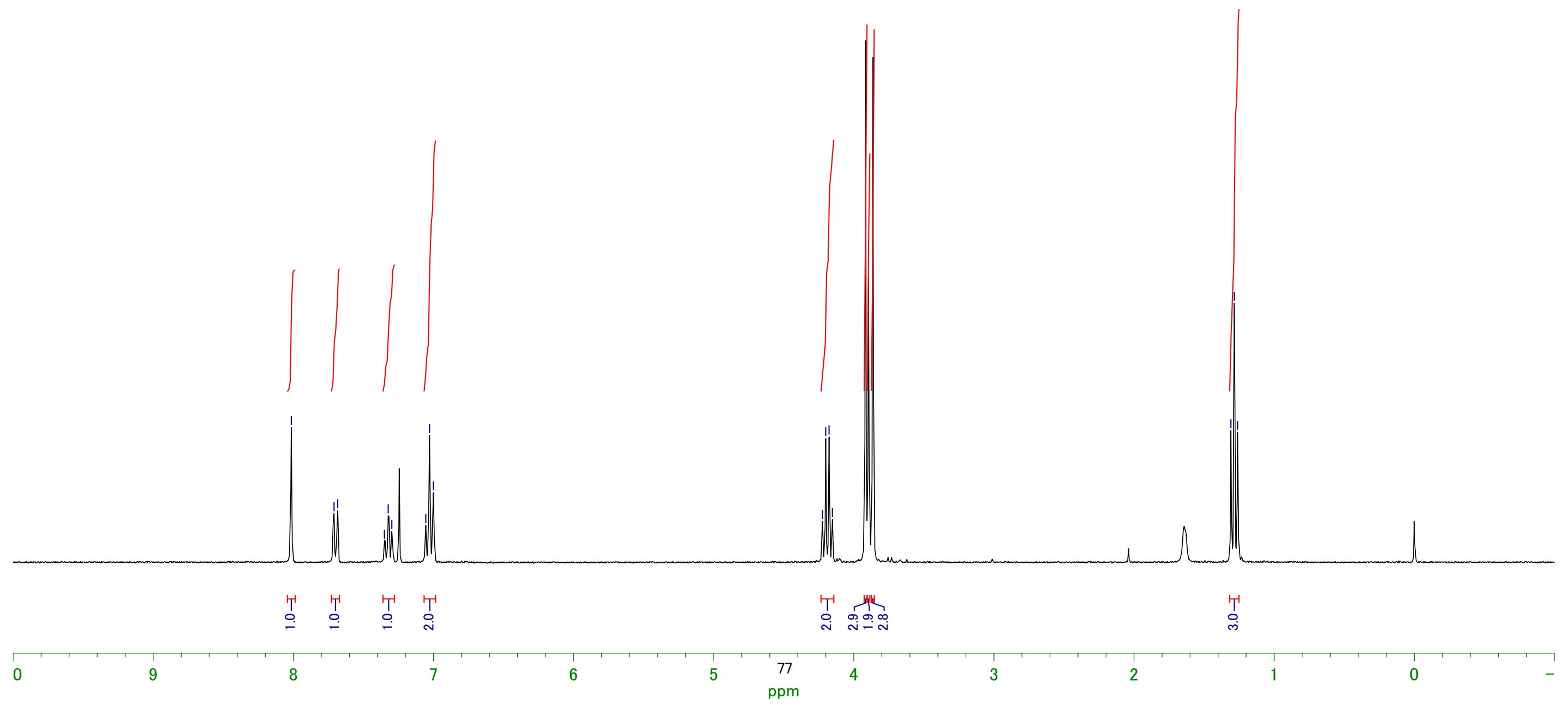




$$
\text { 旁 }
$$

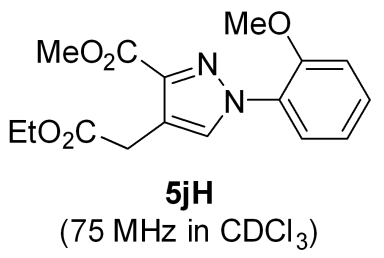




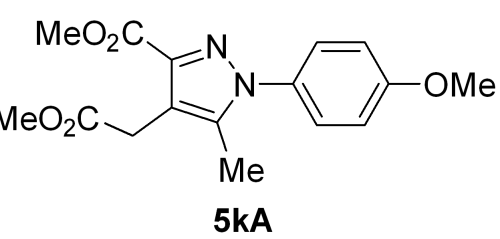

(300 $\mathrm{MHz}$ in $\mathrm{CDCl}_{3}$ )

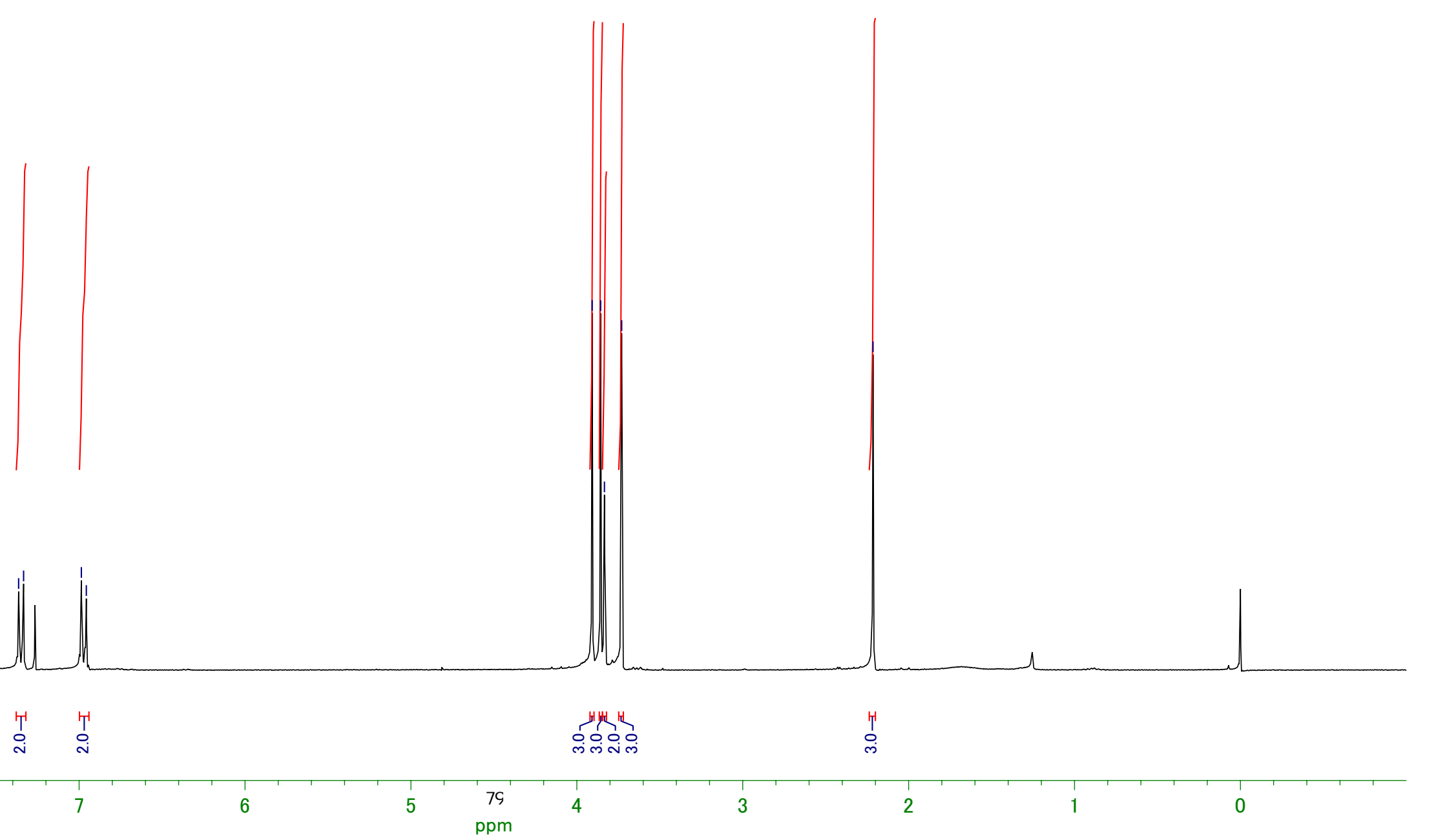




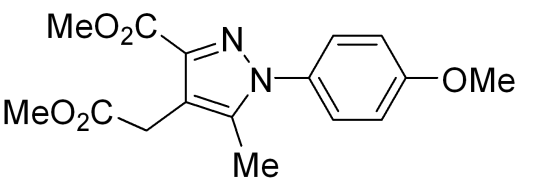

5kA

(75 $\mathrm{MHz}$ in $\mathrm{CDCl}_{3}$ )

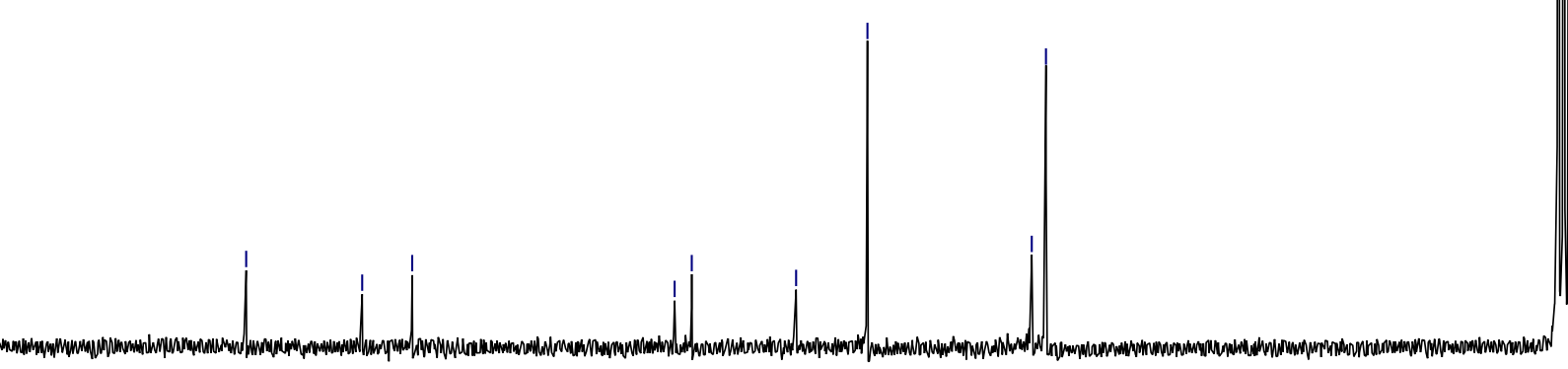



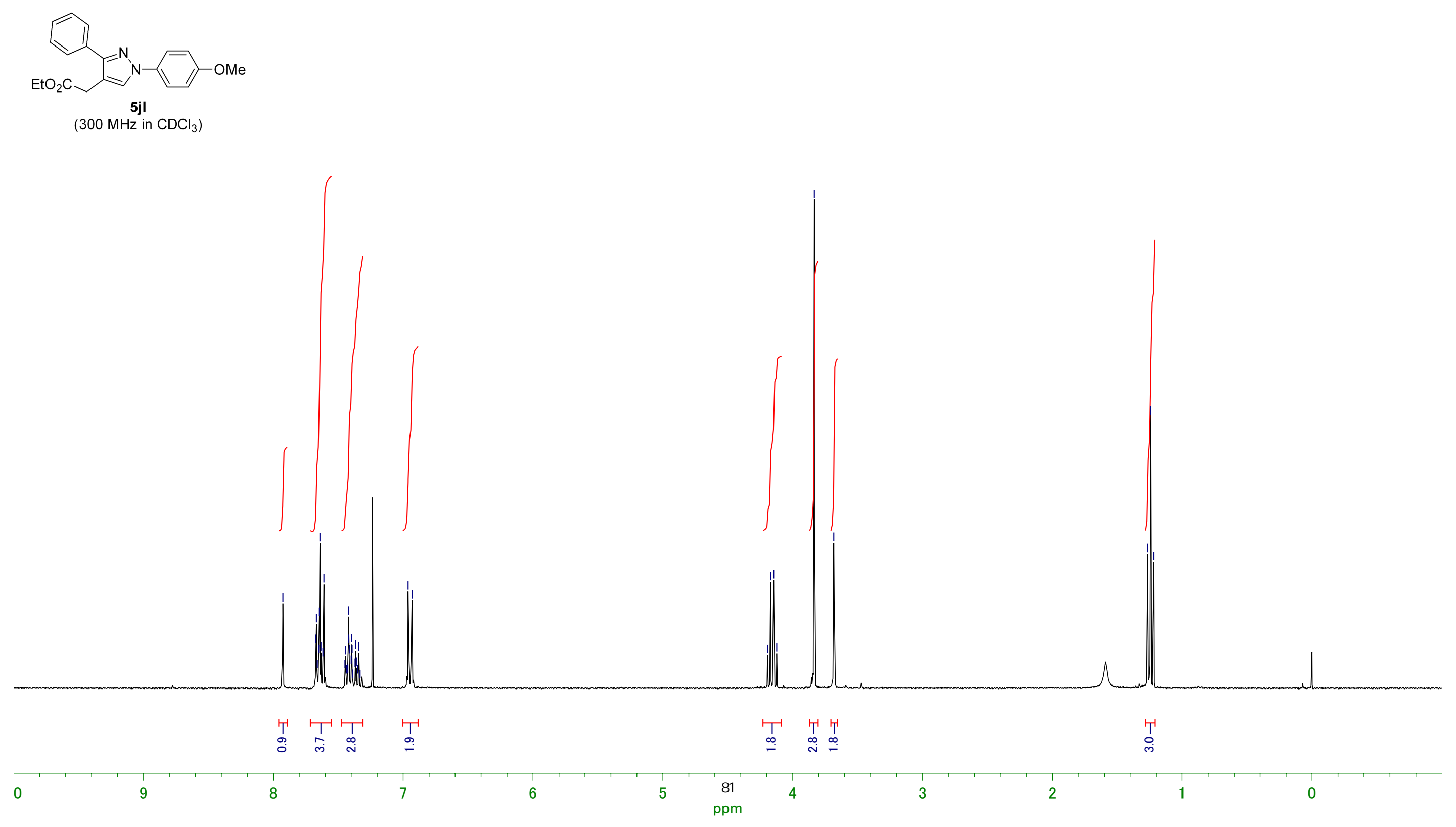

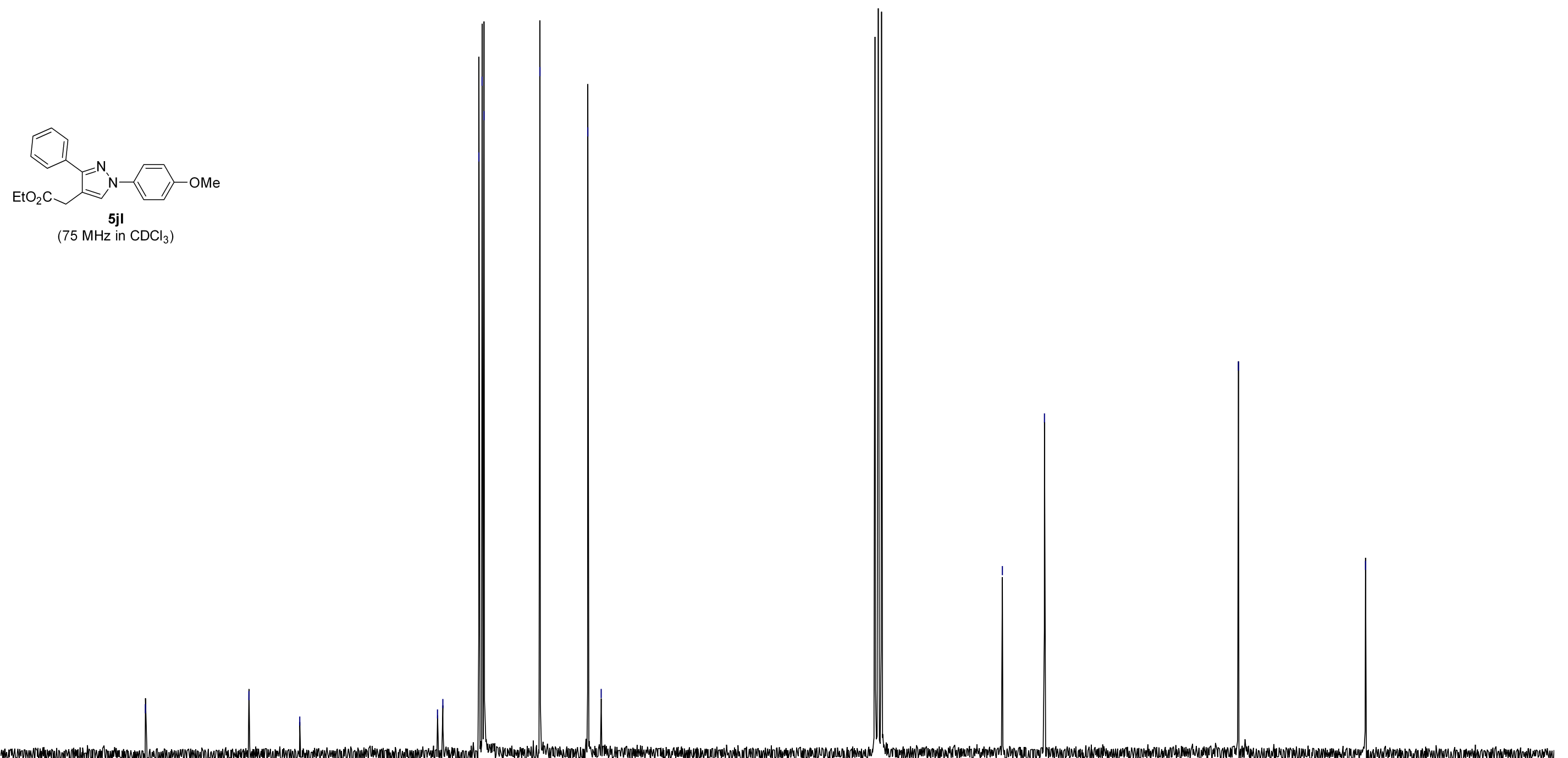

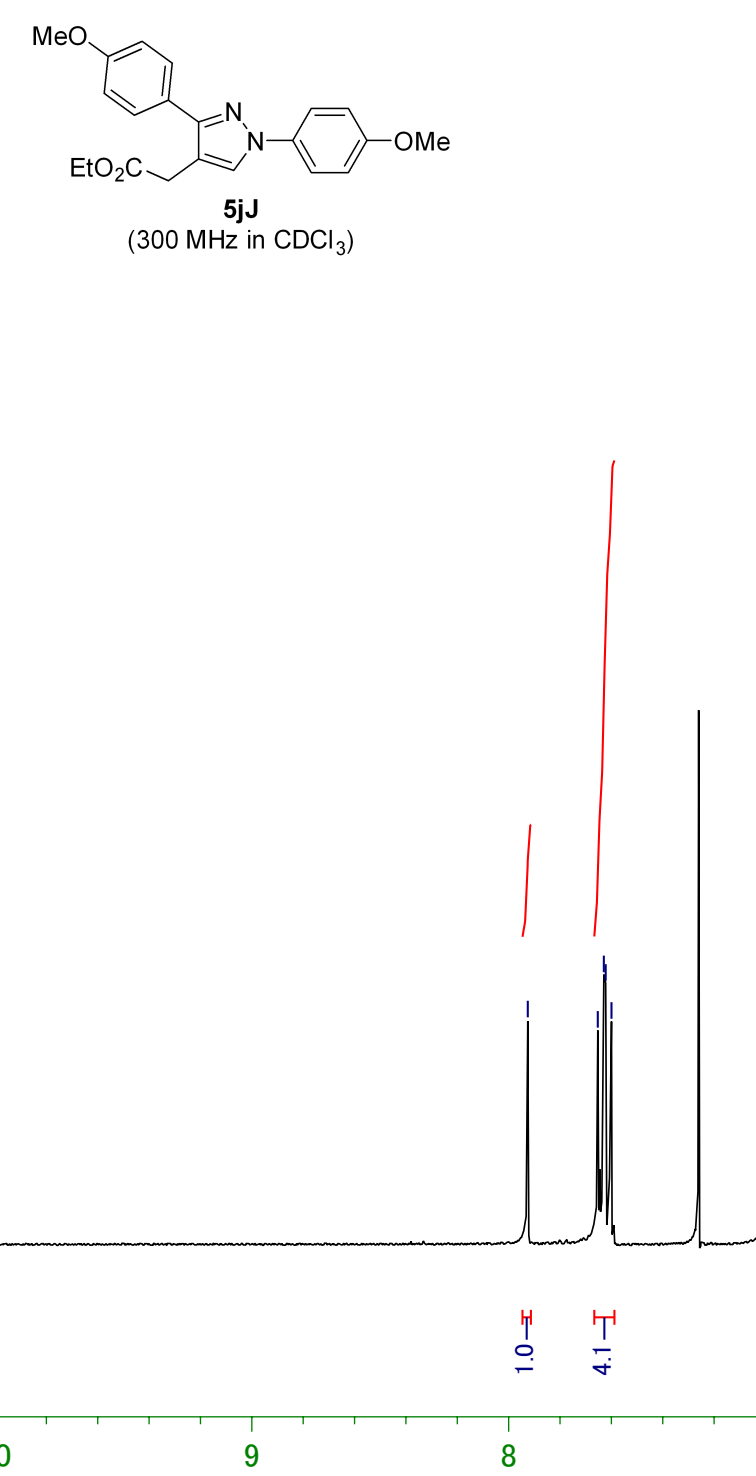

9

8

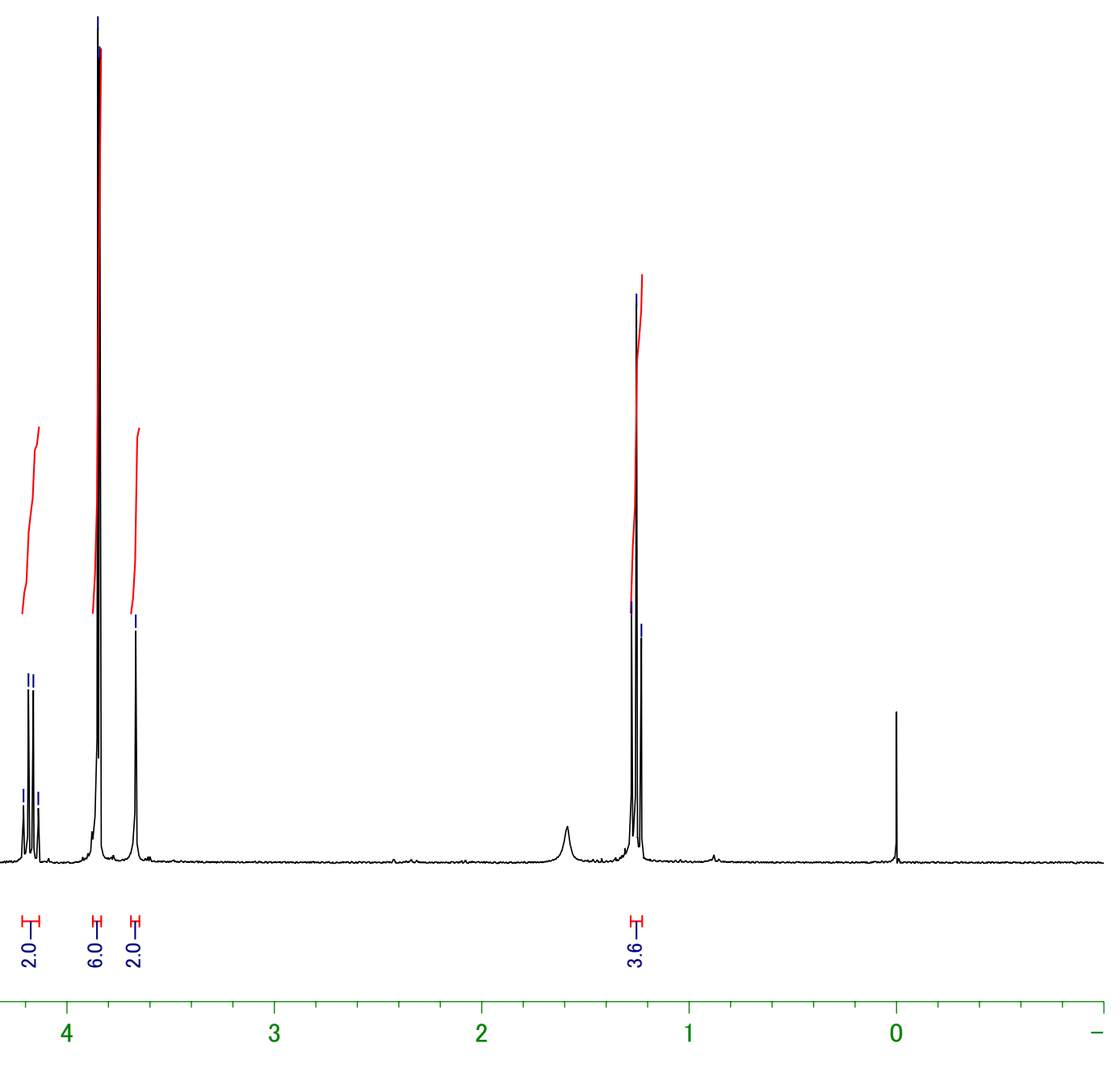




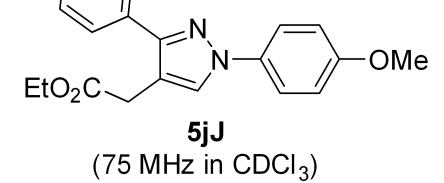

$75 \mathrm{MHz}$ in $\mathrm{CDCl}_{3}$ )

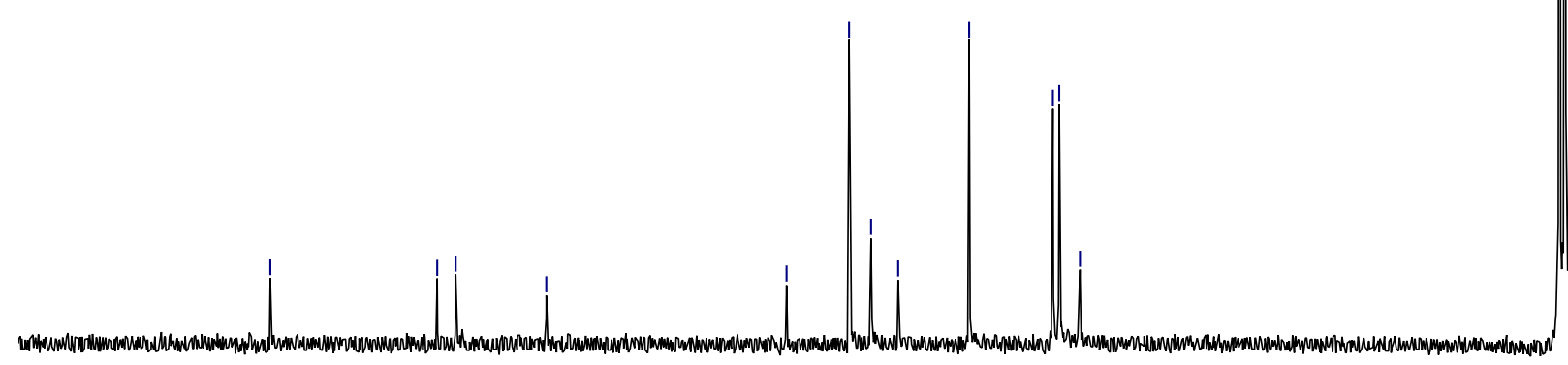



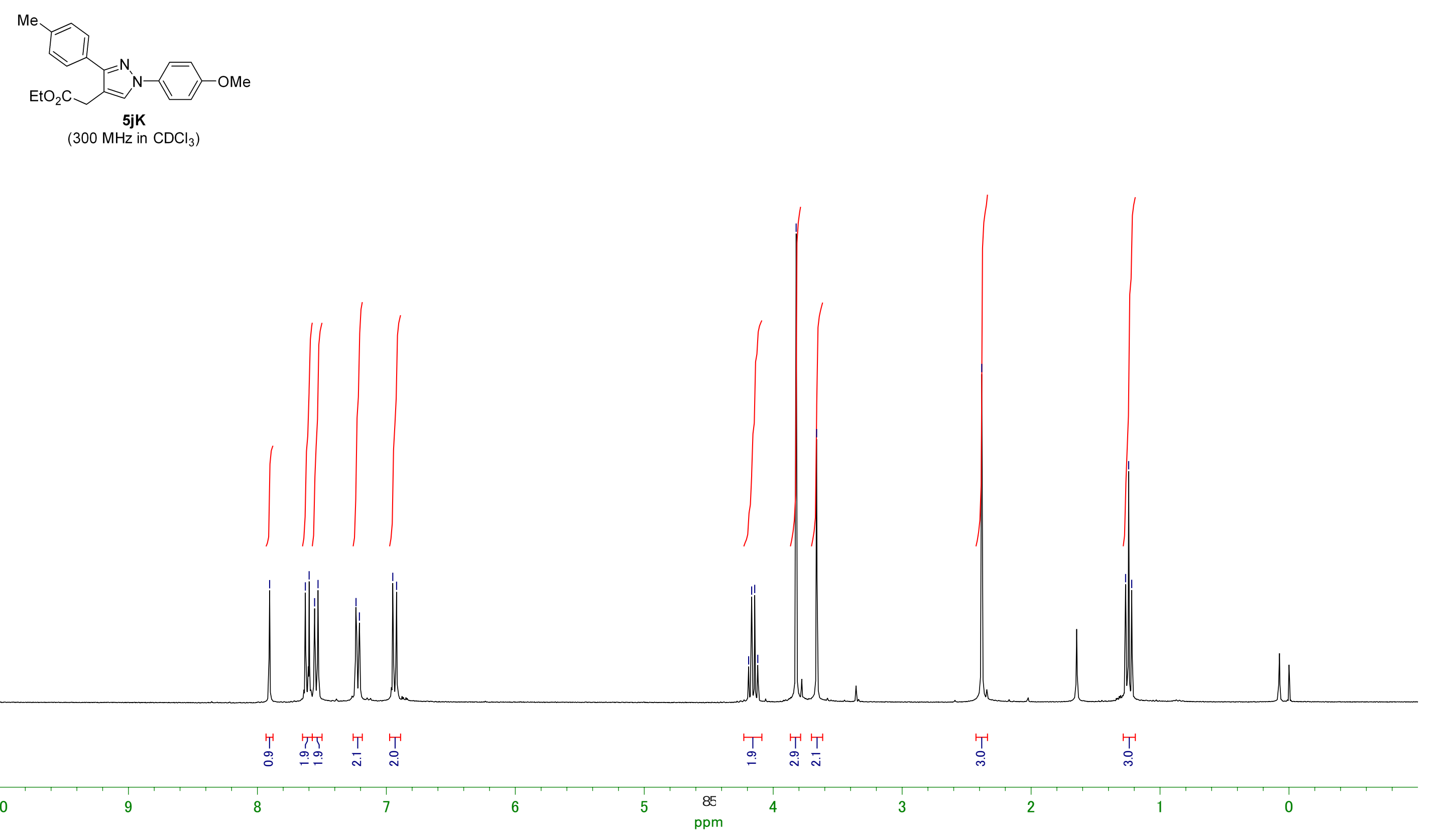


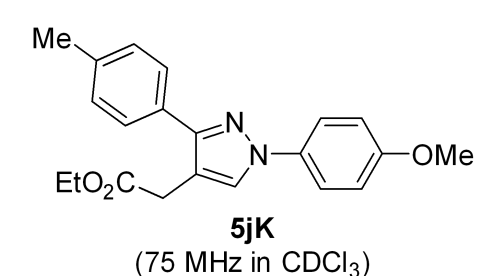

$\left(75 \mathrm{MHz}\right.$ in $\left.\mathrm{CDCl}_{3}\right)$ 


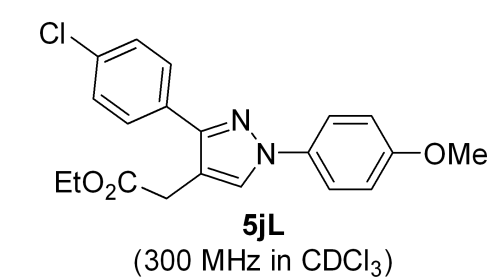

(300 MHz in $\mathrm{CDCl}_{3}$ )

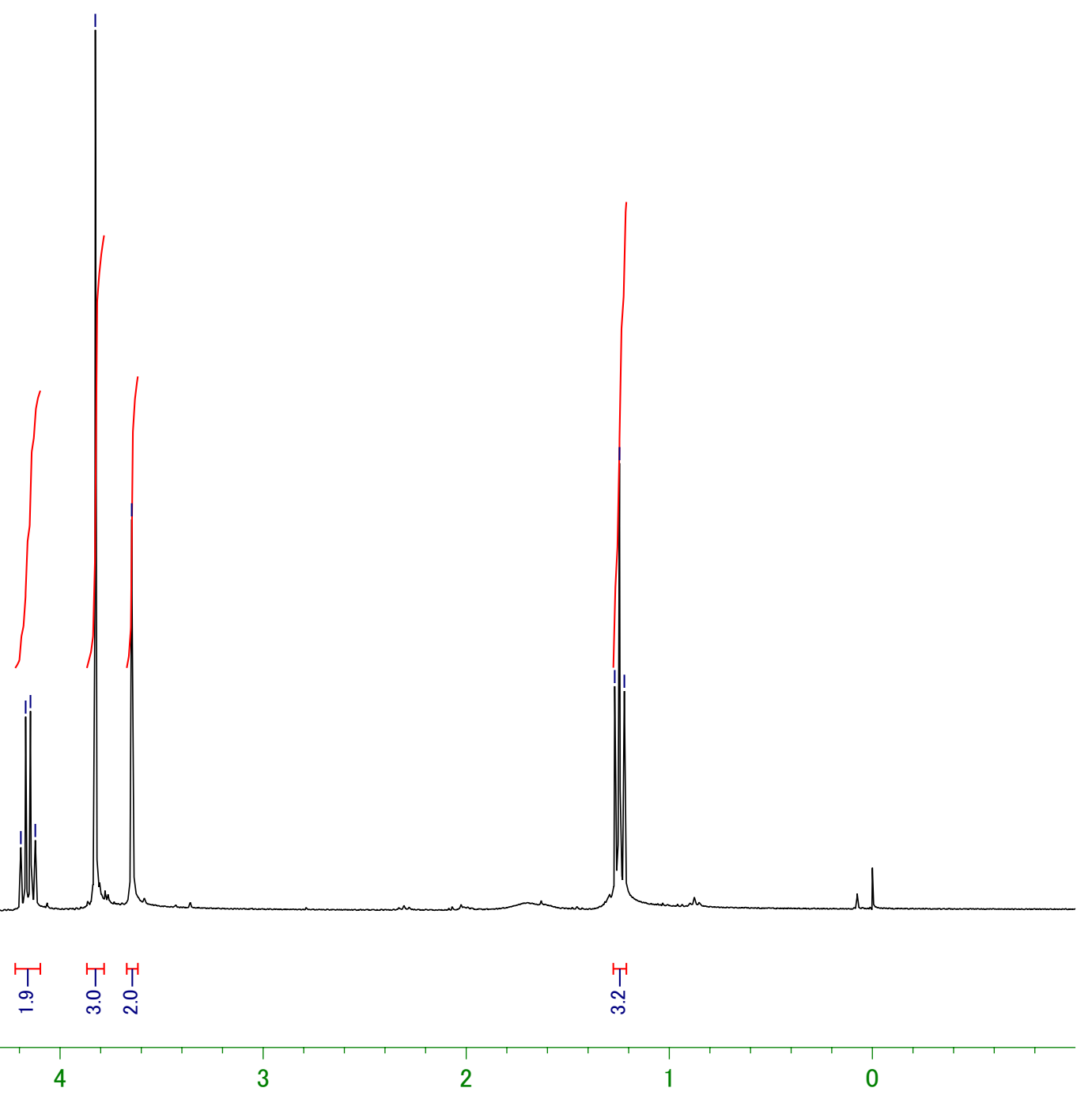




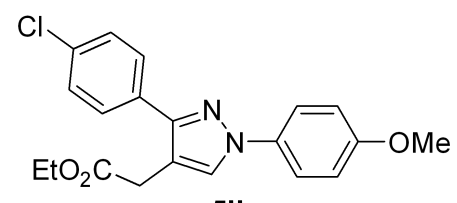

$\left(75 \mathrm{MHz}\right.$ in $\left.\mathrm{CDCl}_{3}\right)$

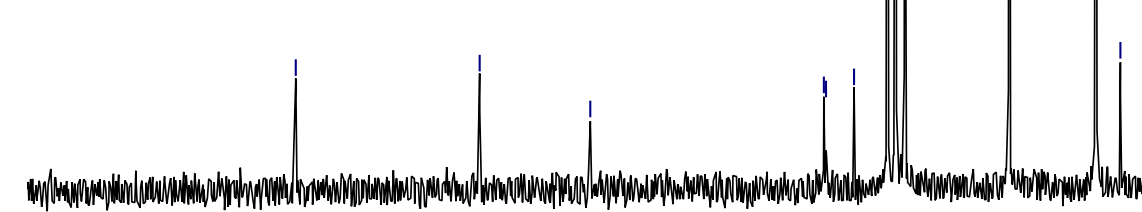

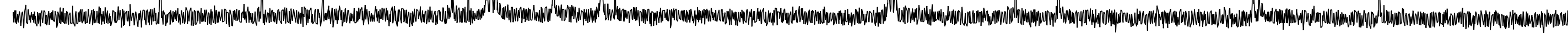




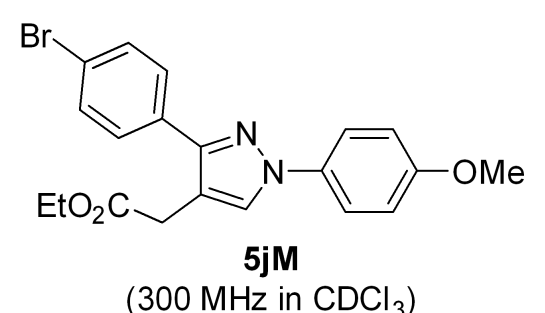

(300 $\mathrm{MHz}$ in $\mathrm{CDCl}_{3}$ )

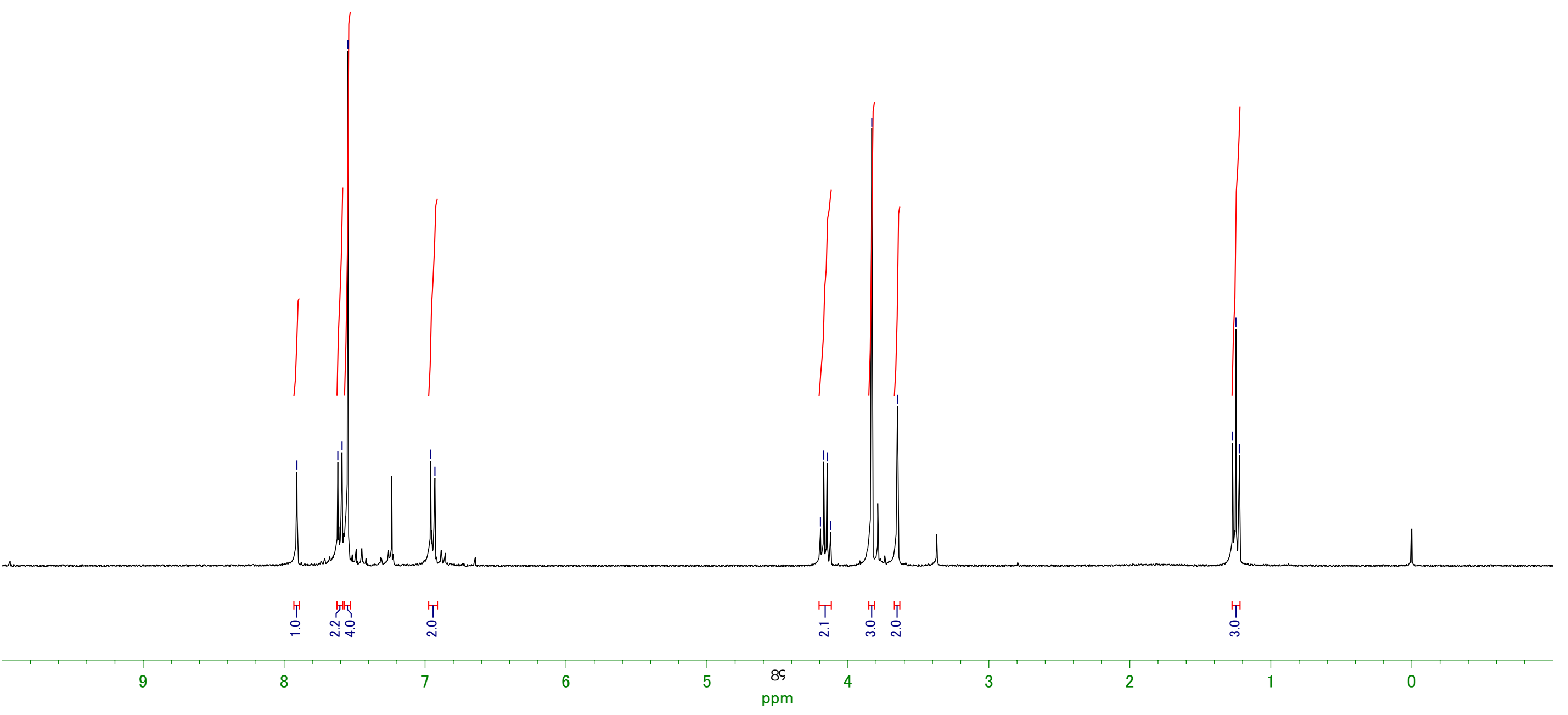




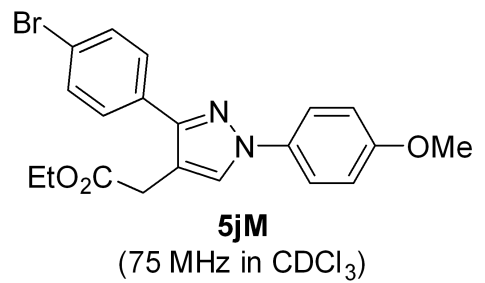

( $75 \mathrm{MHz}$ in $\mathrm{CDCl}_{3}$ )
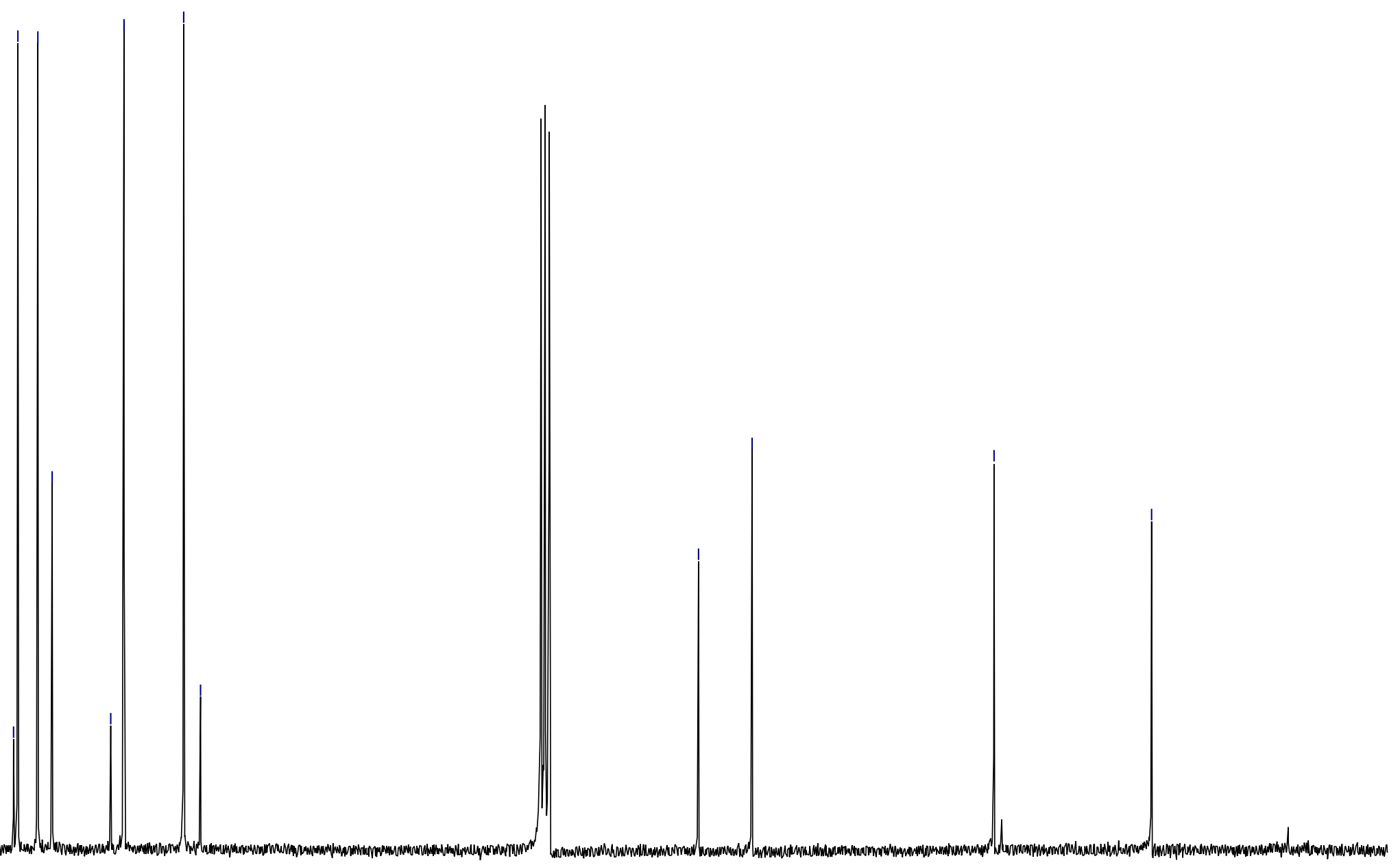


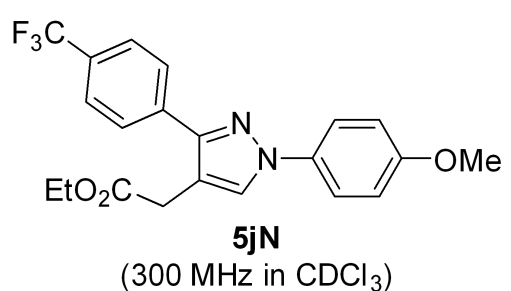

(300 $\mathrm{MHz}$ in $\mathrm{CDCl}_{3}$ )

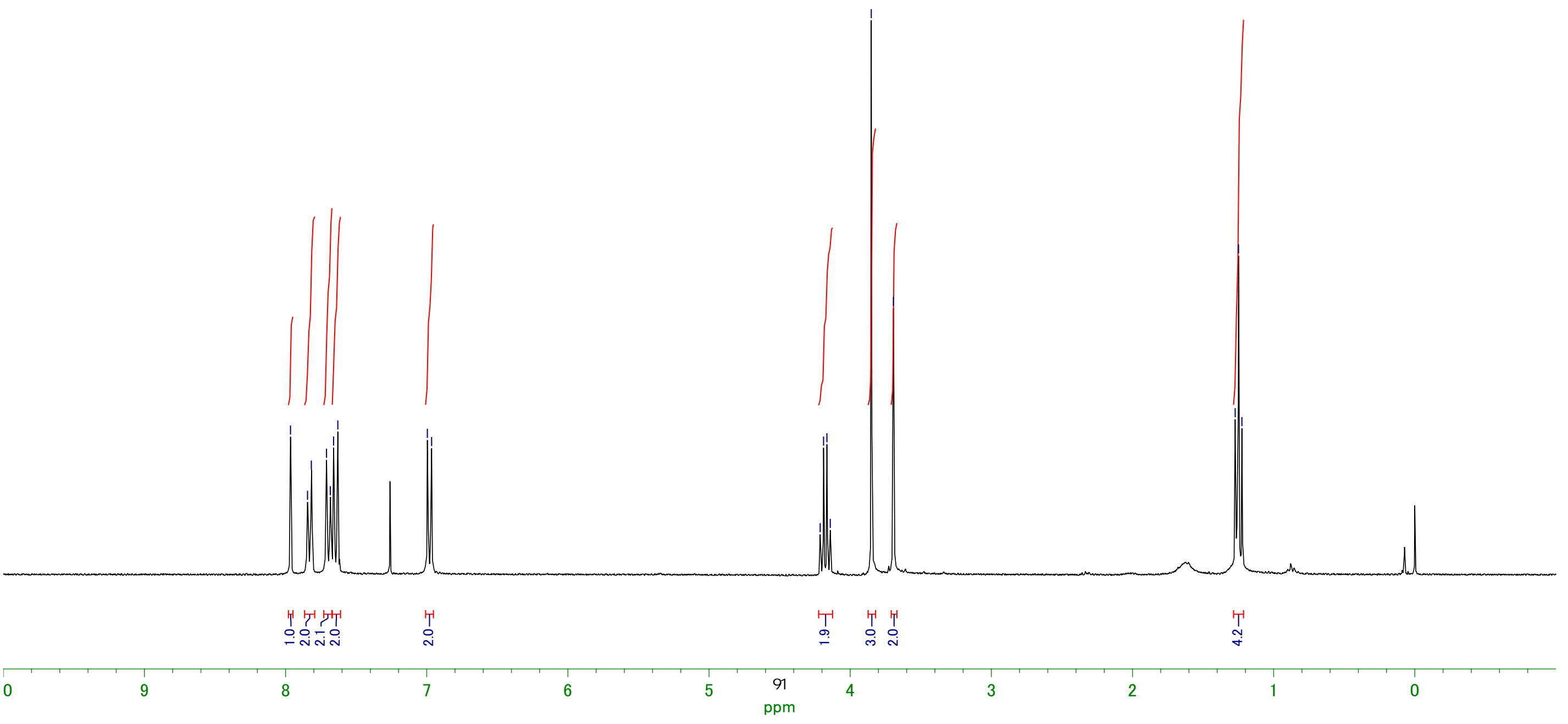




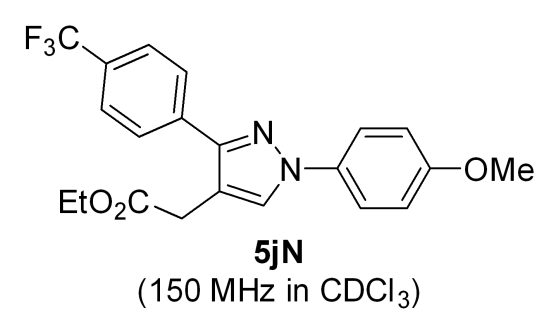




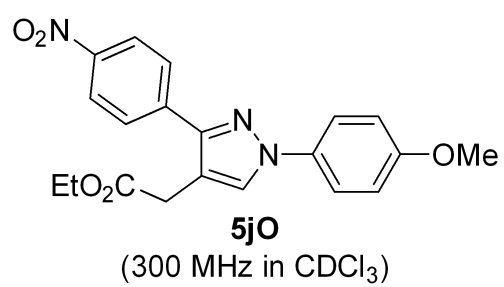

(300 MHz in $\mathrm{CDCl}_{3}$ )

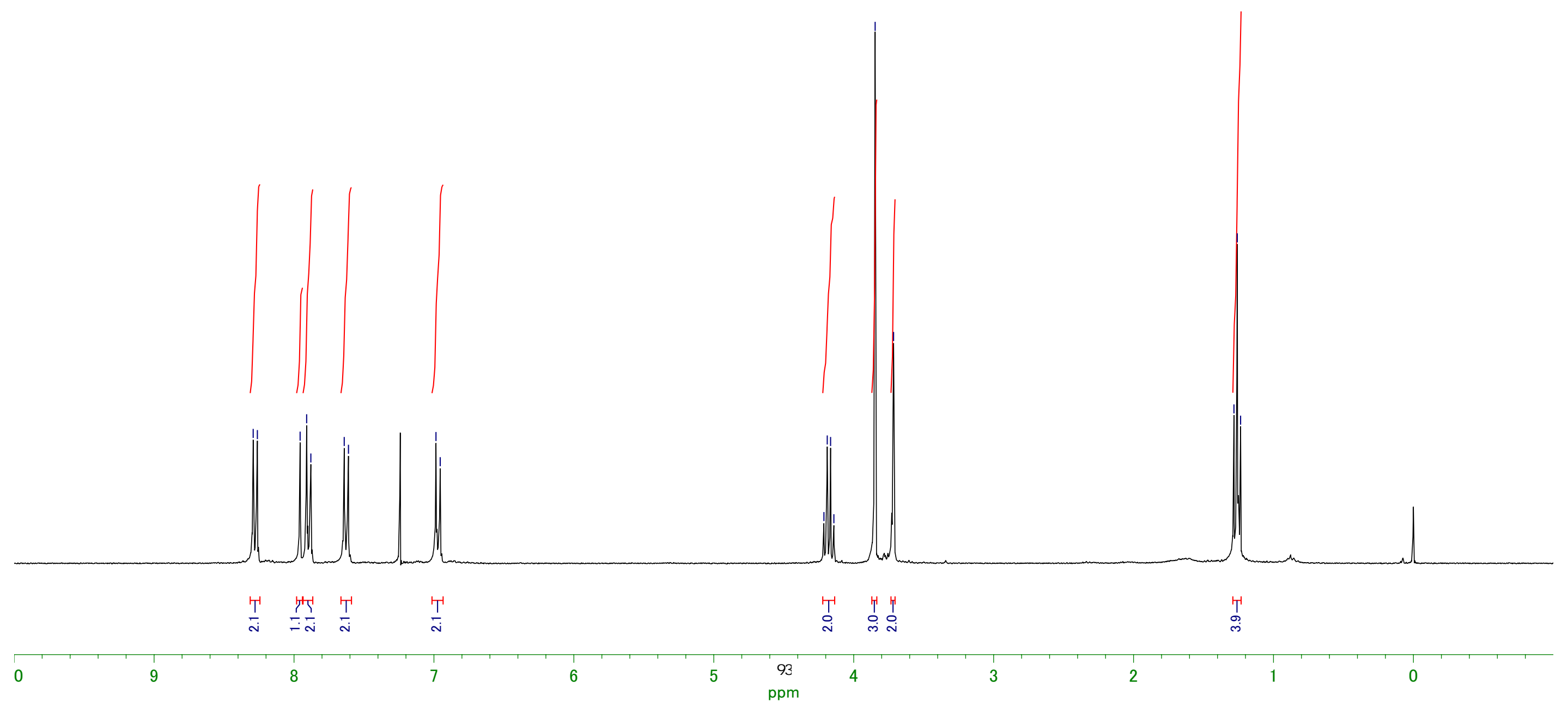



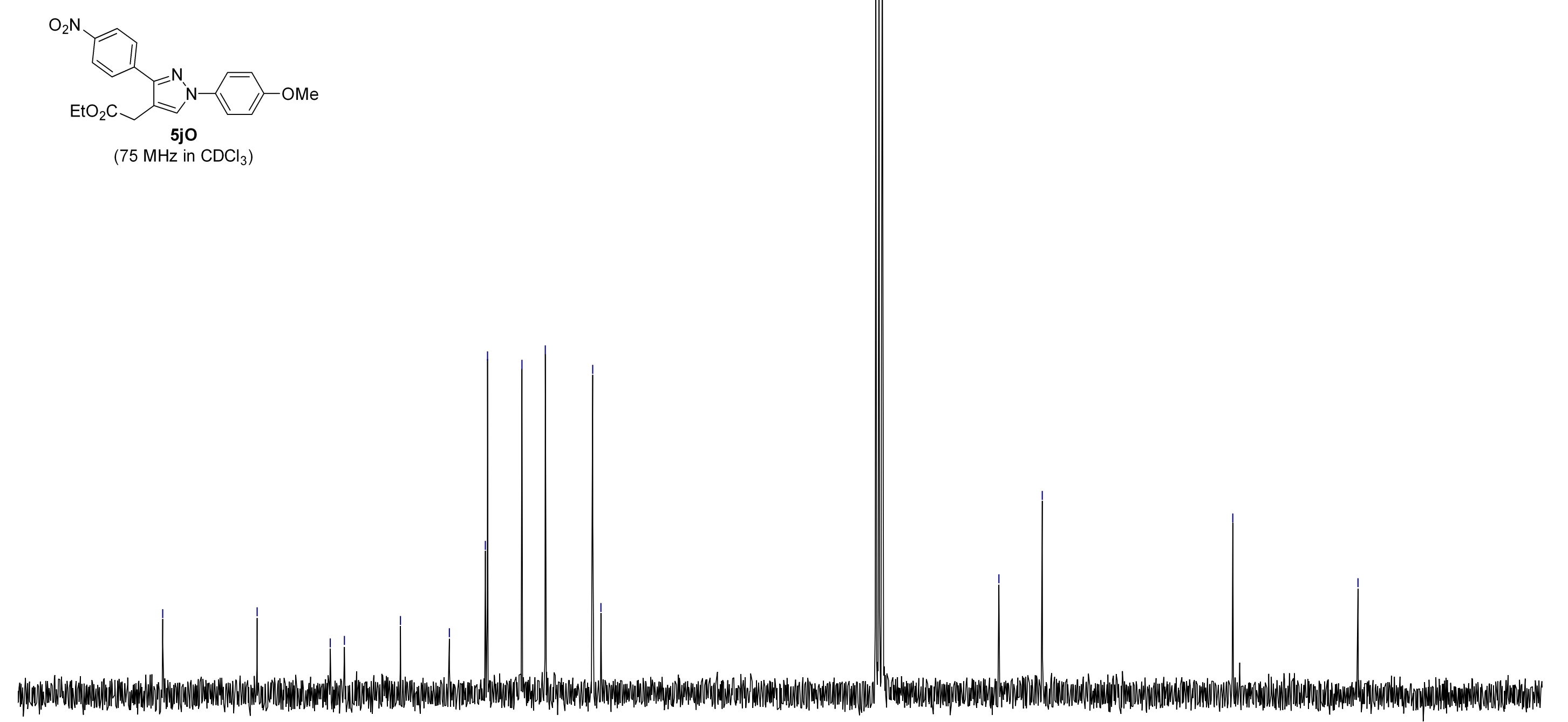

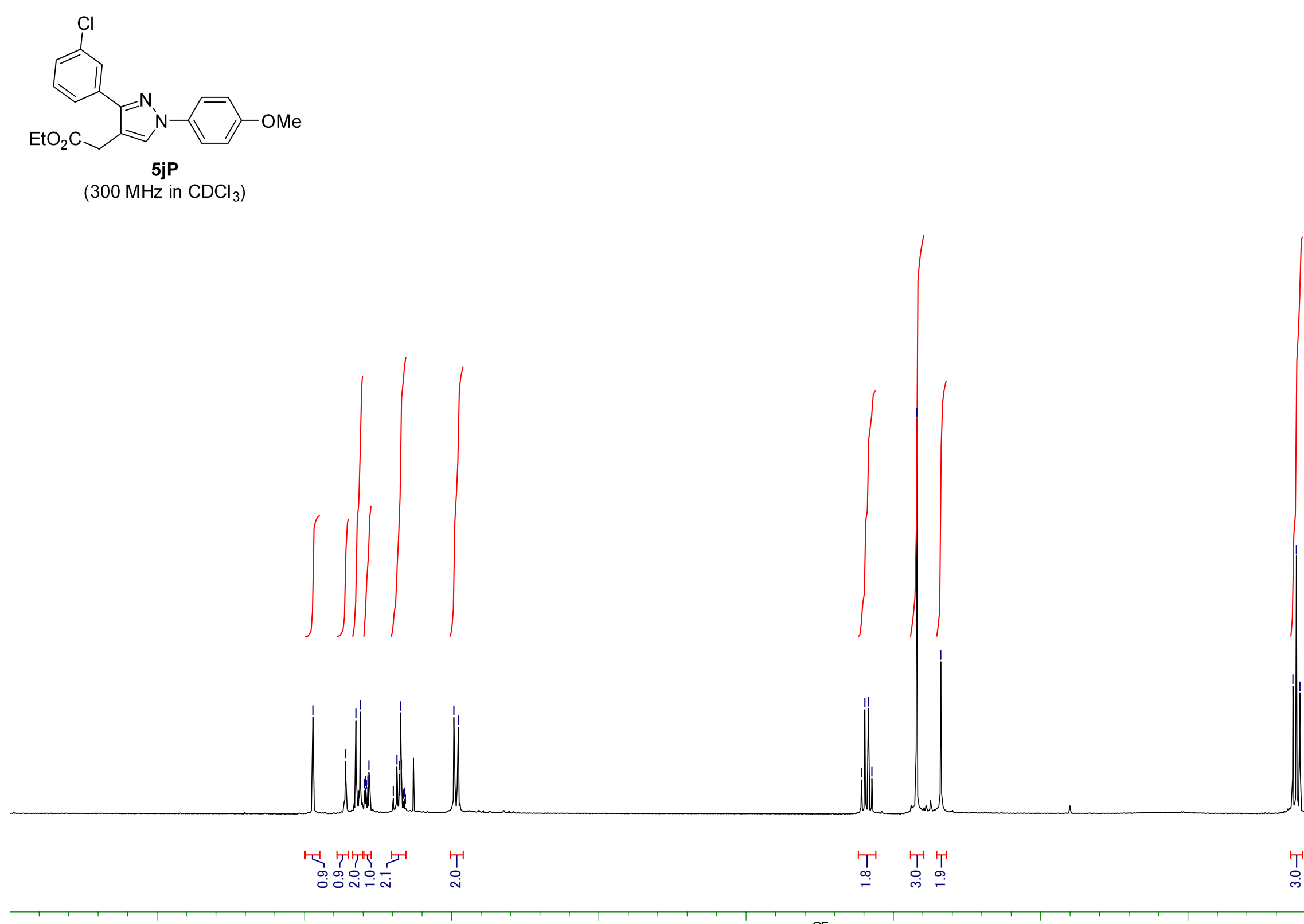

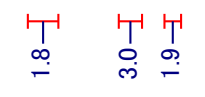




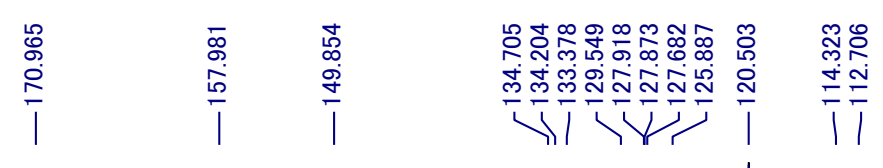

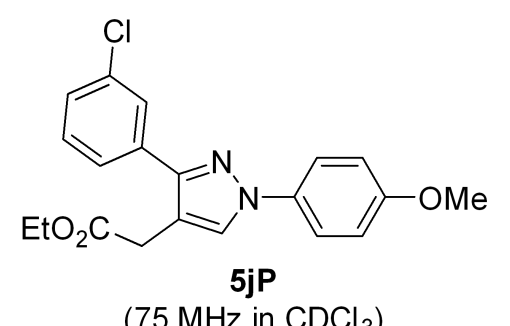

( $75 \mathrm{MHz}$ in $\mathrm{CDCl}_{3}$ )

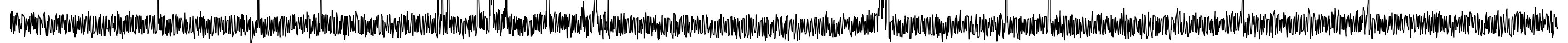



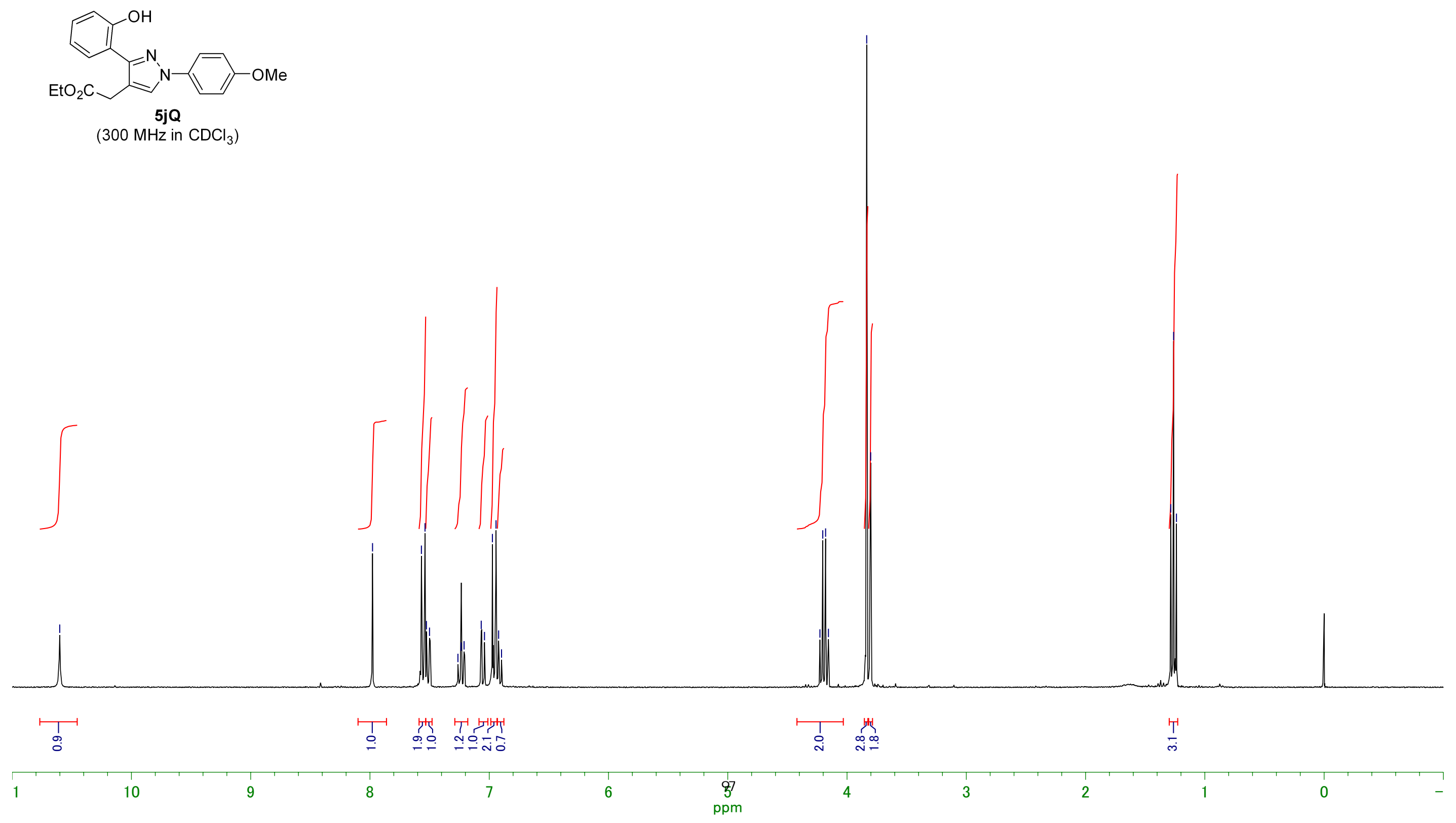


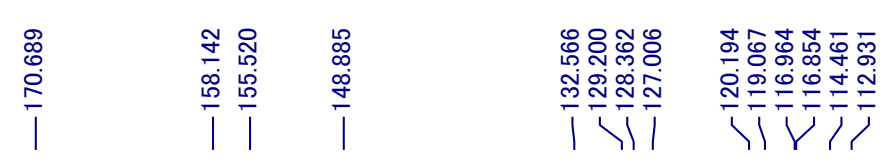

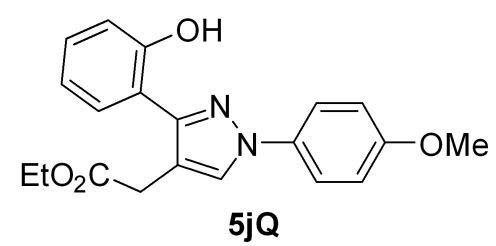

$\left(75 \mathrm{MHz}\right.$ in $\left.\mathrm{CDCl}_{3}\right)$ 

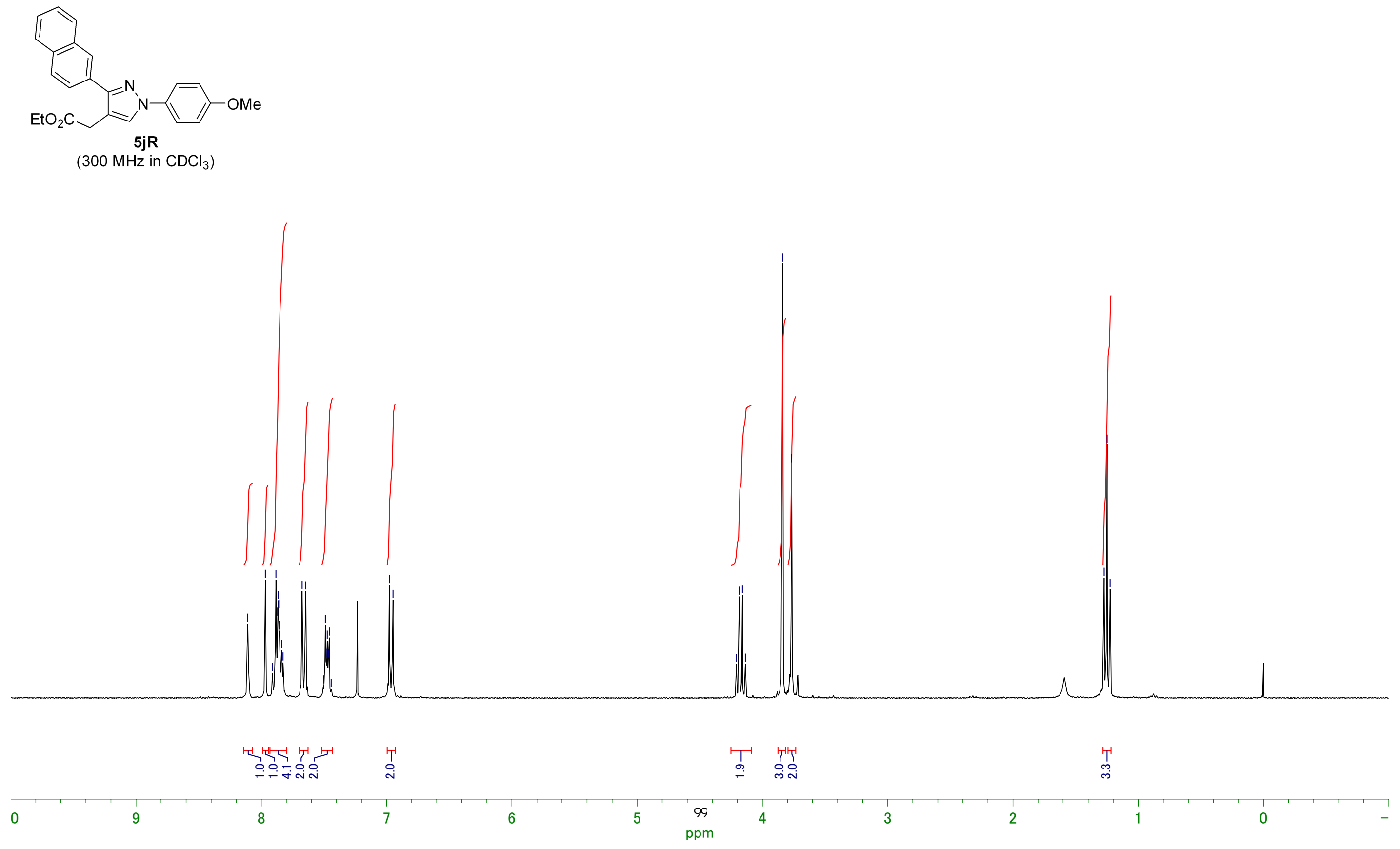

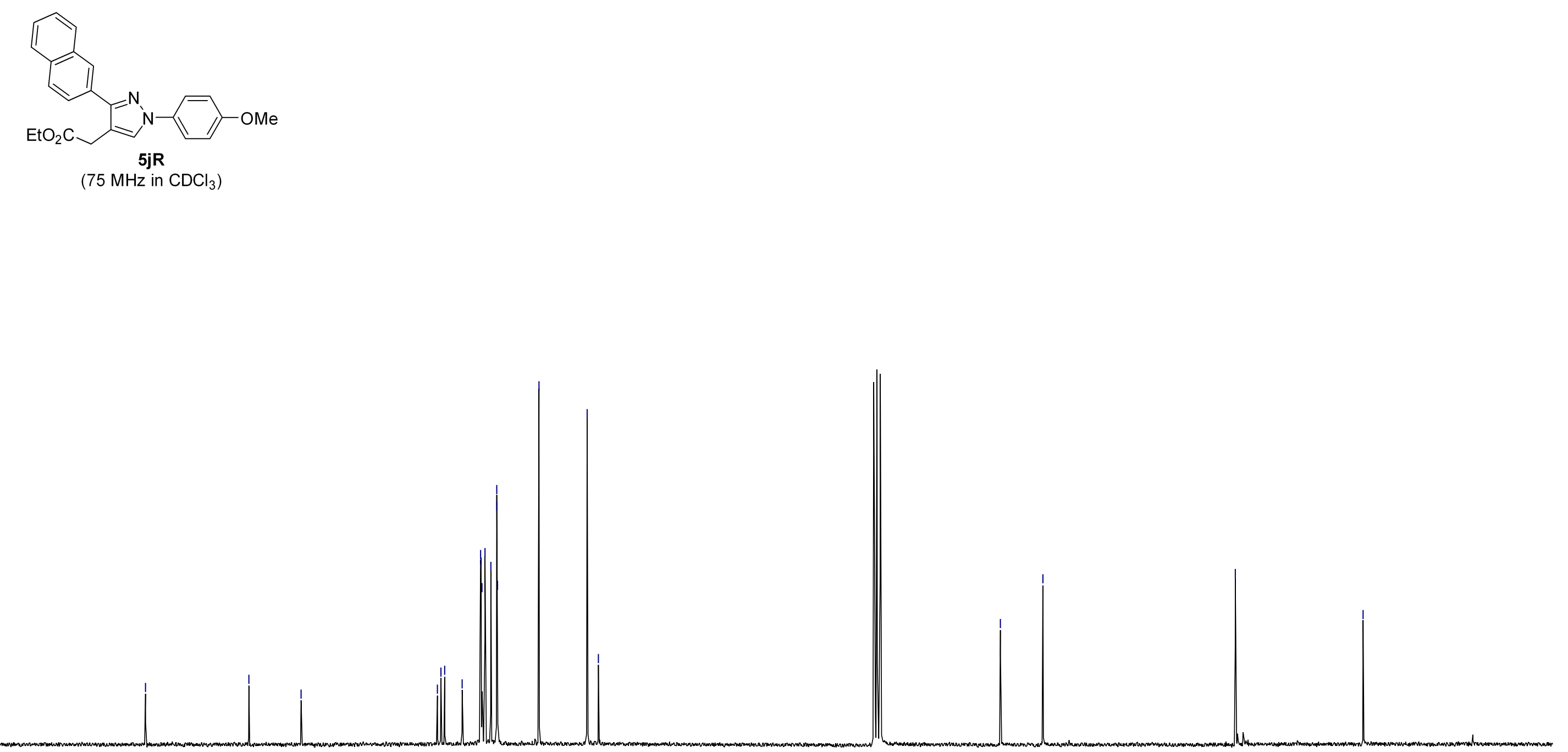


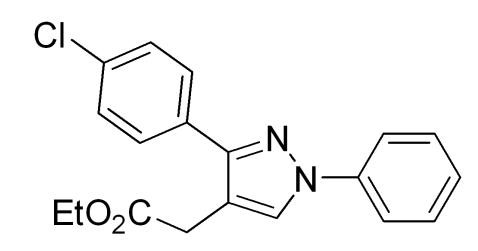

$5 \mathrm{jS}$

( $300 \mathrm{MHz}$ in $\mathrm{CDCl}_{3}$ )

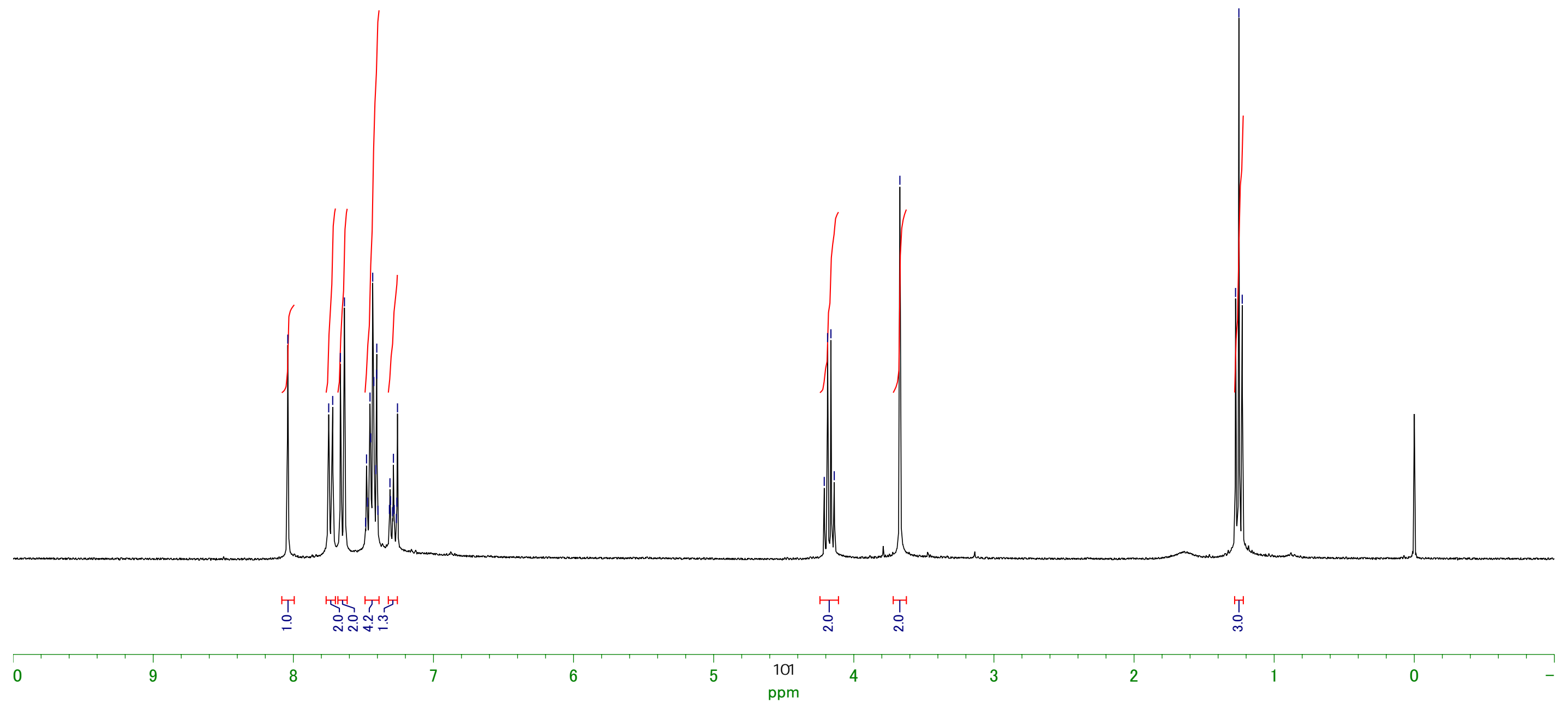




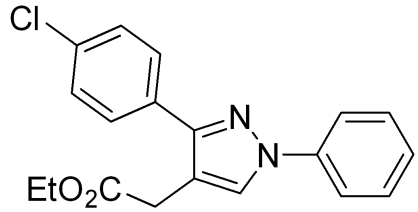

$5 \mathrm{jS}$

(75 $\mathrm{MHz}$ in $\mathrm{CDCl}_{3}$ )

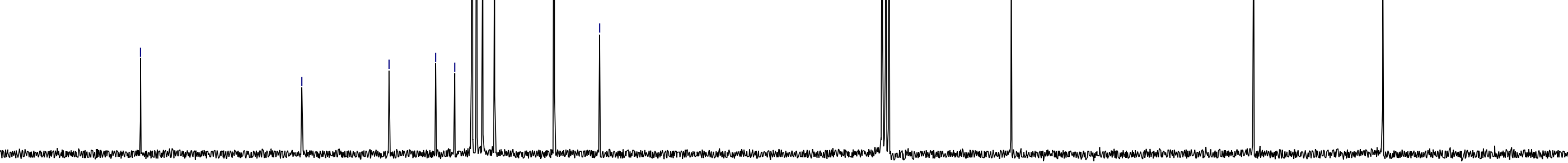



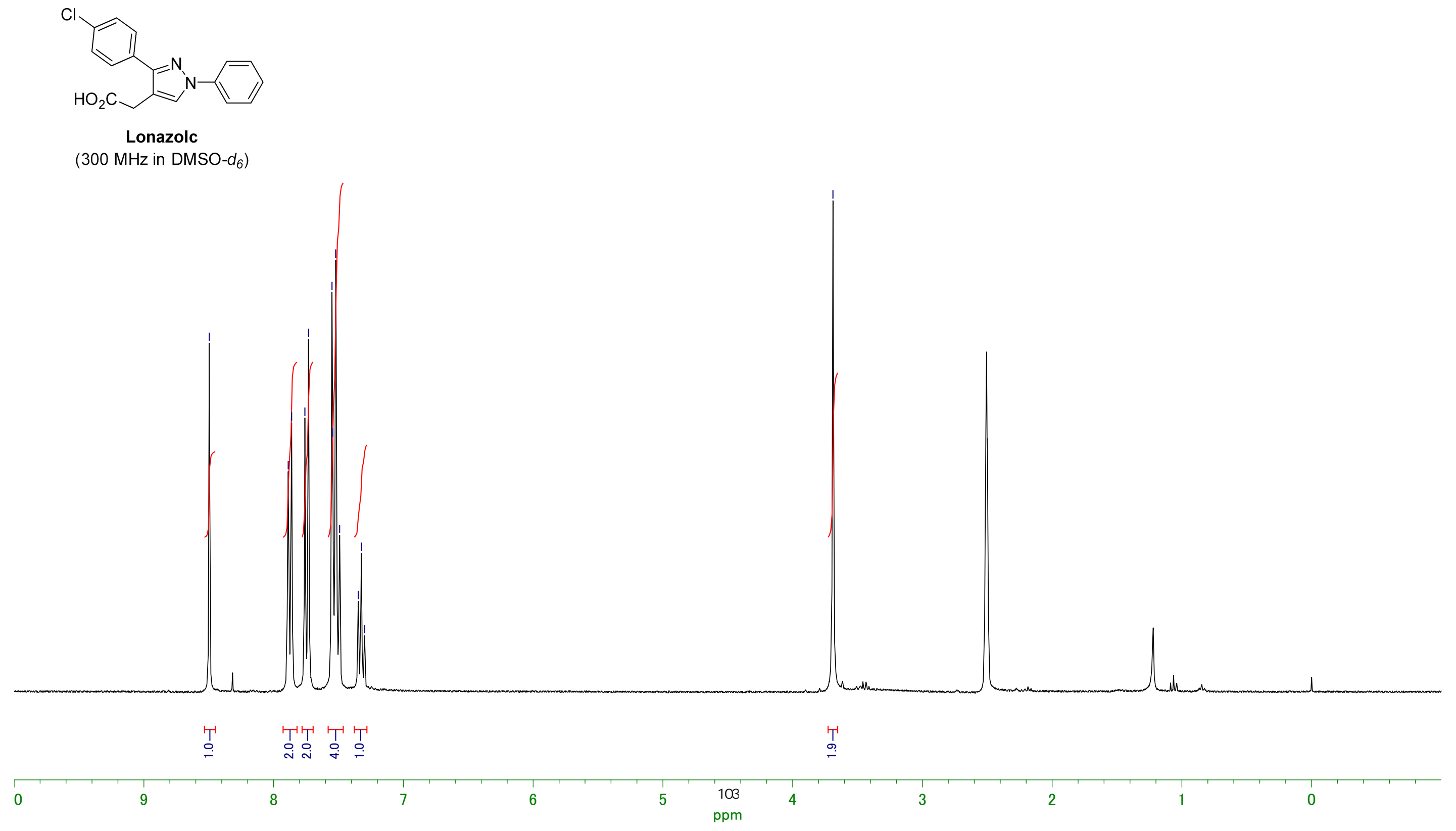


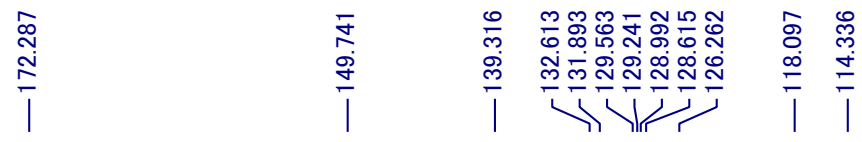

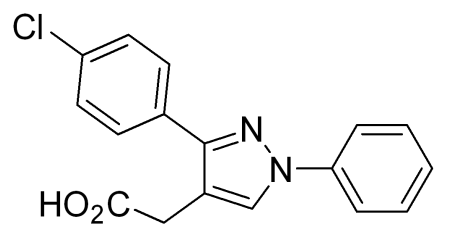

Lonazolc

(75 $\mathrm{MHz}$ in DMSO- $d_{6}$ )

$$
(75 \mathrm{MHz} \text { in DMSO-d } 6 \text { ) }
$$

